Rev. Adv. Mater. Sci. 56 (2018) 21-61

\title{
LENGTH SCALE PLASTICITY: A REVIEW FROM THE PERSPECTIVE OF DISLOCATION NUCLEATION
}

\author{
Mahdi Bagheripoor and Robert Klassen \\ Department of Mechanical and Materials Engineering, The University of Western Ontario, London, \\ ON N6A 5B9, Canada
}

Received: October 30, 2017

\begin{abstract}
Sub-micron and nano-size material systems and components are now regularly being fabricated for use in a wide variety of new applications. These systems exhibit mechanical properties that can be drastically different from their macroscopic counterparts and recently much work has focused on the size effects on the mechanical behaviour of materials. Although the size dependent behaviour has been observed in all of the crystal structures, the governing mechanisms have been found to be different. Different theories have been proposed to describe the size dependent behaviour of metallic samples and the governing mechanisms and it is well known that the surface plays an important role in the plasticity of small scales. Some of the theories indicate the importance of surface in nucleating dislocation and some other ones consider the surface importance as its effect on truncating dislocation loops and activation of internal sources. Moreover, recent studies have revealed that while dislocation based deformation in fcc metals is not very sensitive to temperature, deformation is strongly temperature dependent in bcc metals. The effect of orientation is more clear in the size scale behavior of hcp metals. This review covers recent literature that has focused on uniaxial compression of single crystals at the sub-micron and nanometer scale. The fundamental mechanisms governing the size dependent mechanical behaviour of different crystal structures are described. The effect of fabrication process and current experimental techniques for micro and nano-compression are studied as well.
\end{abstract}

\section{INTRODUCTION}

Nano-scale objects often exhibit properties that can be drastically different from their macroscopic counterparts, making them suitable for a wide variety of new applications, like micro/nanoelectromechanical systems (MEMS/NEMS) or advanced composite materials. Structural modifications usually occur due to surface relaxation or reconstruction, which both mechanisms resulting in surface energy minimization, while adding an extra contribution to surface stress [1]. As the sample dimension becomes small, the effect of nucleation at the surface becomes significant. Surfaces naturally introduce layers of atoms where their coordination number and the electronic structure are different from that of internal at- oms [2]. As a result, the physical and chemical properties of materials can be significantly different near the surface. The physical change results in a change in the surface energy, residual stress, and vacancy distribution as to facilitate the surface nucleation of defects $[2,3]$. The influence of the surface effect on the properties of the nano-scale sample is directly associated with the surface to volume ratio and obviously, the proportion of surface in the total volume of the sample will become higher when the physical dimension of the materials becomes smaller, resulting in increasing significance of the surface nucleation of defects.

Surfaces and interfaces can have a strong effect on the mechanical properties of nanostructures. In

Corresponding author: M. Bagheripoor, e-mail: mbagher6@uwo.ca 
the elastic regime, the Young's modulus can change compared to their bulk counterparts, due to the surface stress, and hence facilitate or hinder the deformation of the nanostructure, as a result of decreasing or increasing the elasticity coefficients. In the plastic regime, deformation mechanisms can be different in bulk materials and in nanostructures. For instance, the dislocation starvation process is one of the theories that has been proposed to explain the first stages of plastic deformation in nanostructures. Moreover, surface plays more important role in deformation of nanostructures since it acts as source of dislocation nucleation and surface diffusion could also affect the plasticity at nanoscales [4-6]. However, like bulk materials, boundaries between nanograins, are also affecting plasticity by emitting or absorbing dislocations or favoring the deformation by mechanisms like twinning $[7,8]$.

Recently, with the advances of nanotechnology and fabrication of nano-scale material there has been a great deal of interest in investigating the plastic deformation of nano-scale materials, such as nanograined, nanotwinned and nanostructured metals and a huge amount of research has gone into understanding dislocation-based plasticity at the nano-scale to achieve fundamental knowledge of plasticity of this scale, and provide design guidelines for reliable mechanical devices in the field of nano- or microelectromechanical systems [8-25]. By controlling the size of grains and producing nanocrystalline metals whit grains smaller than 100 $\mathrm{nm}$, strength levels can be easily exceed $4-5$ times higher than those with micron level grain sizes. Recent experiments have also shown that the high density of nano-scale growth twins in nanograined metals dramatically elevates the strength while providing considerable ductility $[9,10,23,26]$.

In bulk samples, the effect of microstructural scale on mechanical strength is understood either in terms of dislocation pile-up ideas or obstacles to dislocation migration [27]. However, these concepts cannot completely describe the behavior as the microstructural scales reaches dimensions as small as the average spacing between dislocations [28]. One of the major current focus in the nanomechanical community is the investigation of single crystalline strength at reduced dimensions through uniaxial deformation of micro or nano-sized samples [29]. At this scale and as metal sample sizes decrease to nano-scale, they are expected to contain few or no dislocations, and strength is commonly controlled by dislocations nucleated during plastic deformation which tend to escape the crystal by annihilating at a nearby free surface, a condition known as hardening by dislocation starvation [30-34]. Such ideas have been used to understand the deformation of nanometer-sized specimens [28].

Recent studies on the compression of single crystalline micro and nano-sized samples have indicated that the strength of crystalline metals, independent of sample size in bulk, increases dramatically as the sample size decreases to the nanoscale $[6,28,31,35-41]$. Size scale effects on strength and plasticity in nano-sized metals can be divided into geometry dependent and microstructure dependent effects [42] or as some scholars classified, extrinsic and intrinsic size effects. Different kinds of size effect have been reported in the last decade: external geometric size in single crystals, where the attained material strength inversely scales with sample dimensions, i.e. thin film thickness or nanopillar diameter due to defect interactions with free surfaces [43]. One such example is, Greer and Nix $[6,36]$ studies on electroplated Au micro/ nanopillars which resulted in conclusion that the overall sample dimensions artificially limit the length scale available for plastic processes and thereby significantly increase the material yield strength. According to Nix et al. [35], below a critical diameter, the traditional dislocation multiplication mechanisms that control work hardening stop operating and dislocations freely traverse the sample. In this dislocation starvation condition, the sample size is much smaller than a breeding constant for dislocation multiplication and all mobile dislocations will be annihilated at free surfaces before interacting with each other, so continuing plastic deformation is only possible if the applied stress is large enough to nucleate new dislocations [25,44]. This is also true when crystalline materials are indented at the nanometer scale. For this size indentation, the crystal volume probed is in fact so small as to be dislocation free. As a result the indentation curve is characterized by elastic loading followed by discrete displacement bursts (so called "pop-ins" [31,45-47]), associated with the dislocation nucleation [32]. Therefore, dislocation nucleation, either at nanomaterial surfaces or interiors, plays an important role in determining nanomaterial strength within the "Dislocation Starvation" regime [44]. However, the size effect is not similar for different structures and as an example while FIB-fabricated bcc Mo and $\mathrm{Nb}$ nanopillars also show size effects in their flow curves, the fundamental of this size dependent strength is quite the opposite and rather than annihilating at the free surfaces, dislocations in bcc metals have been found to form intricate networks 
as a result of mechanical deformation even in the smallest pillar size of $\sim 200 \mathrm{~nm}$ diameter $[48,49]$. Moreover, strength at the nano-scale depends not only on specimen size but also on the specimen and loading geometries. The importance of geometry was demonstrated in comparisons of indentation results of $\mathrm{Al}$ thin films and nanowires, where it was shown that nanowires are much weaker than thin films of the same minimum dimension [50].

Till now many research has been done on the size effect at different crystal structures. Strong size effect has been observed in face-centered cubic [35,51-54], body-centered cubic [48,55-58], hexagonal closed packed (hcp) [59-66], tetragonal [67-71] and rhombohedral [72] metals with a zero or small initial dislocation density that a smaller-is-stronger size effect operates, whereby smaller physical dimensions are accompanied by a pronounced increase in mechanical strength. About the fcc structure, the mechanism of deformation at small scale is well defined and investigated. However, about the bcc and hcp crystals, the active deformation mechanisms appear to be more complicated. Deformation in bulk bcc metals is governed by screw dislocation which its behavior and the effect of edge dislocation have been on the interest in small scales. Due to the limited slip systems in the hcp crystal structure compared to cubic metals, deformation may be accommodated by mechanical twinning, as well as by dislocation plasticity. Moreover, since the crystallographic texture greatly influences the shear stress necessary to activate slip, the effect of orientation in the deformation of bcc and hcp metals has been on the interest [73]. In this review, the main theories and proposed mechanisms for deformation of fcc, bcc and hcp single crystals under compression loading are studied and compared together. The main focus is on the sub-micron and nano-scales, since the plasticity at the micron-scale has been documented well $[43,74]$.

\section{NANO-COMPRESSION TEST}

There are many methods used to study the mechanical properties of materials. For the nanomechanical properties, the possibility of using all these methods is reduced because of either technical or monetary reasons. The methods that have been applied to study nano or small scale mechanics are as follows: nanoindentation [75], uniaxial compression [35], uniaxial tension [76], three/fourpoint pressing [77], the crack propagation [76], the plane-strain bulge test [78]. All these methods should presumably lead to the same conclusion about a certain property. However, there are some properties that cannot be studied by all of the mentioned methods. For example, the yield strength cannot be determined by nanoindentation. The physical property of the sample is another factor that limits the applicable tests, nanoindentation can not be applied on graphene since there is no thickness for graphene to be penetrated. Moreover, there is some inaccuracy with experimental condition. For example, nano-indantation with sharp tips introduces a nonuniform distribution of the mechanical strains or strain gradients within the contact area. The cost of the experiments is another factor that limits the available techniques for studying material properties at the nano-scale. For instance, uniaxial tensile test of nanopillars is very costly and requires more complicated procedure in sample fabrication and experimental testing [79].

In light of the above factors, the most common technique for exploring nano-scale mechanical properties with the fewest limitations and drawbacks especially that of the presence of strong strain gradients -is the uniaxial compressive loading of cylindrical nanopillars which was first introduced in 2004 by Uchic et al. [35] for compression of micro-sized pillars, and later extended to the nano-scale compression in 2005 by Greer et al. [36]. In these test, mimicking the bulk compression experiment, compression is applied using a diamond flat punch which could be controlled by a nanoindentation pendulum or an AFM tip, and the load displacement curve is simultaneously drawn as the pillar or particle compresses [28,79]. Experiments can be executed either under loading rate control or displacement rate control [28]. The most significant difference of this test than bulk compression test is that the nanocompression samples are not freestanding and they remain integrally attached to the bulk substrate to eliminate the need for nanomanipulation. In result, the substrate acts as the lower compression platen in this test [74].

\subsection{Experimental approaches}

Nanoindentation is the most general technique that has been used for studying nano-scale material behavior under compression, due to it's capability of applying force on a specific point with high control accuracy (loading rate control of micro-newtons per second and displacement rate control of nanometres per second). Nanoindentation, or instrumented indentation is a technique, appeared in the early 1980s and consists in measuring the force and displacement of a hard tip forced into the 


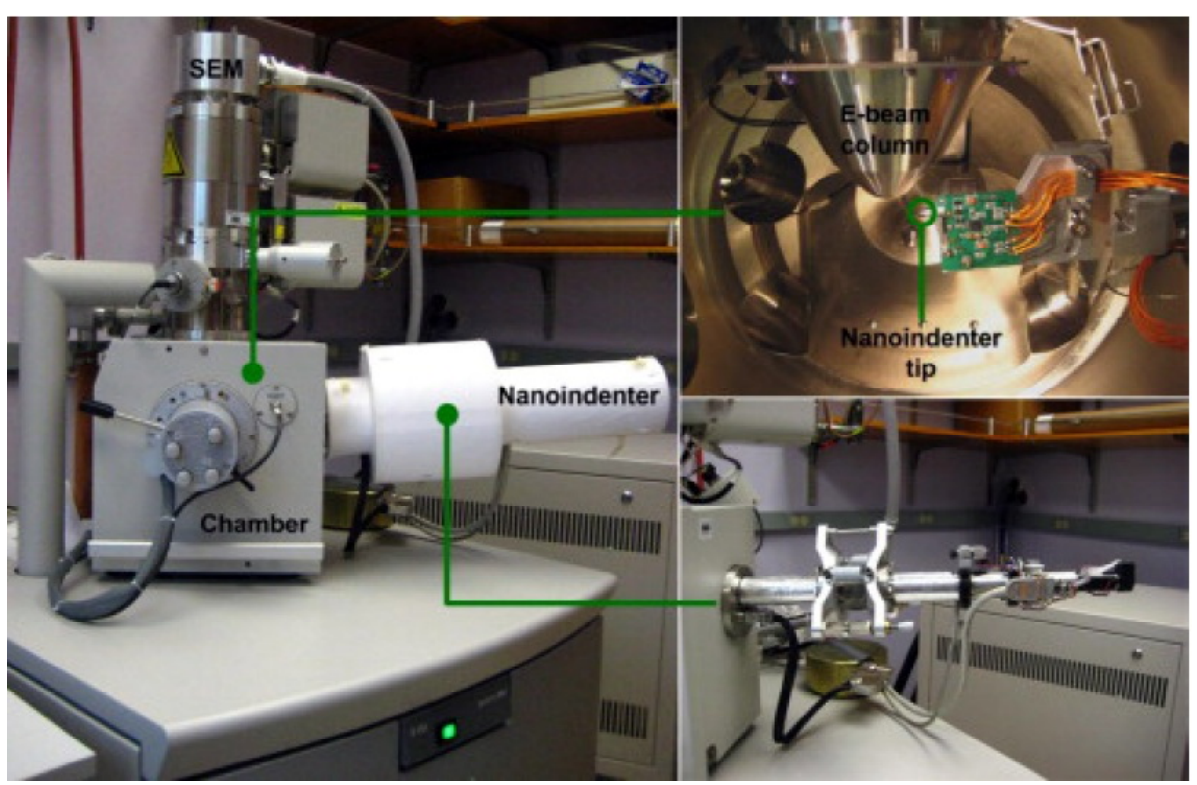

Fig. 1. Pictures of the SEMentor (SEM + Nano-indenter) and the inside of chamber. Reprinted with permission from Kim JY, Greer JR. 2009. Tensile and compressive behavior of gold and molybdenum single crystals at the nano-scale, Acta Materialia 57 (94), (c) 2009 Elsevier Ltd.

material. The load and displacement are measured through a change in capacitance between a reference and a movable electrostatic plate attached to the tip [80,81]. Oliver and Pharr [82], who have played a leading role in presenting models for quantitative nanoindentation, have presented a general overview on the development and recent advances of nanoindentation techniques [82]. In situ nanoindentation was reviewed in 2006 by Schuh [78], in which an excellent overview of in situ techniques with particular emphasis on technological advancements was provided. Nili et al. [83] traced developments and pinpoints significant recent advances, with emphasis on the applications of in situ nanoindentation techniques to materials systems, and highlighting the new insights gained from these in situ techniques.

Recently and with the advancement of technology, nanoindentation has been combined with different microscopic techniques to make it possible to observe material behavior during the deformation and investigate active mechanisms while the experiment is doing and in situ experimentation has gained significant attention due to its ability to enhance material characterization. Performing nanomechanical tests inside of a microscope device with real-time imaging, elucidate the specific deformation mechanisms that occur, as well as its occurrence time, and thus allows us a better understanding of the material's properties and overall performance $[83,84]$.
As Ghisleni et al. [85] pointed out, the main advantage of in situ SEM indentation techniques is the continuous observation of the surface deformation during the application of an external load on the specimen surface by an indenter. The large field of view, which helps to locate the feature of interest, and the sub-micron precision positioning allowed by the high magnification available through the SEM are the other advantages of doing nanoindentation in SEM instrument [85]. By performing the nanoindentation experiments in situ with a SEM instrument, one can observe how the specimen deforms under an applied load, which allows correlating the variation in the load-displacement curves to the variation in the specimen's topography. In particular, the influence of coating failure such as delamination, crack formation and propagation [8690], and surface shear band formation [90-93] can be correlated to a single discontinuity in the loaddisplacement data. Fig. 1 shows the Greer group's [94] in situ mechanical tester, SEMentor, in which the dynamic contact module (DCM) of the nanoindenter (Agilent Corp., Oak Ridge, TN, USA) inserted into a free port in the SEM (FEI Quanta 200, FEI Company, Hillsboro, OR, USA) and equipped with a custom-fabricated conductive diamond tension/compression grips. Instrumentation companies like Hysitron, has provided Picolndenters that could be installed on the SEM and used for in situ mechanical testing. However, even though in situ SEM indentation can observe the deformation 


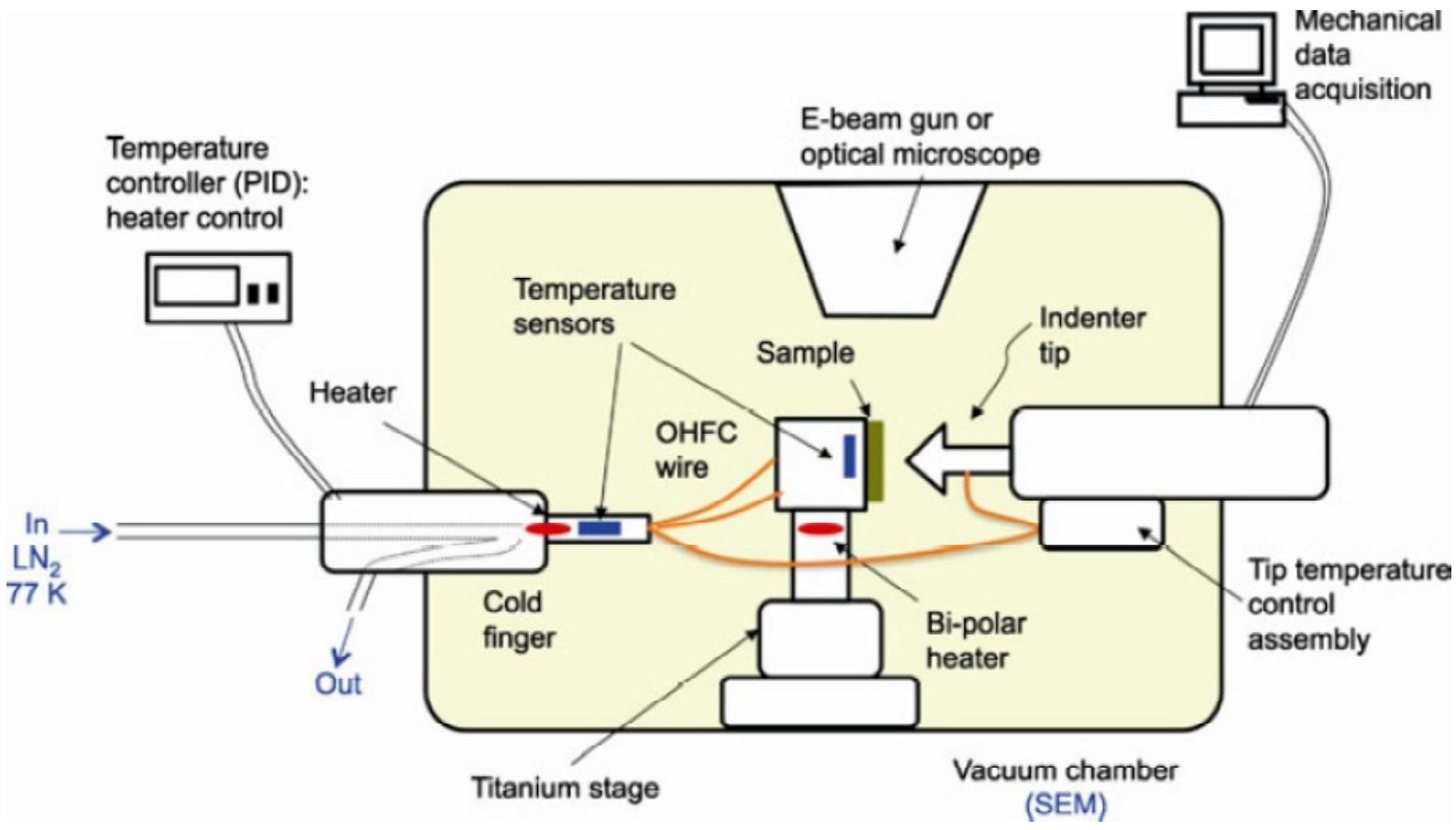

Fig. 2. Schematic diagram of an in-situ nanome-chanical tester integrated with the cryogenic system. Reprinted with permission from Lee SW, Cheng YT, Ryu I, Greer JR. 2014. Cold-temperature deformation of nano-sized tungsten and niobium as revealed by in-situ nano-mechanical experiments, Science ChinaTechnological Sciences 57: (c) 2014 Springer Nature.

on the surface of the indented specimen, some physical phenomena that might take place underneath the surface such as phase transformation, or nucleation and propagation of dislocations are hardly visible by SEM observation.

Lee et al. [95,96], developed an in-situ cryogenic nanomechanical testing system by combining the nanomehcanical tester (InSEMTM, Nanomechanic, inc), SEM (Quanta, FEI), and cryogenic systems (Janis Research Company, LLC), to study smallscale mechanical behavior of materials at low temperatures. Fig. 2 shows the schematic diagram of cryogenic system consisting cold finger, Si-diode temperature sensors, joule heaters, radiation shielded oxygen-free high thermal conductivity copper (OFHC) lines, vacuum shielded coolant transfer line, the liquid nitrogen dewar, and temperature controller [95].

Following early load sensor integration developments, in situ TEM mechanical testing with quantitative load measurement including mechanical probing modes such as nanoindentation expanded toward commercialization in the last decade [97]. These developments are particularly useful for conducting size effects experiments, whereby quantitative in situ TEM mechanical testing is an ideal tool for studying the origin and principles of the material deformation through dislocation and twin-

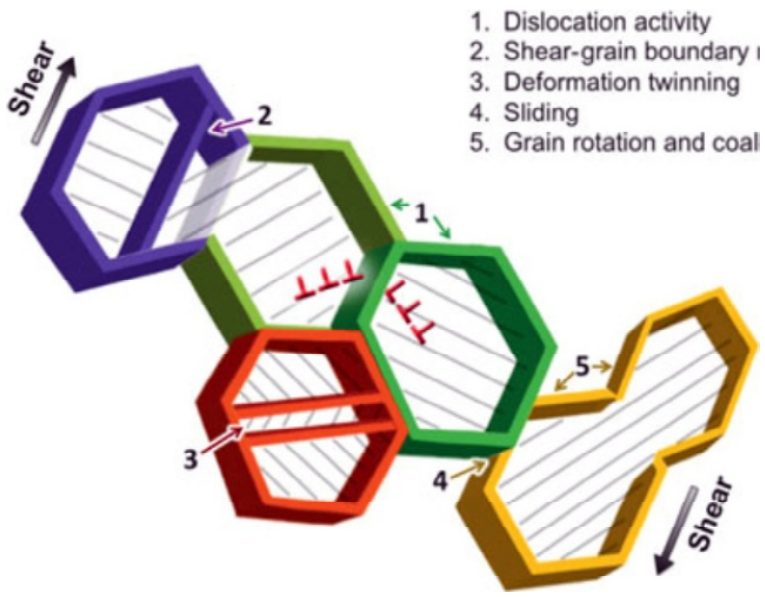

Fig. 3. Schematic of various deformation mechanisms that can be studied by in situ TEM mechanical testing. Reprinted with permission from $\mathrm{Yu} Q$, Legros M, Minor AM. 2015. In situ TEM nanomechanics. Mrs Bulletin 40, [97]: (c) 2015 Cambridge University Press.

ing observation. Fig. 3 shows some the of involved deformation mechanisms in size effects which can be studied by in situ TEM.

Various approaches of mechanical testing inside a TEM exist today. They span from using TEM holders with a simple mechanical actuation to elaborated testing units that fit inside the pole pieces. From 2003 to 2005, Hysitron ${ }^{\circledR}$ and Nanofactory In- 

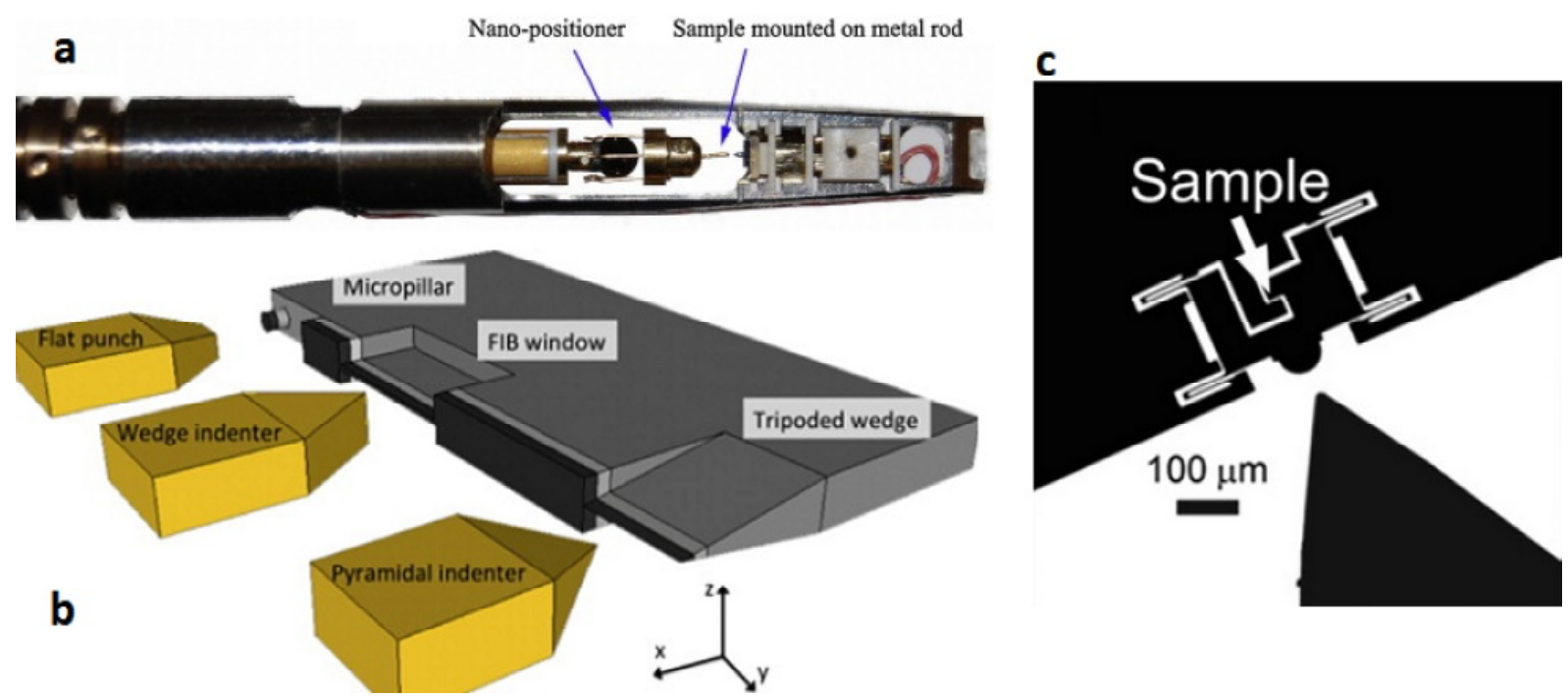

Fig. 4. Examples of in situ TEM mechanical testing techniques: (a) Tip of a classical straining holder (b) sketch of the various types of straining configurations used in nanoindentation holders, (c) Push to pull device with a Hysitron PI-95 nanoindenter. Reprinted with permission from: Legros M. 2014. In situ mechanical TEM: Seeing and measuring under stress with electrons. Comptes Rendus Physique 15, [98]: (c) Elsevier Ltd. Carlton CE, Ferreira PJ. 2012. In situ TEM nanoindentation of nanoparticles. Micron 43 [105]: (c) Elsevier Ltd. Kacher J, Yu Q, Chisholm C, Gammer C, Minor AM. 2016. In Situ TEM Nanomechanical Testing. In: Prorok B., Starman L. (eds) MEMS and Nanotechnology, Volume 5. Conference Proceedings of the Society for Experimental Mechanics Series [106]: (c) 2014 Springer Nature.

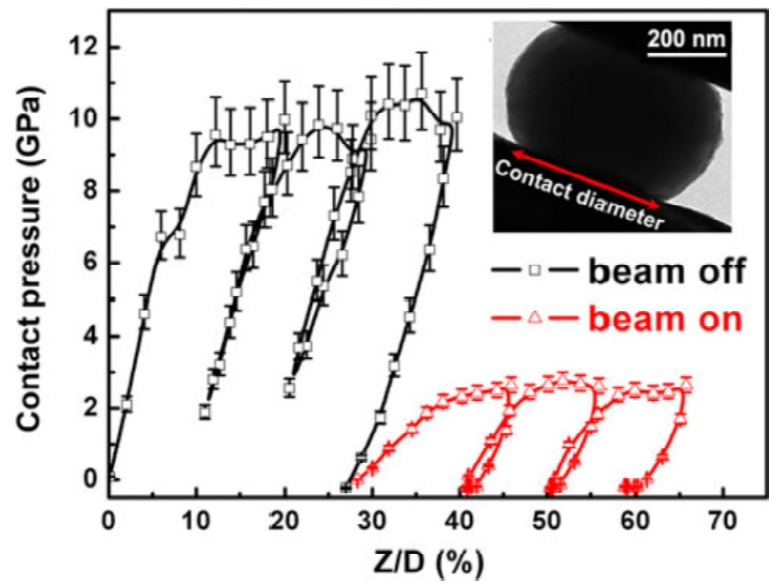

Fig. 5. The effect of E-beam on the mechanical behavior of an amorphous silica particle. The contact pressure decreased about four times when electron beam was switched from off (black curve) to on (red curve). The inset is a frame from the recorded movie during compression test. Reprinted with pesrmission from Shan ZW. 2012. In Situ TEM Investigation of the Mechanical Behavior of Micronanoscaled Metal Pillars. Jom 64, [105]: (c) 2014 Springer Nature.

struments $A B^{\circledR}$ companies further implemented the indenting holders by adjoining them a micro load cell [98].

In the recent years, nanoindentation, AFM, MEMS-based testing device and scanning tunneling microscope [99] probe were integrated with the TEM platform $[97,98,100]$. In situ mechanical testing via nanoindentation, AFM and MEMS device have revealed novel deformation phenomena and size dependent responses in nano-sized materials [28,59,101-104]. Different holder setups have been developed based on the desired mechanical test and sample geometry (Fig. 4).

For compression tests carried out inside TEM, one of the main concern is always whether the high energy electron beam (e-beam) affect the measured mechanical properties or not. The answer is, for materials with metallic bond, no obvious effect has been observed so far [105]. However, for materials with covalent and ionic bonds, significant e-beam effect has been confirmed. One typical example is Zheng et al. [106] study on amorphous $\mathrm{SiO} 2$ spheres. As they found during the compression test at room temperature, the flow stress can be decreased by four times when the e-beam is illuminating the sample comparing the condition e-beam is shielded from the sample (Fig. 5) [105].

With the advent of the atomic force microscope (AFM) and specialized depth sensing indenters, the probing of mechanical properties on the micro- and nano-scale under ultra- low loads has become possible [33]. This advancement in technology has proven useful for understanding the mechanical 


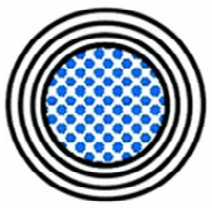

a

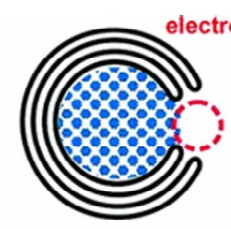

b

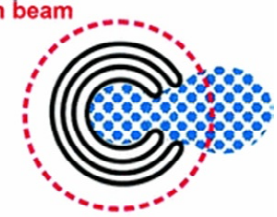

C
Fig. 6. Mechanism of the experiment used by Sun et al. [115] to study deformation of nano sized metal crystals: Graphitic shells encapsulating a metal crystal (a) are punctured by a focused electron beam (b). Successive electron irradiation of the container leads to the contraction of the shells and extrusion of the metal crystal (c). The red circles indicate the diameter of the electron beam. Reprinted with permission from Sun LT, Krasheninnikov AV, Ahlgren T, Nordlund K, Banhart F. 2008. Plastic Deformation of Single Nanometer-Sized Crystals. Physical Review Letters 101, [115]: (c) American Physical Society.

behavior of micro- and nano-scale materials ${ }^{107-112}$. As an example, a Hysitron SPM-3800 Atomic Force Microscope (AFM) with a pyramidal-shape Berkovich diamond indenter was used by Gan et al. [113], to perform the nanoindentation measurements of the individual phases. Wood et al. [114] used AFM nanoindentation to examine the local plasticity and size dependent hardness of bimetallic Ni-Au NWs with small dimensions attached to chemically functionalized flat substrates.

In a different method than all the mentioned experimental approaches for nano-compression, Sun et al. [115] designed graphitic cages that contract under electron irradiation and used them as nanoscopic deformation cells for in situ electron microscopy observations of the plastic deformation of individual nanometer-sized $\mathrm{Au}, \mathrm{Pt}, \mathrm{W}$, and $\mathrm{Mo}$ crystals. The principle of their experiment is shown schematically in Fig. 6.

\subsection{Sample fabrication and its effect}

Similar to the bulk compression test, sample is preferred to be cylindrical in shape, for load displacement to stress strain conversion. Moreover, a TEM sample must have a specific geometry, such as a free half-space (Fig. 4b) in front of the electron-transparent zone. The use of cylindrical nanopillars with a constant section deformed by flat punch tips is the easiest way to measure stress from load values $[28,35,116]$.

Experimental techniques such as electron-beam lithography [117-123], scanning tunneling microscopy [124,125], scratch lithography [126] with atomic force microscopy [127], cluster-beam deposition of metallic etch masks [128-130], nanosphere lithography [130-138], laser irradiation [139-141] have been used to fabricate nano or micropillars. However, not all of them are suitable for the fabrication of nano and micro-compression samples, mainly because they don't make pillars with the uniform cross section along the sample height. Moreover, some of the mentioned techniques are difficult to be applied for metallic pillar fabrication.

Following the approach of fabricating micropillars by utilizing focused ion beam (FIB) machining to etch patterns of interest, which was developed by Uchic et al. [35] and used by Greer et al. [36], Greer and Nix [6] extended this technique to much smaller pillars in the scale of nano. The fabrication of nanopillars by FIB is relatively easy, site-specific and can be applied to a wide range of materials. The principles of pillar fabrication with this method has been described in [142,143], and [144]. Till now, many scholars have used this method to fabricate sub-micron and nanopillars $[6,25,28,40,41,49$, 94,145-148].

Although FIB milling became a key technology in small scale mechanics, its side effects raise serious concerns about the validity of mechanical data. When the $\mathrm{Ga}^{+}$ions hit the sample surface during FIB imaging or milling process, structural defects such as point defects and dislocation loops, or even amorphization are induced into the surface due to the knock-on displacement and/or Ga+ implantation $[149,150]$, as revealed by transmission electron microscopy [28,151-154], X-ray diffraction [155] and atom probe tomography [156]. The FIB effect on dislocation density cannot be ignored when plasticity of small scales is studied and is already known to significantly affect the mechanical properties [25]. The thickness of the damaged layer could be around 10 to $100 \mathrm{~nm}$, depending on the material and experimental conditions $[150,152,156]$. Therefore, compression tests of sub-micron and nanopillars fabricated by FIB milling, may measure the composite mechanical response of a material and its FIB-modified surface rather than measuring the intrinsic properties of a material [146]. According to Lee et al. [157], surface damage induced by FIB irradiation, such as surface roughness or injected dislocation loops, would reduce the yield strength of a defectfree crystal, since facilitates dislocation nucleation at surface. The effect of $\mathrm{Ga}^{+}$implantation is not limited to mechanical properties, but can also change the physical properties and the thermodynamic stability [149]. Therefore, compression tests of 


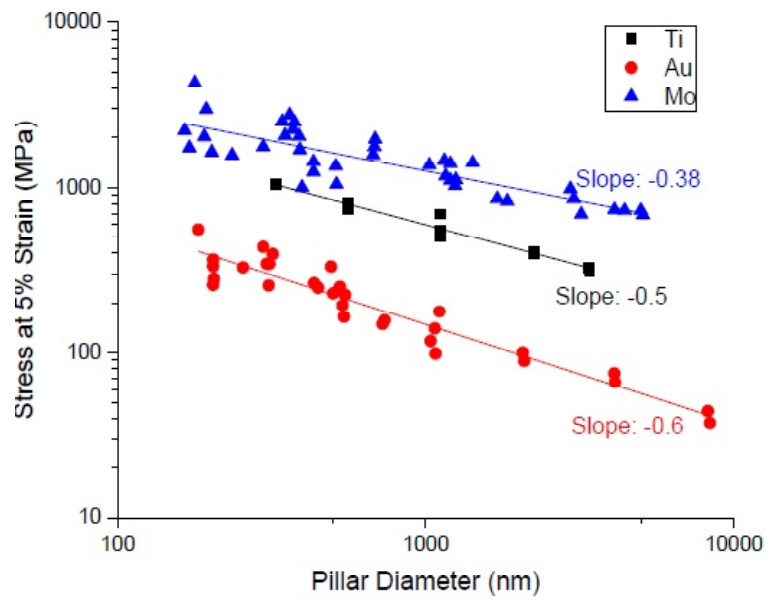

Fig. 7. Size effect on the strength of FIBed pillars (strength is taken as the flow stress at $5 \%$ plastic strain). Adapted from [55] and [60].

nanopillars fabricated by FIB milling, rather than measuring the intrinsic properties of a material, may measure the composite mechanical response of a material and its FIB-modiûed surface. Moreover, the inûuence of this damaged surface layer increases with decreasing specimen size. Hence, characterizing the effects of the FIB damaged layer on mechanical properties is important in interpreting the results of nanopillar tests. Remarkably, pillars prepared by FIB milling, exhibit a strong size dependent strengthening and as the pillar diameter becomes smaller, its flow stress increases as a power law: $\sigma=d^{n}$, where $d$ is the pillar diameter and $n$ lies between 0.6 and 1 for fcc metals [25,30,31,36,41,51,74,94,145,158-163], 0.22 and 0.45 for bcc $[30,41,48,55,58,94,160,164-169]$. Hex- agonal close-packed materials also appear to follow a power law, but with a lack of data the scaling behavior is less clear [66,165,170-172] and very dependent to the loading orientation so that Aitken et al. [170] found $n$ to be 1.11 for AZ31 nanopillars oriented for basal slip and 0.36 for those compressed along the $c$-axis. The mechanical size effect on $\mathrm{Au}$ (fcc), Mo (bcc) and Ti (hcp) is shown in Fig. 7, which illustrates the stress at $5 \%$ strain as a function of diameter. Because of possible slight misalignment in the initial stages of deformation, ûow stresses at $2.5 \%$ to $10 \%$ strain and when full contact is established, have been compared in papers rather than yield stresses, which cannot be unambiguously deûned in the experiments [37].

Shim et al. [173], showed that by doing ion bombardment parallel to the surface (glancing incidence), the range of penetration of $\mathrm{Ga}$ ions into the surface can decrease significantly, compare milling with normal incident angle (Fig. 8).

However, it was shown that other mechanisms such as "mechanical annealing" can remove this ion damage [28]. Greer and Nix [6] decreased the $\mathrm{Ga}^{+}$ion concentration by removing the conformal surface layer by etching the rotating pillar in lowenergy $\mathrm{Ar}^{+}$plasma and annealing. Lee at al. [174], showed that dislocation loops formed by the highenergy $\mathrm{Ga}^{+}$ion beam impact near the surface of sub-micron Al pillars can be removed by in-situ TEM annealing at around half of the melting point $\left(T_{\mathrm{m}}\right)$.

Burek and Greer $[175,176]$ used Electron Beam Lithography coupled with Electroplating technique to create isolated, vertically oriented, and virtually taper-free metallic nano-scale mechanical testing
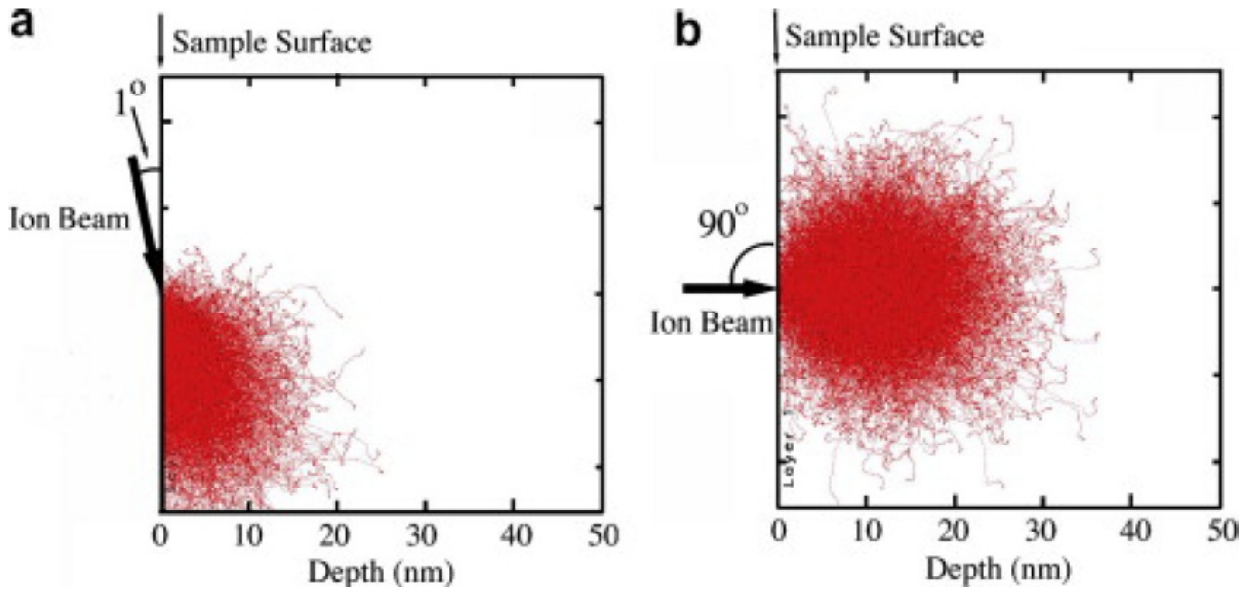

Fig. 8. Results of stopping and range of ions in matter (SRIM) calculations for $30 \mathrm{keV} \mathrm{Ga}$ ion penetration into pure Mo at (a) glancing incidence $\left(1^{\circ}\right)$ and (b) normal incidence $\left(90^{\circ}\right)$. Reprinted with pesrmission from Shim S, Bei H, Miller MK, Pharr GM, George EP. 2009. Effects of focused ion beam milling on the compressive behavior of directionally solidified micropillars and the nanoindentation response of an electropolished surface. Acta Materialia 57: (c) 2009 Elsevier Ltd. 


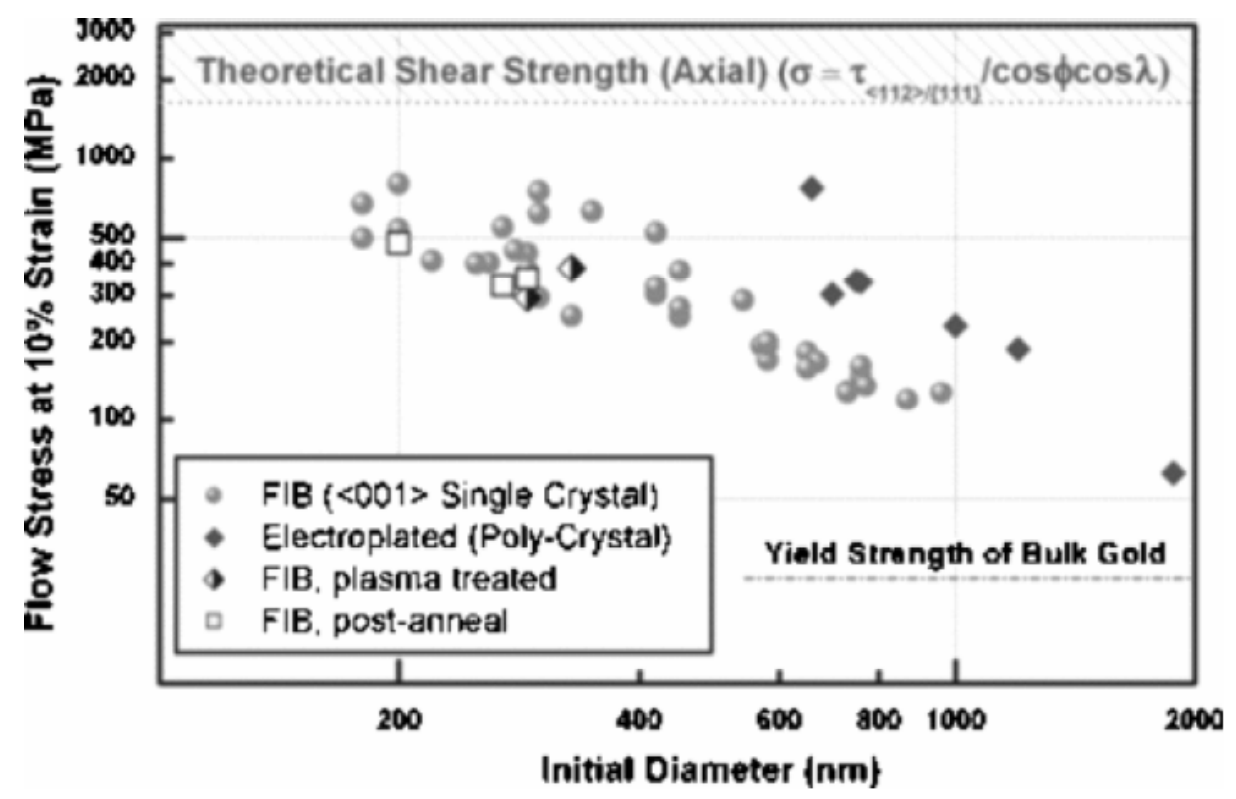

Fig. 9. Flow stress vs pillar diameter for the FIBed, electroplated, annealed, and Ar plasma-treated pillars compared with the range of theoretical strength and the yield strength of bulk Au. Reprinted with permission from Greer JR, Nix WD. 2006. Nanoscale gold pillars strengthened through dislocation starvation. Physical Review B 73, [6]: @2006 American Physical Society.

specimens with diameters from 750 down to $25 \mathrm{~nm}$. According to Burek and Greer [175], this fabrication method allows control over the pillar microstructure from nanocrystalline to single crystalline. The process had already been used by Greer et al. [6,36] to fabricate micron and nano-scale gold pillars and investigate the effect of fabrication process on size effect behavior at these scales. Fabrication method involves lithographic patterning of polymethylmethacrylate (PMMA) resist with electron beam, following by metal electrochemical deposition into the prescribed resist template. Fundamentally, this fabrication approach is analogous to other templatebased syntheses wherein one-dimensional nanostructures are fabricated by electroplating into the pores of either polycarbonate or alumina membranes. As Burek and Greer [175] claimed, the process has several advantages over the conventional FIB milling of nanopillars. At first, by replacing FIB fabrication with a procedure that does not utilize ion bombardment, the metallic nanopillar crystalline structure is absent of $\mathrm{Ga}^{+}$ion damage. At second electroplating makes it possible to fabricate pillars smaller than $100 \mathrm{~nm}$, which is difficult using other methods $[52,76,177]$. Third, when optimized this fabrication method greatly reduces pillar tapering relative to FIB fabrication and virtually eliminates the presence of any appreciable strain-gradients in the deformation process. Finally, the FIB fabrication process is inherently slow and significantly limits the sample throughput time. Moreover, the process can be easily applied for the fabrication of more complicated geometries like hollow cylindrical nanopillars [69].

According to Greer and Nix [6], the electroplated pillars, which were never subjected to the $\mathrm{Ga}^{+}$ions, not only have flow stresses higher than bulk metal but also exhibit a similar rise in strength as the diameter is reduced (Fig. 9). As they observed, the $\mathrm{Ga}^{+}$removal and pretest-annealing resulted in data that falls on the curve formed by the FIB pillars. Jennings and Greer $[52,76]$ fabricated Cu nanopillars without the use of $\mathrm{Ga}^{+}$and containing several initial dislocations, with electroplating technique and observed an identical size effect to the ones fabricated by FIB (Fig. 10). As Greer and Nix [6] concluded, the observed size effect in small scale is not linked to a specific fabrication technique. While some minimal $\mathrm{Ga}^{+}$might be present on the surface of the pillars, it is not a major contributing factor in the strength increase. The same conclusion was reached by Dietiker et al. [178], as they compared the behavior of pillars fabricated by FIB machining and nanoimprinting. However, as Kiener et al. [103] pointed out, length scale depends on the defect characteristics in the material. They irradiated oriented copper single crystal using a $1.1 \mathrm{MeV}$ proton beam at near-ambient temperature and subsequently FIB machined nano-compression samples and performed quantitative in situ tests in a TEM to 


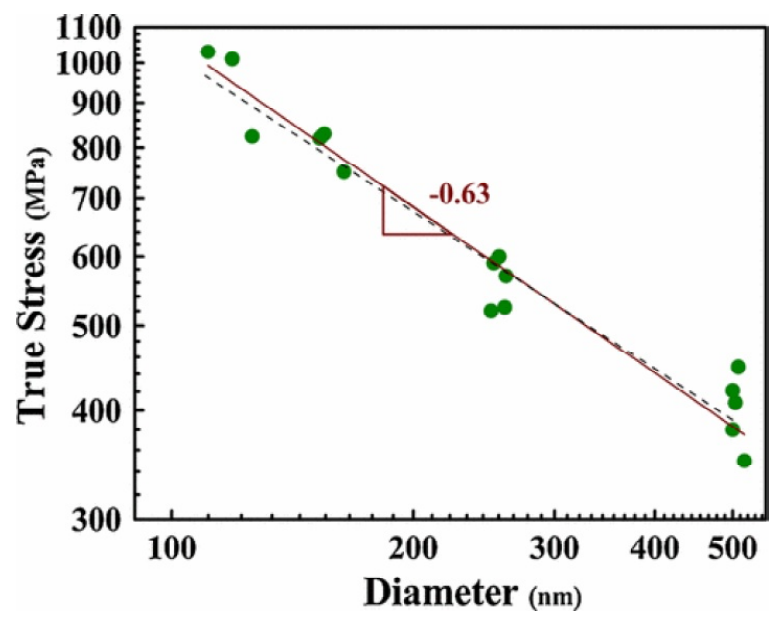

Fig. 10. Flow stress at $10 \%$ strain for Cu nanopillars fabricated by electroplating, presenting a power-law trend with $n=" 0.63$, nearly identical to the trend demonstrated in FCC metals represented by the solid line. Reprinted with permission from Jennings AT, Burek MJ, Greer JR. 2010. Microstructure versus Size: Mechanical Properties of Electroplated Single Crystalline $\mathrm{Cu}$ Nanopillars. Physical Review Letters 104, [52]: @2010 American Physical Society.

investigate the influence of irradiation defects on the material properties (Fig. 11). As they observed, while the non-irradiated samples exhibited a size dependent behaviour over the entire size ranges, the irradiated ones followed this trend only for specimen diameters up to $\sim 400 \mathrm{~nm}$. They concluded that this transition length scale depends on the defect characteristics and therefore on the material, irradiation dose and temperature. For the irradiated samples, deformation was observed to be strongly localized due to the requirement of forming a large enough dislocation source in a volume containing densely spaced irradiation defects.

\section{SMALL SCALE DEFORMATION MECHANISMS}

\subsection{FCC metals}

For typical face-centered cubic (FCC) metals like $\mathrm{Ag}, \mathrm{Au}, \mathrm{Cu}, \mathrm{Ni}$, and $\mathrm{Al}$ with various stacking fault energy values ( 22, 45, 78, 128 and $\sim 166 \mathrm{~mJ} /$ $\mathrm{m}^{2}$, respectively) [179], it was found that when the sample size is reduced to nano-scale, the mechanical properties such as yield strength and plasticity would both increase $[6,35,179,180]$.

Fig. 12 shows examples of the mechanical properties and flow behavior of sub-micron scale single crystalline gold and nickel pillars. The most obvi- ous difference between pillars, whiskers, and bulk single crystals is the observed stress for plastic flow. Bulk single crystals deform at very low strengths, on the order of 10s of MPa. Micro and nanopillars range in strength from bulk-levels at large pillar diameters to gigapascals at $200 \mathrm{~nm}$, as seen in Fig. 12; however, the strengths for the smallest pillars are still significantly lower than similar size whiskers which have near theoretical strengths [181]. As is seen in Fig. 12, in the larger pillars, the stress strain curve is continuous and similar to that observed in a standard bulk compression tests; while, in smaller pillars with diameters below $\sim 1 \mu \mathrm{m}$, the deformation behavior is distinct from bulk tests and is characterized by discrete bursts of plasticity (popins [182]) corresponding to dislocation avalanches. Furthermore, after a dislocation avalanche, the pillar loads again until a subsequent dislocation avalanche occurs at a similar stress level.

\subsubsection{Dislocation starvation and nucleation control}

One of the most likely deformation modes that can result into the observed high strengths is explained by the concept of dislocation starvation. According to this scenario [6], in small volumes mobile dislocations are thought to escape from the crystal at the nearest free surface before multiplying and interacting with other dislocations. Such processes

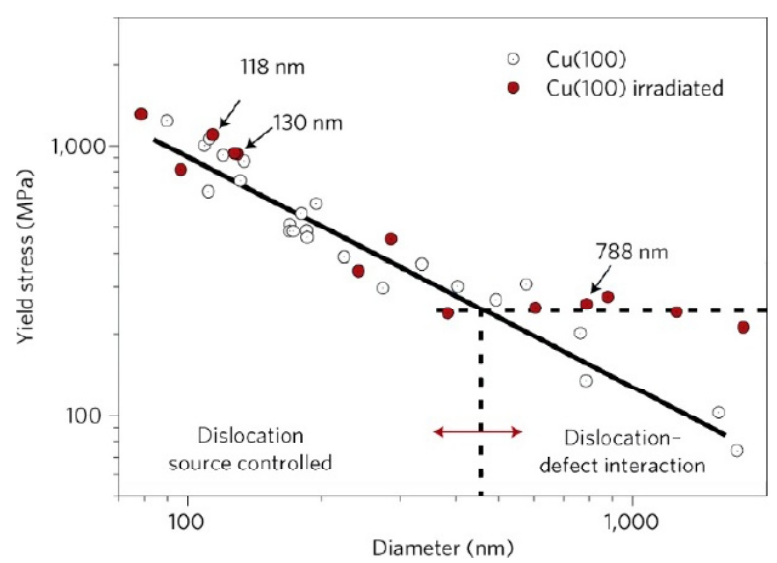

Fig. 11. Comparison of size dependent behavior of irradiated and unirradiated copper samples. While the unirradiated copper (open circles) exhibits a size dependent yield strength over the whole size range, the irradiated samples (red symbols) show this behaviour only when the size is less than $400 \mathrm{~nm}$. Reprinted with permission from Kiener D, Hosemann P, Maloy SA, Minor AM. 2011. In situ nanocompression testing of irradiated copper. Nat Mater 10, [103]: @2011 Nature Publishing Group. 

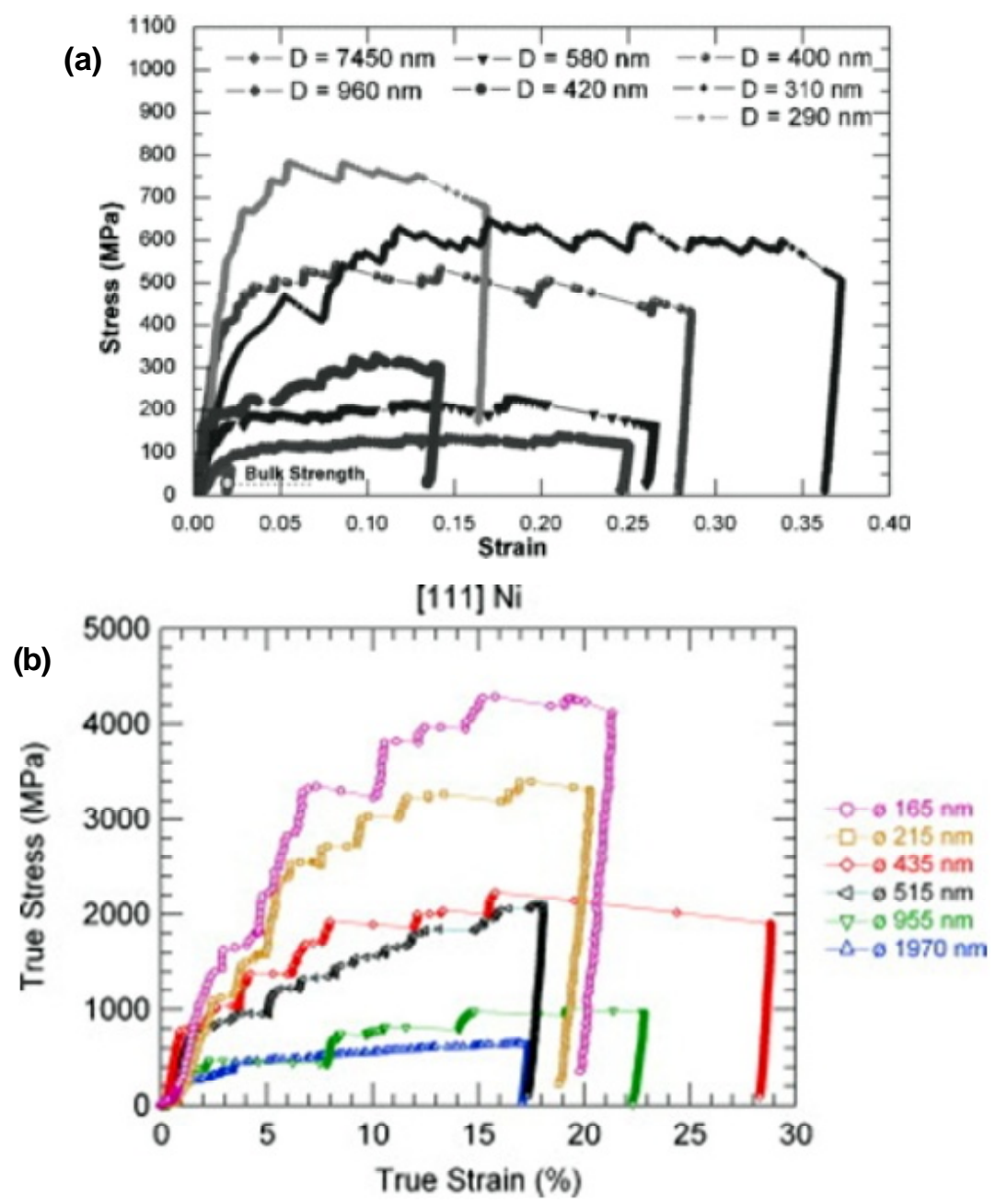

Fig. 12. Stress-strain behavior of (a) $<001>$ oriented FIBed Au and (b) $<111>$ Ni pillars of different diameters, $[6,145]$. Reprinted with permission from Greer JR, Nix WD. 2006. Nanoscale gold pillars strengthened through dislocation starvation. Physical Review B 73 (6), @2006 American Physical Society. Reprinted with permission from Frick CP, Clark BG, Orso S, Schneider AS, Arzt E. 2008. Size effect on strength and strain hardening of small-scale [111] nickel compression pillars. Materials Science and Engineering A 489 (147): (c) 2008 Elsevier Ltd.

lead to a dislocation-starved state, and nucleation of new dislocations in the newly-formed perfect crystal is required to accommodate further plastic deformation. According to Greer et al. [32,36,183,184] description, a dislocation must travel a minimum distance, $\delta$ (also called breeding distance) before it replicates itself, and when crystals is smaller than this characteristic length, it might behave quite differently from bulk crystals. When the conditions for multiplication are not met, the dislocations would leave the small crystals before having the chance of multiplication, leading to dislocation starvation. Once the dislocation-starved conditions are reached, very high stresses would be required to nucleate new dislocations, either at surfaces or in the bulk of the crystal, which could describe the observed neartheoretical-strengths at small scales.
Fig. 13 shows Shan et al. [28] results as they evaluated the dislocation starvation theory. As they observed, the dislocation content of the nanopillars dropped markedly when deformed in the TEM. With this exhaustion, which the authors call 'mechanical annealing', the process controlling strength and plasticity changes from dislocation propagation and interaction to dislocation nucleation [116].

Jerusalem et al. [185], proposed a continuum model accounting for dislocation starvation and nucleation. As they described, a reference of the average dislocation resistance shear stress for each slip system i can be describes as,

$$
\tau_{0}^{i}=\min \left(\left(1-\frac{\varepsilon_{\rho}}{\varepsilon_{\rho}^{\text {starv }}}\right) \tau_{0, \text { starv }}+\frac{\varepsilon_{\rho}}{\varepsilon_{\rho}^{\text {starv }}} \tau_{0, \text { nucl }}, \tau_{0, \text { nucl }}\right),
$$



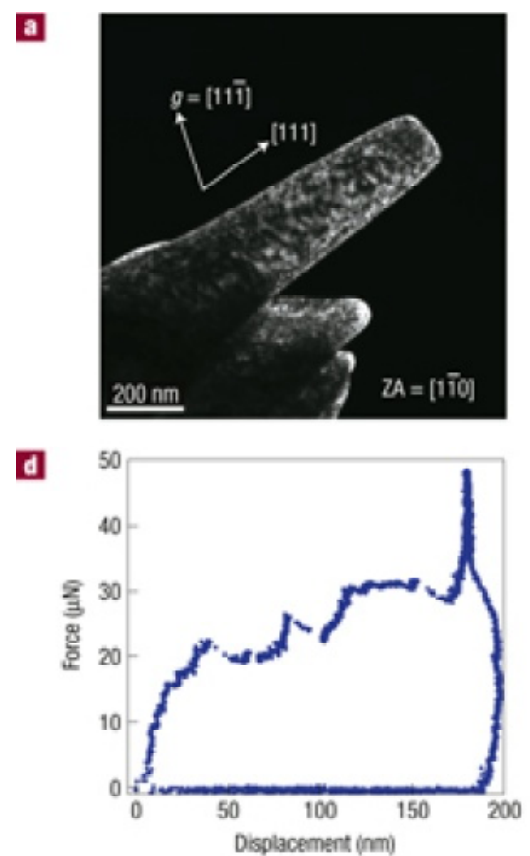

b

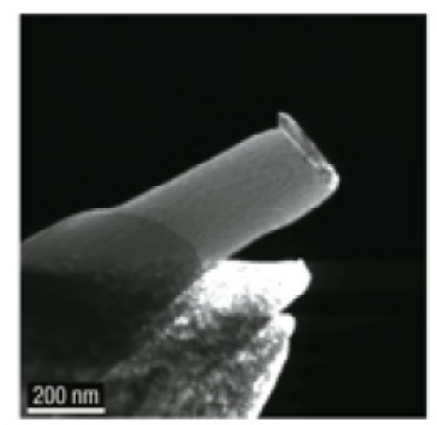

曰

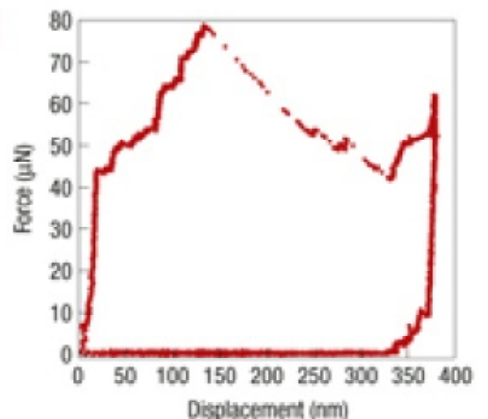

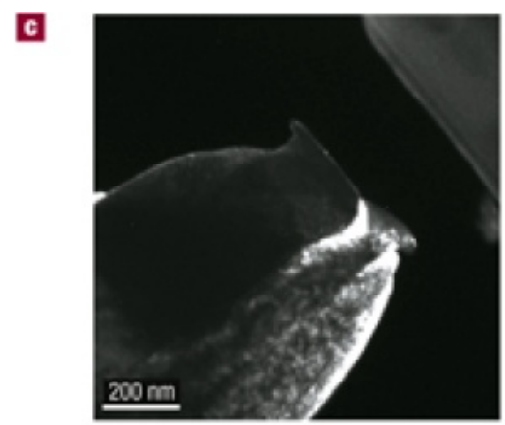

I.

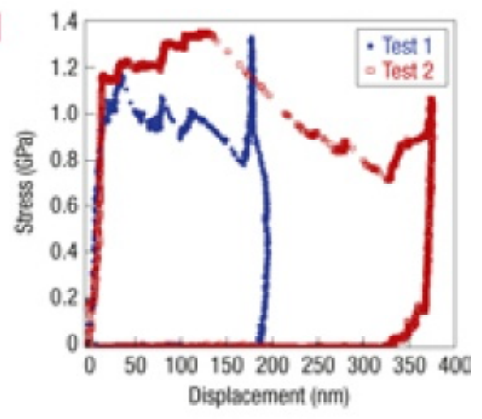

Fig. 13. In situ TEM compression of $<111>$ oriented $160 \mathrm{~nm}$ Ni pillar. a) Dark-field TEM image of the pillar before the test, showing high initial dislocation density, b) Dark-field TEM image of the same pillar after the first test which is now free of dislocations, c) Dark-field TEM image of the same pillar after the second test. d) Force versus displacement curve of the first and e) second test. f) Instantaneous stress versus compressive displacement for the two tests; the apparent yield stress is similar for both tests. Reprinted with permission from Shan ZW, Mishra RK, Asif SAS, Warren OL, Minor AM. 2008. Mechanical annealing and sourcelimited deformation in submicrometre-diameter Ni crystals. Nature Materials 7, [28]: @2008 Nature Publishing Group.

where

$$
\tau_{0, \text { stav }}=0.5 \mu b \sqrt{\rho_{0}}+1.4 \frac{\mu b}{4 \pi a_{0}(1-v)} \ln \left(\frac{\alpha a_{0}}{b}\right) .
$$

and $\varepsilon_{\rho}^{\text {star }}$ considers the plastic strain for which nucleation dislocation is more favorable than dislocation starvation, and at which the nucleation critical resolved shear stresses (CRSS) $\tau_{0, \text { nucl }}$ is reached.

According to Zhu et al. [186] theory, the required stress for dislocation nucleation is proportional to the logarithm of the number equivalent surface nucleation sites; $N$, which is directly affected by the sample geometry. Since nucleation stress is a function of logarithm of $N$, the size effect arising from surface nucleation is expected to be weak, compared to that in micropillars showing a power-law scaling exponent in the range of " 0.6 to " 0.7 [31,36,50,51,145]. As Zhu et al. [186] assumed, a transition in the scaling behavior of yield stress could be expected on the sample size in an approximate range of tens of nanometers, as illustrated by Fig. 14.

TEM studies on partial dislocation nucleation have highlighted the role of various surface defects in dislocation nucleation. This is most obvious in the case of thin films where dislocations preferentially nucleate at voids as they serve as stress concentrations sites during tensile experiments $[187,188]$. The effect of surface steps on decreasing the elastic threshold and assisting the nucleation of dislocations [189-197]. As Navarro et al. [197] calculated, the critical stress for dislocation nucleation at the steps is approximately half that on the flat surface.

Large strain bursts were universally observed during the compression of sub-micron and nanosized fcc pillars. However, depending sample size, the underlying physical mechanisms are different. As Wang et al. [198] concluded on his studies on Al pillars, for small pillars (with $D=80$ to $300 \mathrm{~nm}$, group $A$ ), the bursts originate from explosive and highly correlated dislocation nucleation, characterized by very high ultimate stresses and nearly dislocation-free microstructure after the sample failure. Fig. 15 shows a $165 \mathrm{~nm}$ Al pillar as an example of this group. The single crystal is loaded along the

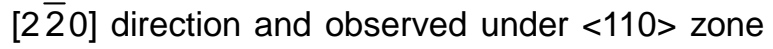
axis. FIB induced dislocations are clearly seen in the darkfield image (Fig. 15c). The first burst ob- 


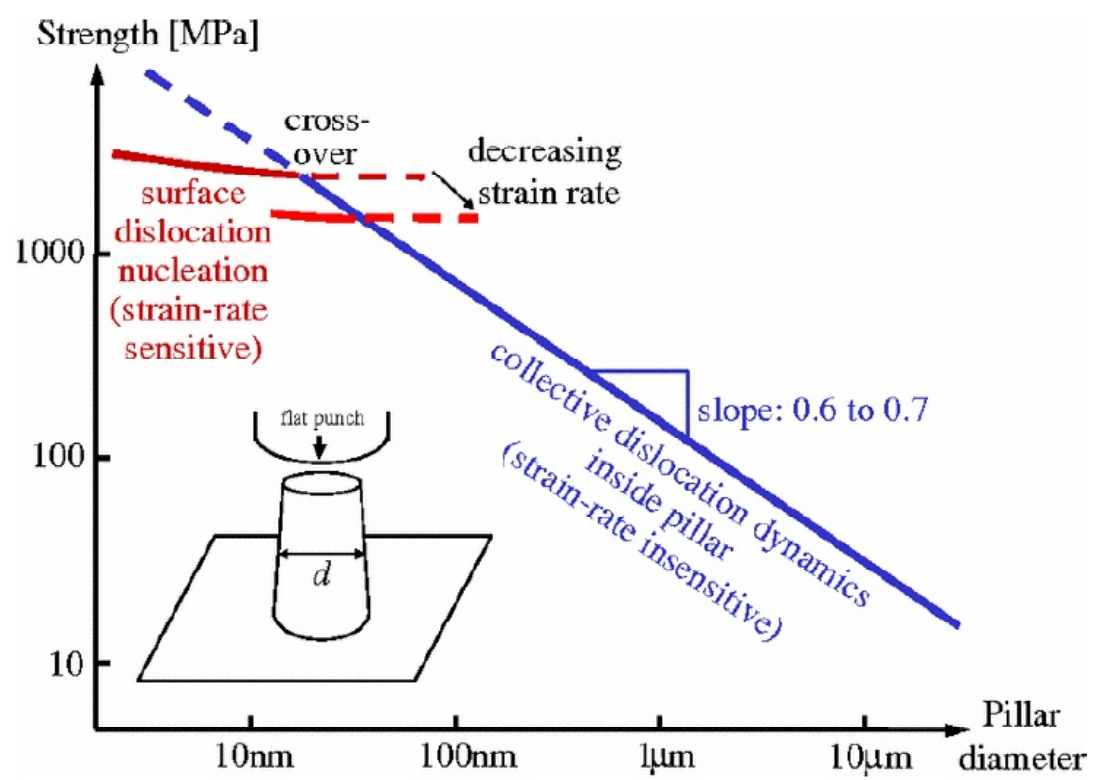

Fig. 14. Transition in the scaling behavior of yield stress on the sample size range of tens of nanometers. Reprinted with permission from Zhu T, Li J, Samanta A, Leach A, Gall K. 2008. Temperature and strain-rate dependence of surface dislocation nucleation. Physical Review Letters 100, [186]: (c) American Physical Society.
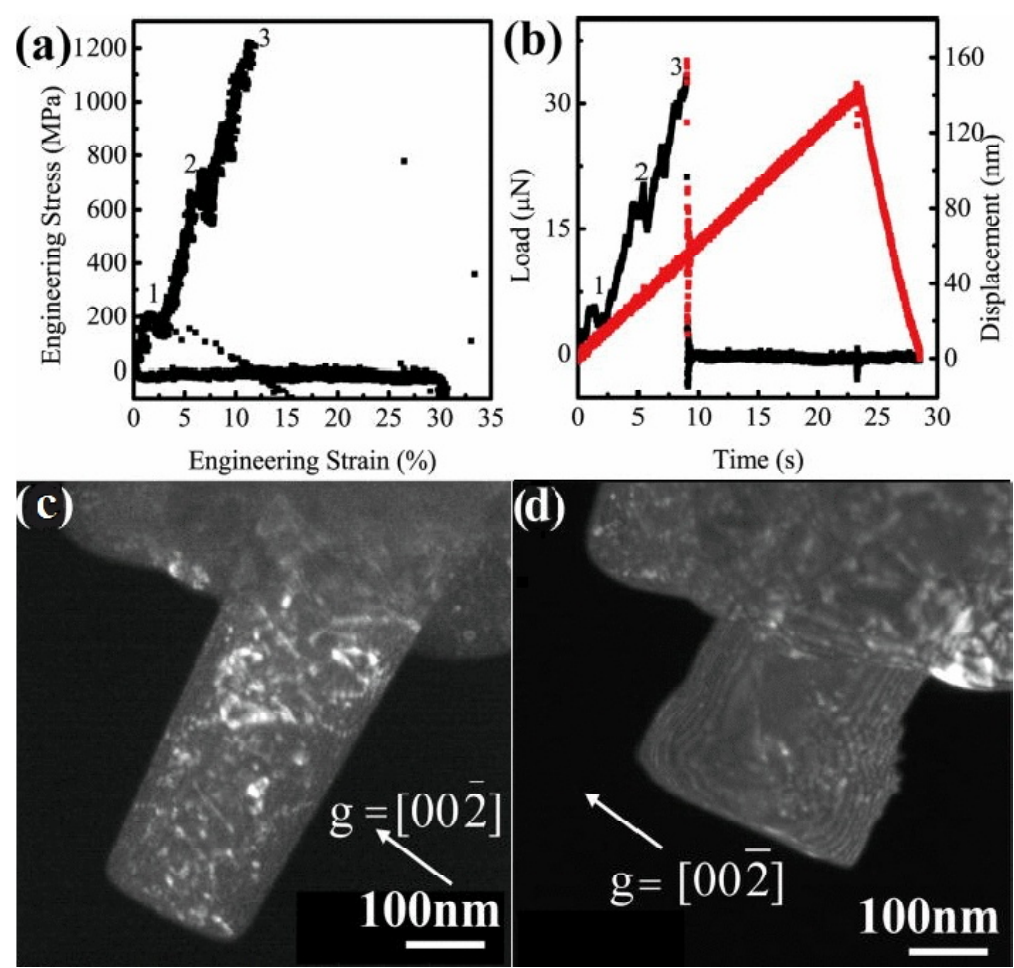

Fig. 15. Snapshots of the microstructural evolution in $165 \mathrm{~nm}$ Al nanopillar compression. (a) Engineering stress-strain curve (b) The load-time and displacement-time curves. (c) Dark-field images of the pillar before compression. (d) Dark-field images of the pillar after collapse ( 20\% strain). Reprinted with permission from Wang Z-J, Li Q-J, Shan Z-W, Li J, Sun J, Ma E. 2012. Sample size effects on the large strain bursts in submicron aluminum pillars. Applied Physics Letters 100, [198]: @ 2012 AIP Publishing LLC.

served in the stress strain and load time curves (Figs $15 a$ and $15 b$ ) is caused by the cleaning-up of preexisting dislocation which were driven out of the pillar, in a process known as mechanical annealing
$[25,28,101,116,198,199]$. Here, the CRSS is equal to the starvation CRSS in equation 1 and then linearly increases as a function of $\varepsilon_{\rho}$ as the mobile dislocations are moving towards the free surface. 


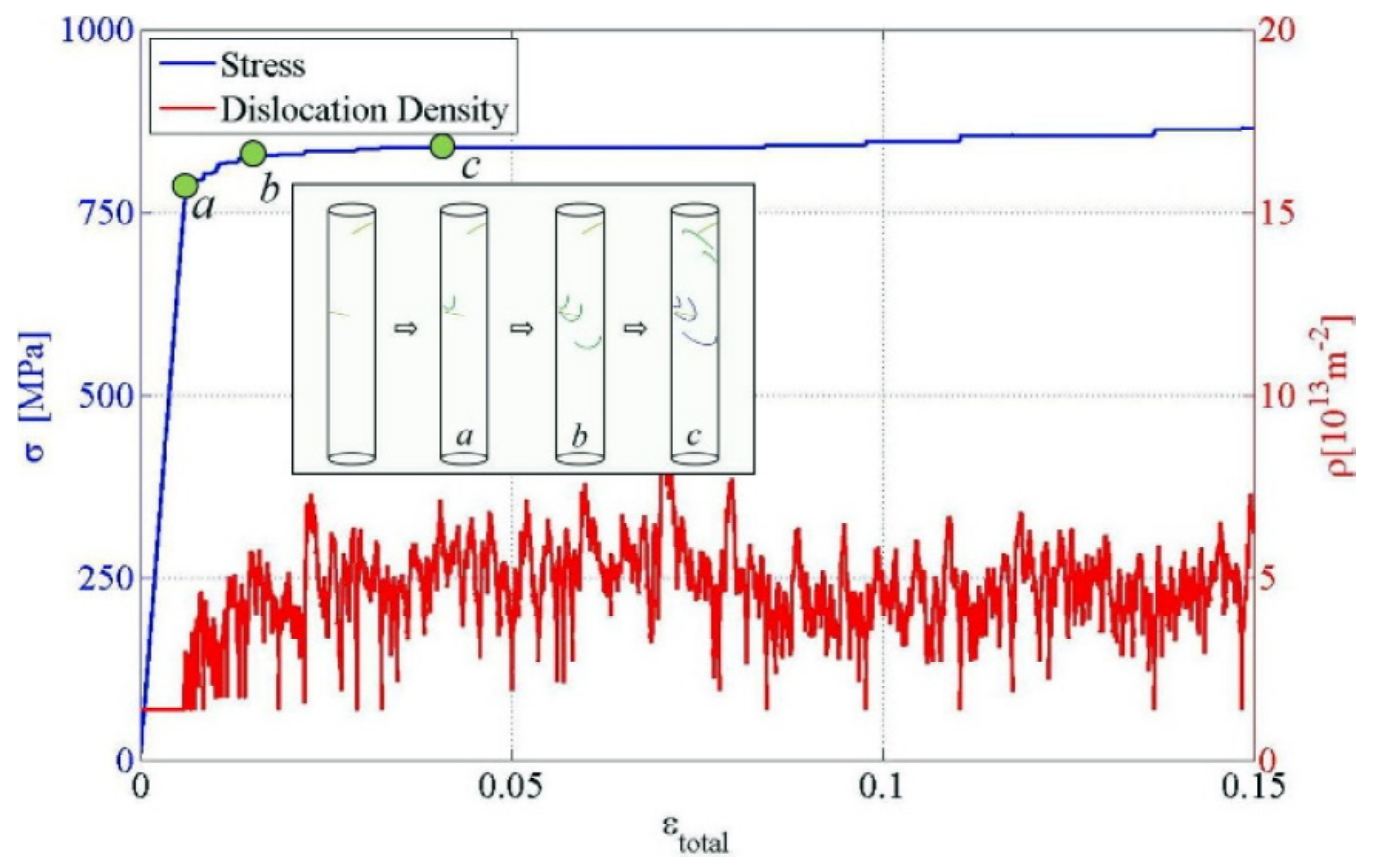

Fig. 16. Stress and dislocation density evolution versus strain for a $150 \mathrm{~nm}$ diameter Cu pillar. Insets show the dislocation microstructures at different points along the stress-strain curve. Reprinted with permission from Ryu I, Cai W, Nix WD, Gao H. 2015. Stochastic behaviors in plastic deformation of face-centered cubic micropillars governed by surface nucleation and truncated source operation. Acta Materialia 95, [202]: (c) 2015 Elsevier Ltd.

Once all mobile dislocations are annihilated, plasticity is fully nucleation driven and second drop at stress, marked point 2, happens when new dislocations nucleate from the contact interface between the pillar and the indenter. However, since there is no dislocation in the pillar, the new ones move through it very fast and escape immediately out of the pillar. The drastic strain burst happens in point 3 , where the structural collapse occurred, in result of a major shape change within a small fraction of a second [198]. Post-test, dark-field observation under multiple conditions confirmed that this test left behind a dislocation-free pillar (Fig. 15d) $[28,198]$. The pronounced decrease in dislocation density during the nano-compression tests provides direct support for the dislocation starvation theory that has been hypothesized based on experiments $[6,30,32,36,94]$ and simulations $[38,185,186,200]$. As Shan et al. [28] pointed out, even the surface oxide layer [201] and the FIB damage layer [152], cannot trap dislocations inside the pillar during deformation [105]. The driving force for the dislocation starvation within the pillars is a combination of the applied stress which will activate or nucleate dislocations and the image forces from the surface which will assist the dislocations in moving towards the free surfaces of the crystal [28]. The mechanical annealing phenomena is more signiûcant in smaller the samples [105].

Fig. 16 shows the result of a dislocation dynamics (DD) simulation on the behavior of a $150 \mathrm{~nm} \mathrm{Cu}$ pillar in which all dislocations escape the crystal during the early stages of loading and half loop dislocations are introduced into the crystal from the surface, when the stress reaches the critical dislocation nucleation stress. If the applied load is not drop, then these new dislocations will traverse the pillar and subsequently escape and this process is then repeated with the flow stress reaching a plateau without any significant hardening [202].

\subsubsection{Single arm dislocation source control}

Some scholars have proposed a mechanism based on the operation and stochastic effects of a single arm dislocation source within a pillar in the scale of micrometer, believing that the micropillars contain several dislocation sources, but only one or two for the slip plane in the small pillars $[153,203]$. As Parthasarathy et al. [203] described, in finite samples, where the sample dimensions are of the same order of magnitude as the source lengths, all sources end up as being single-ended due to interaction with 


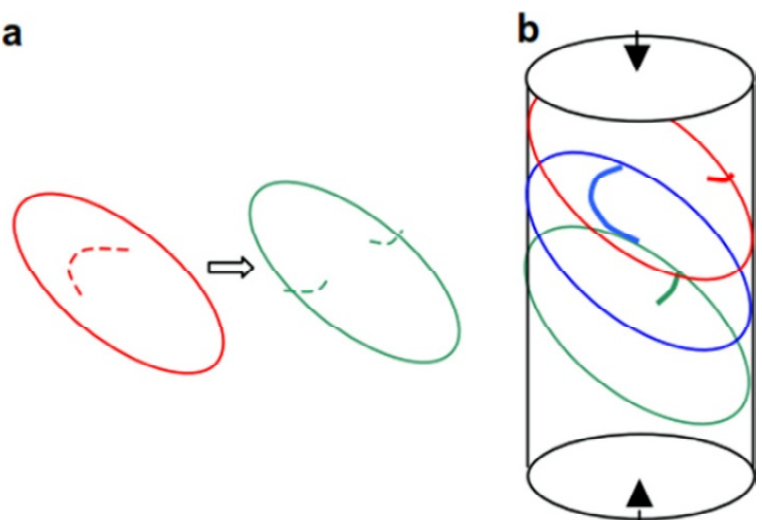

Fig. 17. (a) A schematic description of changing a double-pinned Frank-Read sources to single arm sources in a small volume. (b) Representation of single arm sources in a finite cylindrical sample in critical configuration, which occur where the distance from the pin to the free surface is the shortest. The longest arm determines the critical resolved shear stress. Reprinted with permission from Parthasarathy TA, Rao SI, Dimiduk DM, Uchic MD, Trinkle DR. 2007. Contribution to size effect of yield strength from the stochastics of dislocation source lengths in finite samples. Scripta Materialia 56, [203]: (c) 2015 Elsevier Ltd.

the free surface, as in Fig. 17b. Fig. 18 shows one of single arm sources operating in a $750 \mathrm{~nm}$ wide Al fiber [204]. In Fig. 18a, the spiral source is captured while generating dislocation loops in a (111) glide plane by turning clockwise around a fixed arm. The moving arm propagates to the right, and forms a pile-up because of the non-immediate evacuation of dislocations to the free surfaces. Thick slip traces (noted Tr. in Fig. 18a) are evidence of the slow shear of the native oxide by the dislocations. The slip traces are erased when the source stops by crossslipping of the fixed arm, meaning that the dislocations have completely escaped the crystal (Fig. 18b). This single arm mechanism has been found acceptable and used in discrete dislocation dynamic simulations [200,205-212]. Moreover, some physical models have been developed based on this stochastic effect, such as the single arm source (SAS) model [180,203,206,213,214]. Pan et al. [215], examined the dislocation pile-up mechanism quantitatively and provided a revised SAS model for describing the size dependent strength by reconsidering the back stress and activation stress.

The critical resolved shear stress to activate a single arm dislocation source is generally computed by summing the friction stress, the elastic interaction stress, and the line tension stress. Choosing
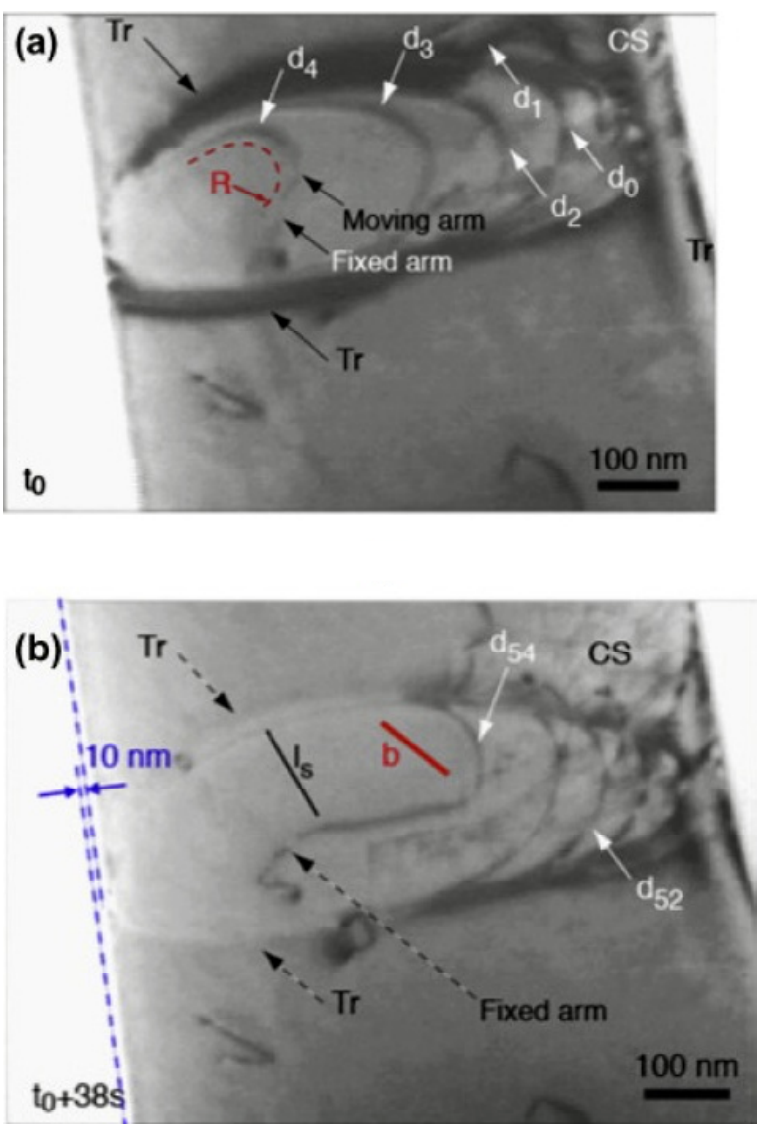

Fig. 18. Single arm source operating in a $750 \mathrm{~nm}$ wide Al fiber for a finite time (54 loop emissions in $38 \mathrm{~s}$ ). (a) and (b) are video frames that correspond to the source operating and stopping, respectively. Reprinted with permission from Mompiou F, Legros M, Sedlmayr A, Gianola DS, Caillard D, Kraft O. 2012. Source-based strengthening of sub-micrometer Al fibers. Acta Materialia 60, [204]: (c) 2012 Elsevier Ltd.

Parthasarathy et al. [203] equation and considering material parameters, Lee and Nix [180] expressed the required CRSS as,

$$
\tau_{\text {CRss }}=\tau_{0}+0.5 k_{s} b \sqrt{\rho_{\text {tot }}}+\frac{\alpha k_{s} b}{\bar{\lambda}_{\max }\left(D, \rho_{\text {tot }}, \beta\right)},
$$

where $\tau_{\text {CRSS }}$ is the CRSS for the activation of the weakest single arm source, $\tau_{0}$ is the friction stress, $k_{s}$ is the anisotropic shear modulus, $b$ is the magnitude of Burgers vector, $\rho_{\text {tot }}$ is the total dislocation density, $\alpha$ is a constant of the order of unity, and $\bar{\lambda}_{\text {max }}$ is the statistical average length of the weakest single arm dislocation source, $D$ is the pillar diameter, and $\beta$ is the slip plane orientation. As Lee and Nix [180] described, if all of the samples have a similar dislocation density and slip plane orientation, $\bar{\lambda}_{\text {max }}$ becomes only diameter dependent, which 
indicates the capability of SAS theory for describing size scale properties.

The stability of dislocation sources has been studied in the literature. Molecular dynamics simulations have shown that jogs on Lomer-Cottrell dislocations that might form the pinned part of single arm sources are unstable in the high stress loading conditions required to deform Al nanopillars of diameter 16-50 nm [216], while 3D discrete dislocation plasticity (DDP) simulations approved the importance of image stresses and cross-slip in controlling the lifetime of intermittent sources which tend to be longer lived in larger diameter samples [200]. However, TEM observations of deformation of Al fibers of diameter $120 \mathrm{~nm}$ confirm that although operating intermittently, an individual single arm dislocation source is sufficiently stable to generate large plastic strain [217].

Investigating single arm source controlled plastic ûow in the micropillars with diameters from 200 to $800 \mathrm{~nm}$ is extensively investigated by a statistically, Cui et al. [206] noted that the SAS mechanism itself is not suitable to explain the hardening behavior of very small or very large samples. As the sample size decreases, the image force becomes larger and together with the external applied stress, the dislocations can be easily driven out of the crystal, resulting that available dislocation sources become progressively exhausted, even causing dislocation starvation. The similar conclusion was made by Gu et al. [218], pointing out that in fcc single crystalline metals, dislocations are generated by the operation of the single arm dislocation sources in micrometer-sized structures and via dislocation nucleation at surfaces in nanometer-sized structures [214,218-220].
Hu et al. [221], studied the behavior 300 to 700 $\mathrm{nm}$ diameter gold particles and resulted that abundant dislocations nucleation and storage exist inside their 400 and $500 \mathrm{~nm}$ particles during the compression, while few dislocations nucleate inside the smaller particles, concluding that the accumulated emission mechanism controls the collapse of relative larger particles and correlated emission is dominant for the smaller ones. As Jennings et al. [52] stated, the same deformation mechanisms govern flow behavior of nano-scale samples regardless of fabrication methods.

Modifying Jerusalem et al. [185] model (Eq. (1) and considering SAS operation mechanism, Cui et al. [206], developed a more general theoretical model to quantitatively describe the sub-micron plastic behavior as follows,

$$
\begin{aligned}
& \tau=\min \left(\left(1-\frac{\varepsilon_{\rho}}{\varepsilon_{\rho}^{\text {star }}}\right)\left(\tau_{0}+\alpha \mu b \sqrt{\rho}+\frac{k \mu b}{\bar{\lambda}}\right)\right. \\
& \left.+\frac{\varepsilon_{\rho}}{\varepsilon_{\rho}^{\text {star }}} \tau_{\text {nucl }}, \tau_{\text {nucl }}\right),
\end{aligned}
$$

where $\varepsilon_{\rho}$ is the engineering plastic strain, and $\varepsilon_{\rho}^{\text {star }}$ is the plastic strain for which dislocation nucleation is more favorable than SAS operation, and at which the CRSS for dislocation nucleation $\tau_{\text {nucl }}$ is reached. Cui [222] summarized possible mechanisms of FCC single crystal plastic deformation in a phase diagram shown in Fig 19. As is described, dislocation starvation is dominant in smaller sample with lower dislocation density $\left(\sim 10^{12} \mathrm{~m}^{-2}\right)$. Continuing deformation, dislocations gradually escape from the crystal, and surface nucleation controls plasticity. By increasing the sample size, it becomes more difûcult

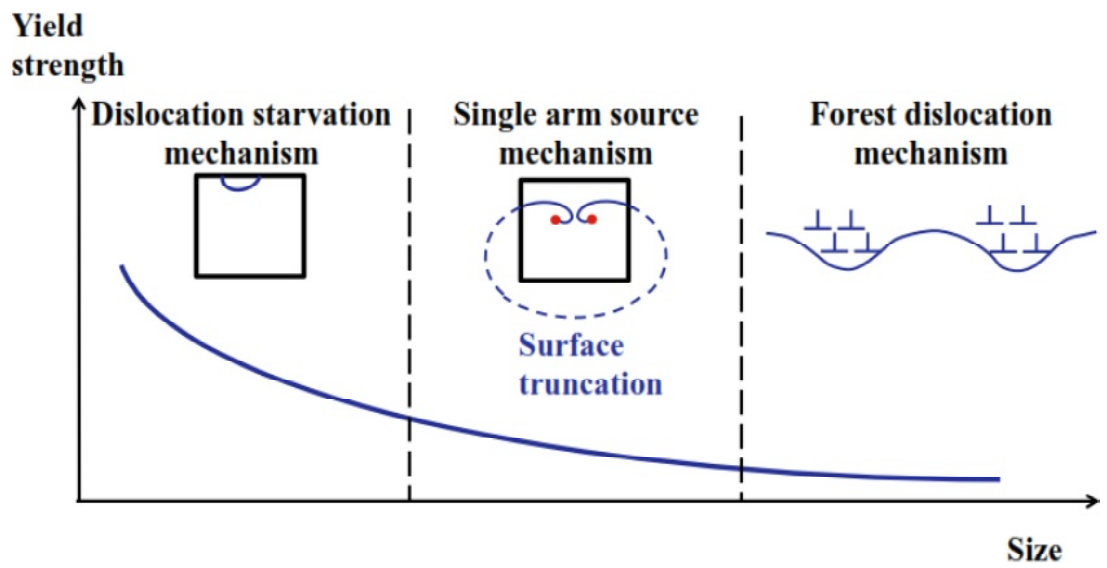

Fig. 19. Plastic deformation mechanisms in FCC single crystal. Reprinted with permission from Y. Cui 2017. The Investigation of Plastic Behavior by Discrete Dislocation Dynamics for Single Crystal Pillar at Submicron Scale, [222]: (c) Springer Singapore. 


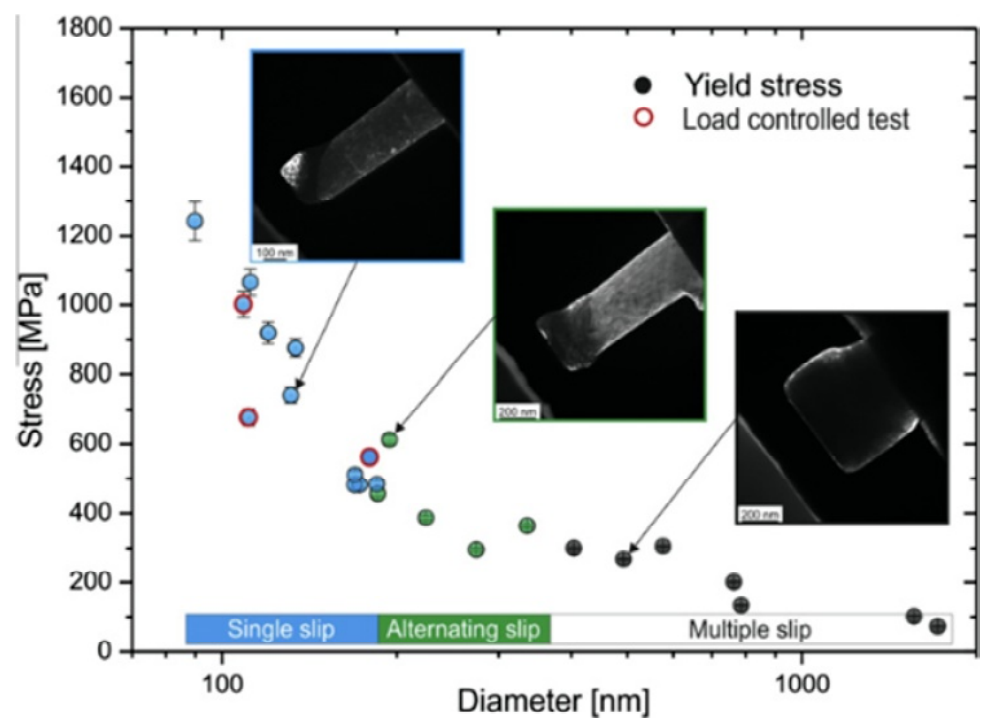

Fig. 20. Yield stress results of compressing $\mathrm{Cu}(100)$ samples ranging in diameter from $90 \mathrm{~nm}$ to $1700 \mathrm{~nm}$. Besides the size eûect, a change in the deformation morphology is shown indicated by differently colored symbols. Reprinted with permission from Kiener D, Minor AM. 2011. Source-controlled yield and hardening of $\mathrm{Cu}(100)$ studied by in situ transmission electron microscopy. Acta Materialia 59, [223]: (c) 2011 Elsevier Ltd.

to reach the dislocation starvation state and dislocation sources emerge because of the interaction between internal dislocations. Here, the activation of single arm source plays the main role. If the sample size further increases, classical dislocation multiplication mechanism controls the deformation. The critical size between different mechanisms depends on dislocation density and internal microstructure, as shown schematically in Fig. 19. While there is still no quantitative expression, it is generally believed that the critical size decreases with the decline of dislocation density [222].

As Kiener and Minor [223] pointed out, the activation of slip planes and changing deformation morphology form single slip to alternating slip and multiple slips regimes is dependent to the sample size as shown in Fig. 20.

\subsubsection{Liquid like deformation}

As size scales reaches to the order of $10 \mathrm{~nm}$ and smaller, a new regime of behavior emerges and curvature and surface stress effects become more important. As an example, the Laplace pressure in an Au cylinder with diameter of $10 \mathrm{~nm}$ diameter and isotropic surface is around $0.28 \mathrm{GPa}$ and this large pressure would have significant effects on both elastic and plastic properties [224]. Moreover, new irreversible deformation modes involving structural transitions would occur at these small size scales. A number of researches have been done on theses scales and the effective phenomena [44,104,115,186,225-230].

According to Sun et al. [231] findings, the shape change of sub-10-nm silver (Ag) nanoparticles at room temperature is dominated by surface diffusion, causing them deform like liquid drops but with a highly crystalline core, which is fundamentally different from displacive shear plasticity by dislocations. They used a set-up where the Ag nanocrystal was partially bonded to a tungsten tip that approached a counter $\mathrm{ZrO}_{2}$ surface. Fig. 21 shows a typical cycle of compression and stretching of a Ag nanocrystal with a base diameter of 9.8. As the authors observed, when the Ag nanocrystal was compressed against the oxide, it continuously flattened (Figs. 21b-21d), and continuously elongated when the tip was retracted (Figs. 21e and 21f). Moreover, they observed that when the nanocrystal detached from the tip it rapidly recovered the original facetted shape. Visualization of the lattice planes in the TEM images demonstrated that the nanocrystal remained as a perfect single crystal during the whole process. Importantly, authors demonstrated that the nanocrystal deformed by surface diffusion, as indicated by the atomic planes growth on its external surface and is fundamentally different from displacive shear plasticity by dislocations. These results confirmed by means of molecular dynamics simulations [232].

Although dislocation based plastic deformation is expected to become inactive below a critical par- 
a

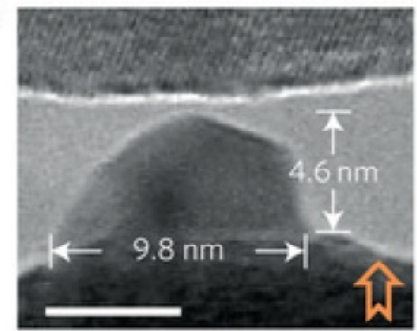

d

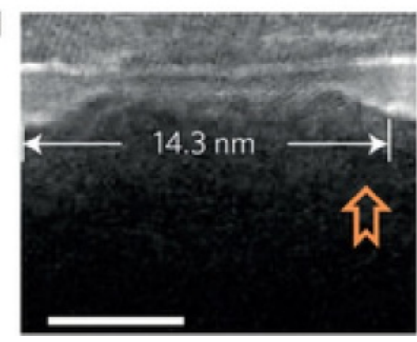

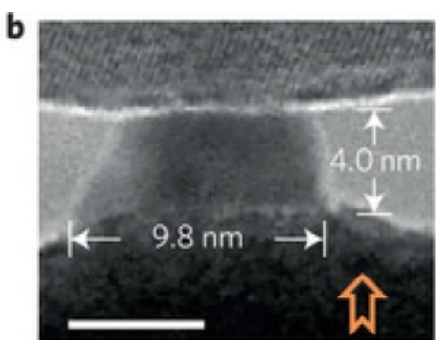

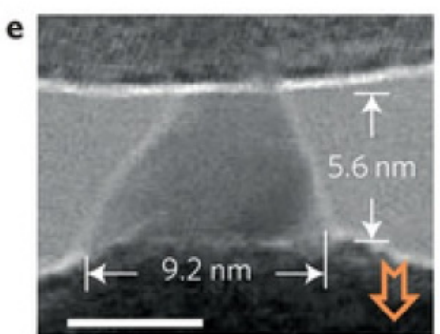

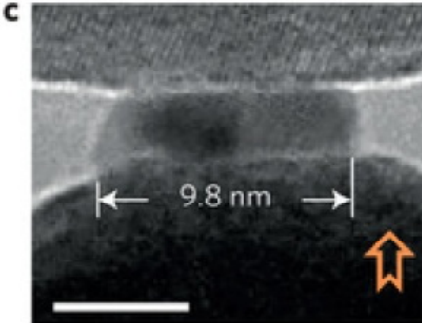

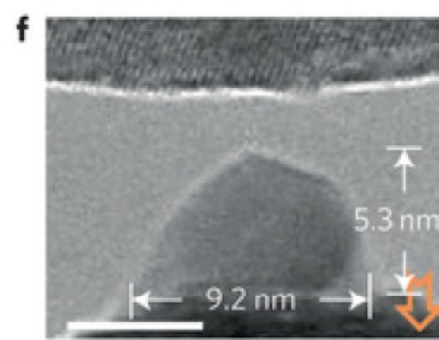

Fig. 21. Reversible pseudoelastic deformation of the Ag nanoparticles. (a-d) The Ag nanocrystal underwent a compression and (e, f) stretching cycle, almost recovered its initial size (a). Scale bars, $5 \mathrm{~nm}$. The orange arrows indicate the movement direction of the W tip. Reprinted with permission from Henry CR. 2014. Metal nanocrystals deforming like liquid droplets. Nature Materials 13, [232]: @2014 Nature Publishing Group.

ticle size, which has been proposed to be between 10 and 30 nanometers according to computer simulations and transmission electron microscopy analysis [233], on a recent atomistic simulation study by Li [234], it is shown that under very high stresses, dislocations can be accelerated to approach the speed of shear wave over a distance as short as $10 \mathrm{~nm}$ and such high speed dislocations often react in counter-intuitive manners that are beyond the descriptions of conventional dislocation behavior. As they described a high-speed dislocation can "rebound" when hitting a free surface rather than annihilate. When two high-speed dislocations collide, they can "penetrate" through each other. An individual dislocation can even spontaneously generate multiple dislocations via self-dissociation. These anomalous mechanisms lead to rapid proliferation of dislocations that are strongly correlated both spatially and temporally, and as such may play a role in high-stress and high-strain-rate plastic deformation.

Employing a collection of algorithms that assess the mechanical behavior of two-dimensional nanopillars (referred as nanoslab), Yan and Sharma [235] carried out a study of the mechanical compression behavior of nanoslabs under both low and high strain rates ( 1 and $10^{8} \mathrm{~s}^{-1}$ ). Their developed model was a 116 atom $\mathrm{Ni}$ nanoslab with the $x-y$ dimensions of $25 \times 70 \AA$, periodic in the $z$-direction. According to their results, while high-strain rate deformation proceeded with the appearance of shear band, the partially rotation of crystal orientation, and a barrel-shaped structure, the slow-strain rate results exhibited a dramatically different behavior and deformation proceeded with the start of amorphization from the top and bottom surfaces and was followed by material extrusion from the free surfaces. However, the central region of the nanoslab remained crystalline (Fig. 22). As they concluded the response of nanostructures to slow compression is more "liquid-like" and accompanied by extensive surface reconstructions which is in consistent with Sun et al. [233] results.

\subsubsection{Effect of pre-strain and aspect ratio}

Schneider et al. [236] performed micro-compression tests on pre-strained nickel single crystals to investigate the effect of the initial dislocation arrangement on the behavior and size dependence of small scale metal structures. They studied $5 \%$ and $20 \%$ pre-strained Ni pillars with different sizes, ranging from $200 \mathrm{~nm}$ to $5 \mu \mathrm{m}$. They found that $20 \%$ prestrained pillar's exhibited similar shapes for the stress-strain curves, with larger and less frequent strain bursts curves, comparing the $5 \%$ pre-strained pillars. However, the size dependence of the yield strength and strain hardening behavior was different than those of the $5 \%$ pre-strained pillars and for small pillar diameters the hardening was more pronounced for the $5 \%$ pre-strained sample (as quantified in Fig. 


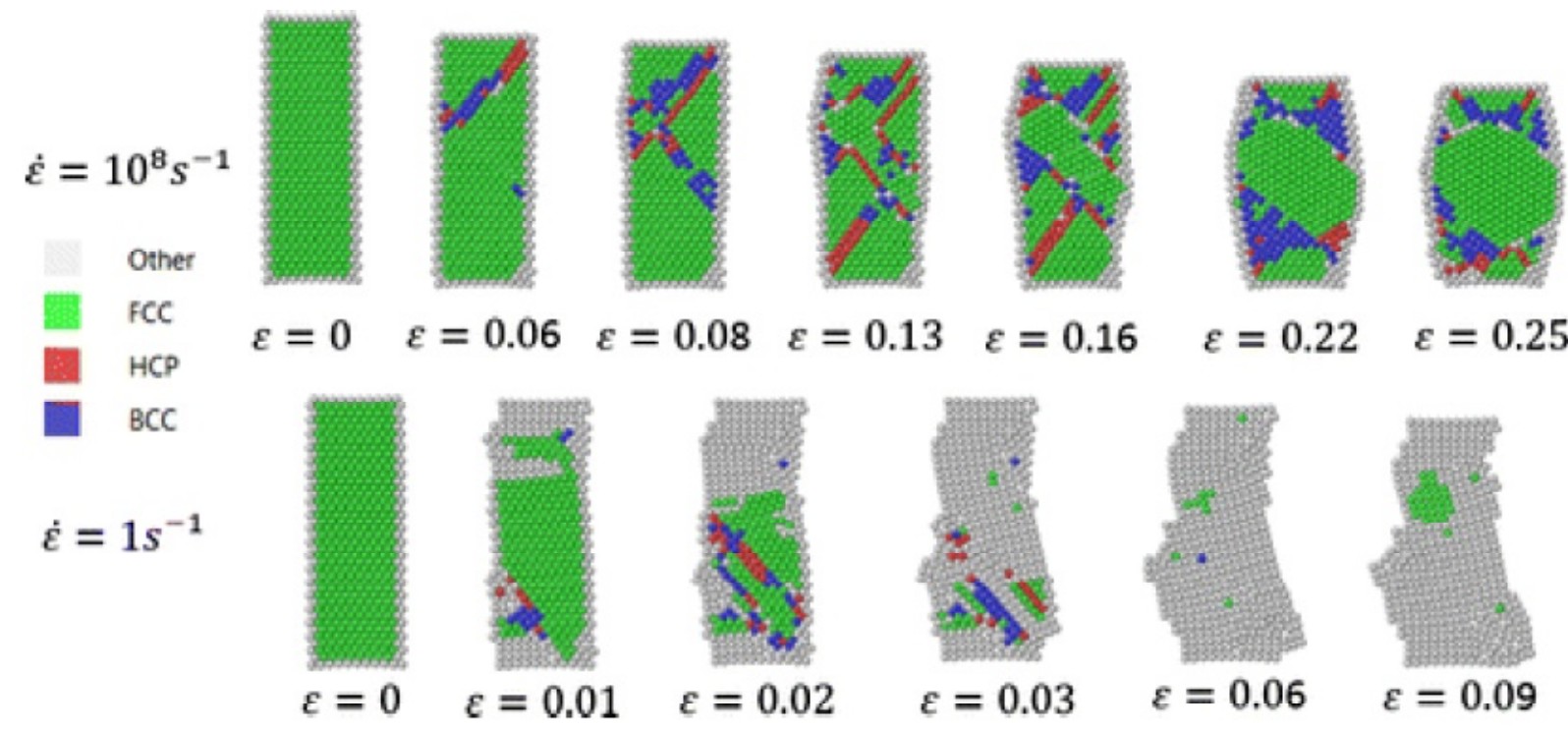

Fig. 22. Structure evolution of Ni nanoslab under compression at high strain rate (top row) and low strain rate (bottom row). For the case of high strain rate deformation, the structure experiences the appearance of shear band, the partially rotation of crystal orientation, and a barrel-shaped structure. For the low strain rate deformation, amorphization starts from the top and bottom surfaces and is followed by material extrusion from the free surfaces. Reprinted with permission from Yan X, Sharma P. 2016. Time-Scaling in Atomistics and the Rate-Dependent Mechanical Behavior of Nanostructures. Nano Letters 16, [235]: @ 2016 ACS Publications.

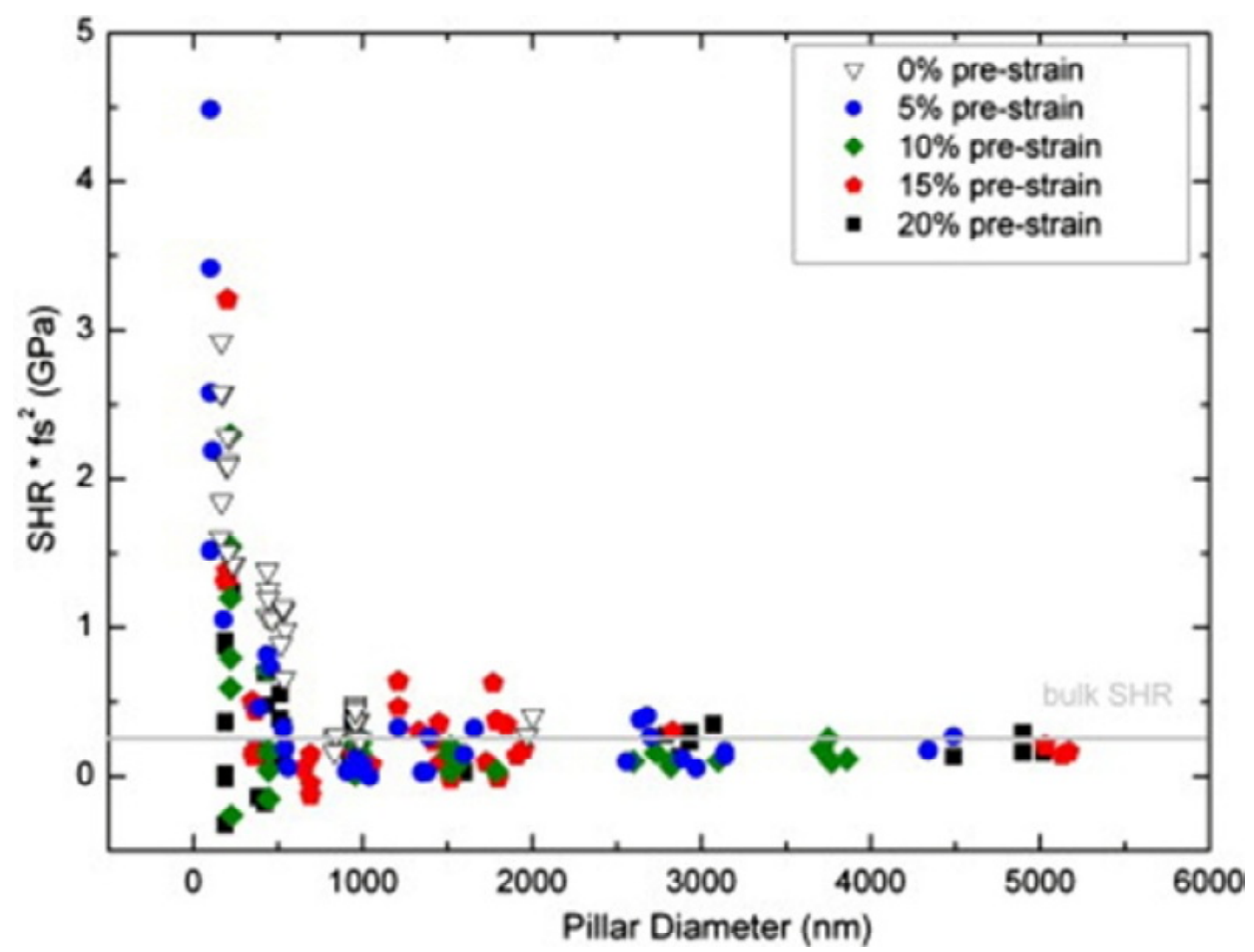

Fig. 23. Strain hardening rate (SHR) versus pillar diameter for pre-strained Ni single crystals. In order to take into account the sample orientations, the SHR was multiplied by Schmid factor square $\left(\mathrm{fs}^{2}\right)$. Reprinted with permission from Schneider AS, Kiener D, Yakacki CM, Maier HJ, Gruber PA, et al. 2013. Influence of bulk pre-straining on the size effect in nickel compression pillars. Materials Science and Engineering: A 559, [236]: (c) 2013 Elsevier Ltd. 
23). Analysis of TEM images showed that the dislocation density remained in the same order of magnitude for $5 \%$ and $20 \%$ pre-strained sample deformation, although the cell wall dislocation density was higher for the $20 \%$ pre-strained specimens. As Schneider at al. [236] stated, neither dislocation starvation nor mechanical annealing were explicitly observed with increasing dislocation density by prestraining and as the pillar size decreases from the micron into nano, a size dependent mechanism begins to dominate, independent of dislocation density. In situ TEM observations showed that dislocations were nucleated near the surface for the sub $200 \mathrm{~nm}$ diameter pillar, regardless of the presence of dislocations within the pillar.

Milne et al. [237], observed a distinct change in the deformation mode occurs as the aspect ratio of the Al pillar was increased. As they observed, the dominant deformation mode in 2:1 aspect ratio 900 $\mathrm{nm}$ diameter pillars was collective slip on multiple slip systems. A reduction of the pillar diameter with associated increased aspect ratio $(4: 1$ for $450 \mathrm{~nm}$ diameter) leaded to significant delocalized deformation and bulging at the apex to relieve stress. Apex work hardening was followed by slip band formation on a secondary slip system further down the pillar. A transition to a new mode of deformation was observed for nanopillars with diameters smaller than $250 \mathrm{~nm}$ and aspect ratio of $6: 1$. Axial buckling of a high aspect ratio pillar under axial compression, resulted to the lateral shear of the pillar apex and generating a bent morphology and a $90^{\circ}$ kink with further loading.

\subsection{BCC metals}

Among the common crystal structures of metals, body centered cubic (bcc) structures pose different conditions for plasticity compared with the fcc ones, due to the higher Peierls stress and easier crossslip of the slower screw dislocations. The deformation of bcc metals is generally governed by the screw dislocations, whose mobility is signiûcantly lower than that of the edge components, and the possibility of cross slip mechanism increases the probability of forming dislocation networks which results in the creation of immobile dislocation sources inside the nanopillar [57].

Although mechanical annealing has been widely observed in fcc metals, many scholars believe that such phenomena cannot exist in bcc metals for the following reasons. For the first reason, in a molecular dynamics simulation on Mo nanopillar deformation, Weinberger and Cai [39] found that under com- bined effects of the image stress and dislocation core structure, a dislocation nucleated from the surface of a BCC pillar generates one or more dislocations moving in the opposite direction before it exits from the surface. According to their results, the process of self-multiplication is repeatable so that a single nucleation event is able to produce a much larger amount of plastic deformation than that in FCC pillars. As the second reason, bcc metals are less size dependent at room temperature than fcc small samples $[30,40,41,55,164]$. As the third reason, postmortem STEM micrographs observation found that deformed, nano-scaled, single crystal Iron still have obvious dislocations in the crystal [238]. However, Huang et al. [239] showed that significant mechanical annealing occurs in BCC Mo pillars, when their diameters decrease to hundreds of nanometers.

Therefore, the governing mechanism for deformation of bcc nanopillars may be different from that of fcc nanopillars, and many scholars have tried to explain the size dependency of BCC nanopillar behaviour. Recently, pillar compression studies of different bcc metals including Mo $[30,41,48,55$, 94,164,240-242], Nb [48,49,55,95], Ta [48,55,58], $\mathrm{V}[56,243]$, W $[48,55,58,95]$, and Fe $[238,244,245]$, have been reported.

Fig. 24 shows typical compressive stress-strain curves of [235] oriented Mo, W, and Nb pillars with various diameters in compression, showing much higher attained flow stresses by the nanopillars with smaller diameters. It is noteworthy that the shapes of these curves demonstrate that the plastic deformation regions for smaller pillars are composed of discrete strain bursts followed by reloading segments. Most of the reloading segments appear to be elastic as is observed in fcc metals.

\subsubsection{Dislocation networks formation}

Greer et al. [30], compared the deformation behavior of fcc and bcc single crystals and reported that the dislocation starvation model may not apply to b.c.c. pillars at the diameters considered in their study $(300-800 \mathrm{~nm})$. As they described, the dislocation residence time inside the bcc crystal is relatively longer than that of fcc pillars, and the image stresses seem to enhance a dislocation's afûnity for self-replication when approaches a free surface and entanglement of these dislocation segments inside the pillar can contribute to increased ûow stress, similar to the forest hardening model that describes the plasticity of bulk crystals (Fig. 25). 

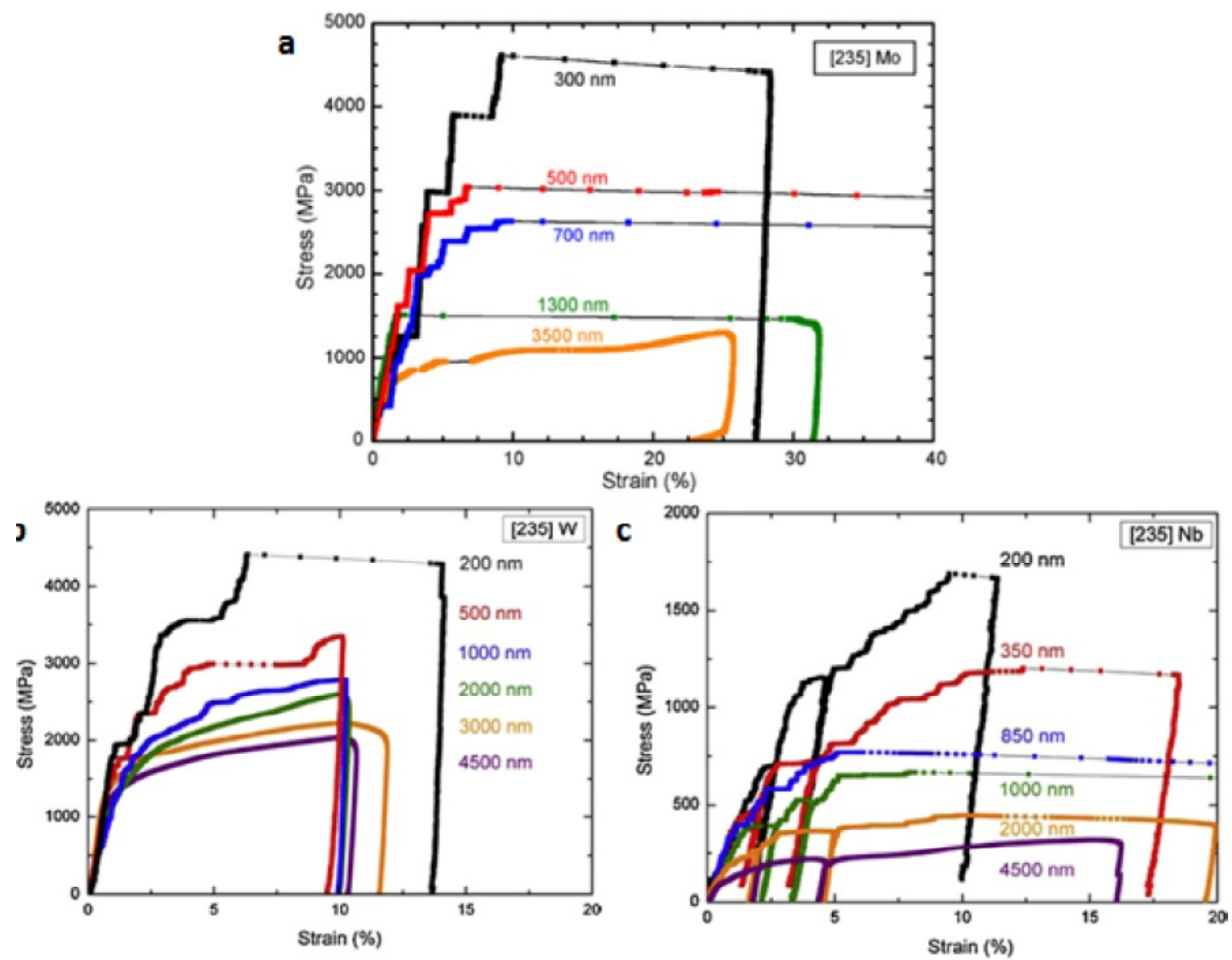

Fig. 24. Typical compressive stress-strain curves of [235] oriented (a) Mo, (b) W (c) Nb pillars [164,166]. Reprinted with permission from Schneider AS, Clark BG, Frick CP, Gruber PA, Arzt E. 2009. Effect of orientation and loading rate on compression behavior of small-scale Mo pillars. Materials Science and Engineering a-Structural Materials Properties Microstructure and Processing 508 [166]: (c) 2009 Elsevier Ltd. Reprinted with permission from Schneider AS, Frick CP, Clark BG, Gruber PA, Arzt E. 2011. Influence of orientation on the size effect in bcc pillars with different critical temperatures. Materials Science and Engineering a-Structural Materials Properties Microstructure and Processing 528 [168]. (c) 2011 Elsevier Ltd.
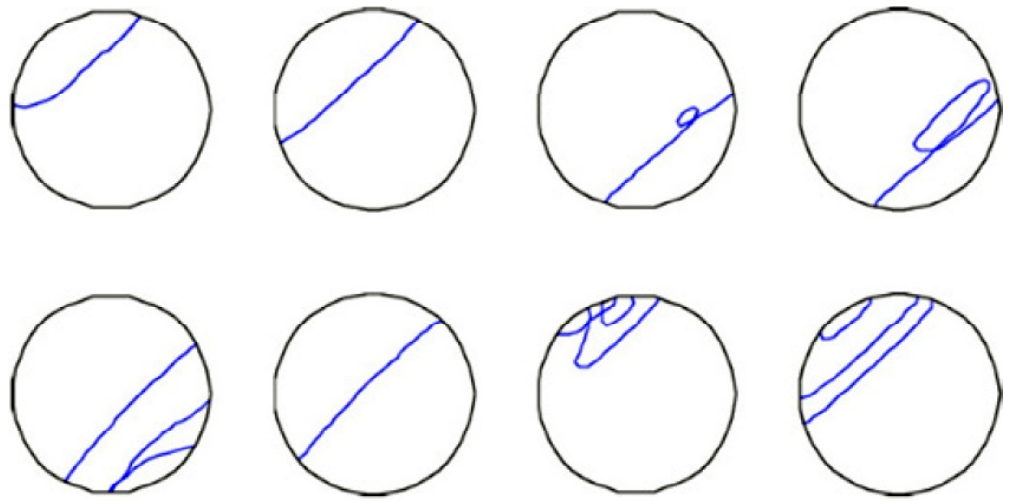

Fig. 25. Dislocation gliding along $(0 \overline{1} 1)$ plane in a b.c.c. pillar. The dislocation orients as a screw one and emits additional dislocations promoting strain hardening. Reprinted with permission from Greer JR, Weinberger CR, Cai W. 2008. Comparing the strength of f.c.c. and b.c.c. sub-micrometer pillars: Compression experiments and dislocation dynamics simulations. Materials Science and Engineering a-Structural Materials Properties Microstructure and Processing 493, [30]: (c) 2008 Elsevier Ltd. 

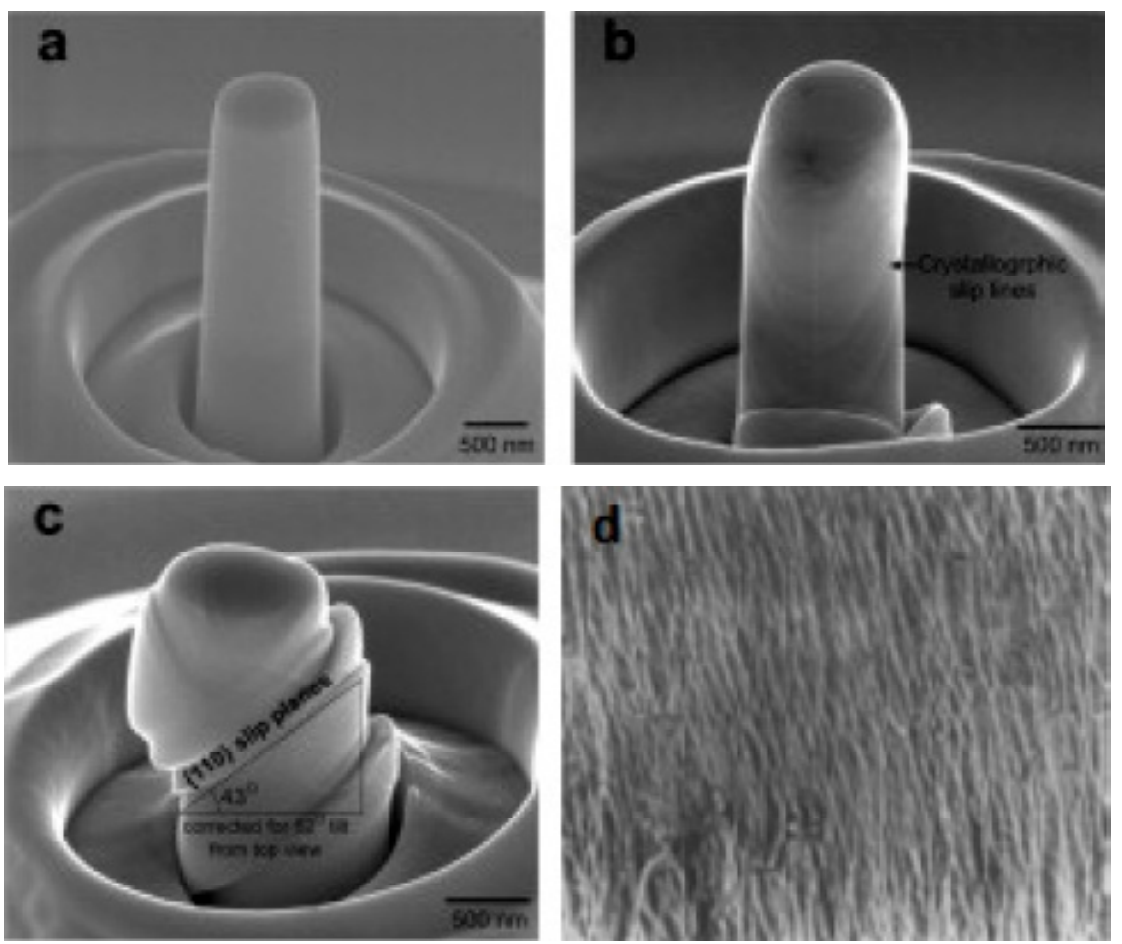

Fig. 26. SEM images of (a) nanopillar before compression, (b and c) severely compressed (up to $~ 30 \%$ true strain) nanopillars including pronounced crystallographic slip lines [49] d) Wavy lines in (100) surface of bulk $\mathrm{Nb}$ single crystal after $42 \%$ shear strain in compression, in which traces of both active slip planes are parallel [247]. Reprinted with permission from Kim JY, Jang DC, Greer JR. 2009. Insight into the deformation behavior of niobium single crystals under uniaxial compression and tension at the nanoscale. Scripta Materialia 61, [49]: (c) 2009 Elsevier Ltd.

As Kim et al. [48] pointed out, while FIB-fabricated bcc Mo nanopillars show size effects in the flow stress, the underlying foundation for this size dependent strength is quite the opposite of fcc metals and rather than annihilating at the free surfaces, dislocations in bcc metals have been found to form intricate networks as a result of mechanical deformation even in the smallest pillar size of $\sim 200 \mathrm{~nm}$ diameter [48].

SEM images of niobium $(\mathrm{Nb})$ single crystalline nanopillars before and after uniaxial compression are shown in Fig. 26. The compressed nanopillars showed fine and coarse parallel slip lines at the surface (Figs. 26b and 26c). The measured angles between most of the slip planes and the $<001>$ loading direction, $\left(\sim 43^{\circ}\right)$, shows that many slip planes are likely to be in $\{110\}$ planes. Generally, dislocation motion via cross-slip is prevalent in bcc metals since the screw dislocations are able to glide in different $\{110\}$ planes or combinations of $\{110\}$ and $\{112\}$ planes, often resulting in wavy and ill-defined slip lines [246]. However, Kim et al. [49] deformed nanopillar images, revealed that the slip traces at the surface are not wavy, but rather are elliptical, indicating the preferential confinement of the dislo- cation loops to a single slip plane without crossslipping. This observation is different from the observed wavy slip traces in the single crystalline bulk $\mathrm{Nb}$ samples after compression (Fig. 26d, [247]), showing that the mean free path of a dislocation loop's travel prior to cross-slip in Nb is larger than micrometer. This is probably due to the non-negligible effects of the image force imposed by the free surface.

Fig. 27 shows the bright-field TEM images and the corresponding diffraction patterns of the $\mathrm{Nb} 100$ $\mathrm{nm}$ diameter pillar before and after compression [49]. Besides the small FIB damage dislocation loops, no obvious dislocation lines are present in the pillar and since the dislocation density of a well-annealed metal is known to be on the order of $10^{8}-10^{12} \mathrm{~m}^{-2}$, it could be concluded that there are no or rare dislocations in a $100 \mathrm{~nm}$ pillar. The deformed image, however, clearly represents the formation of a complex dislocation network, display by the numerous black features present throughout the pillar, which have evolved in result of compression. An interesting point is that, the dislocation line segments in crystallographic planes are short, parallel to each another, and straight, indicating that dislocation ava- 

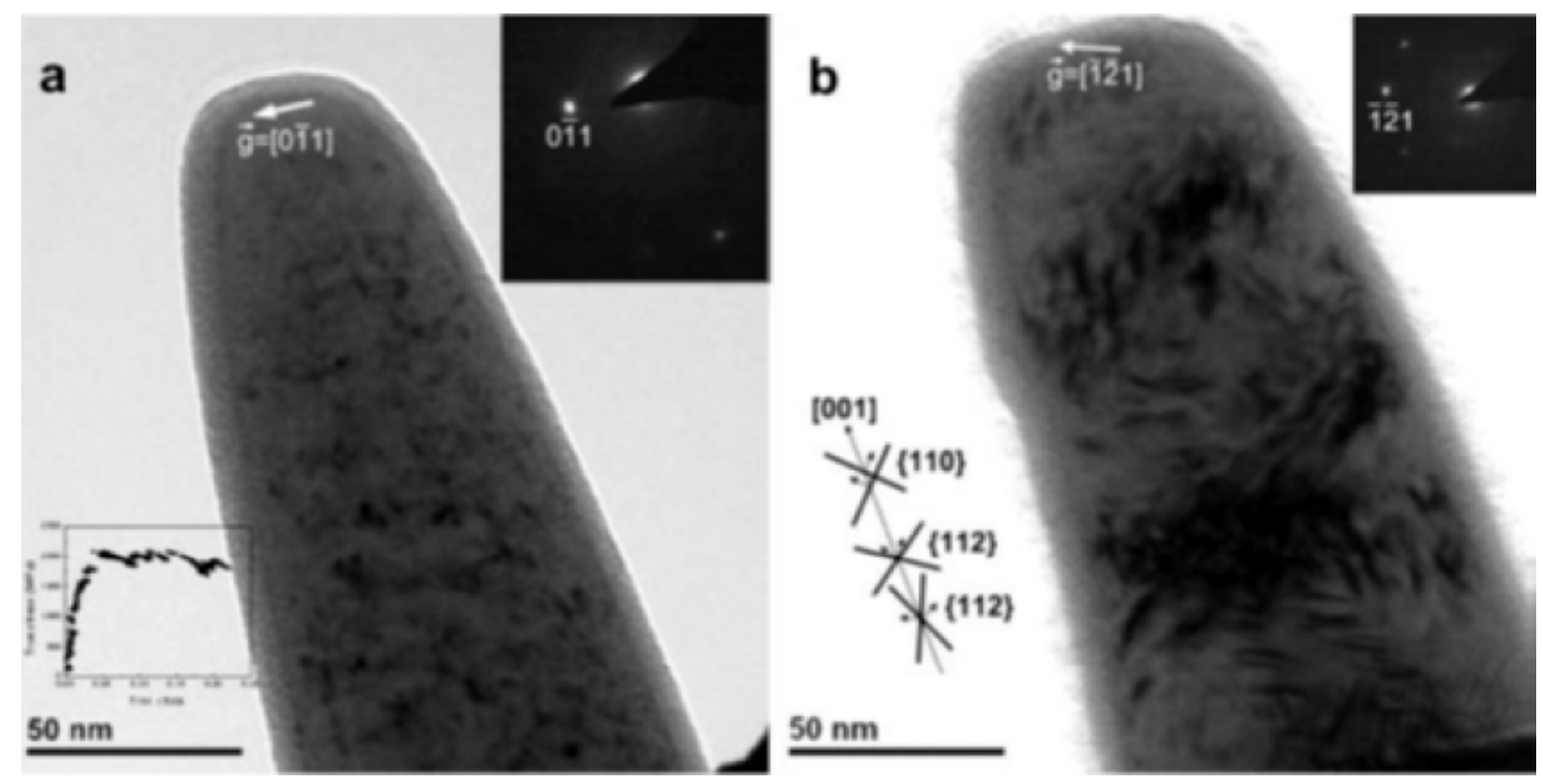

Fig. 27. Bright-field TEM images and corresponding diffraction patterns of $100 \mathrm{~nm} \mathrm{Nb}$ nanopillar (a) before and (b) after compression, in which the true stress-strain curve and representative slip planes are inserted. Reprinted with permission from Kim JY, Jang DC, Greer JR. 2009. Insight into the deformation behavior of niobium single crystals under uniaxial compression and tension at the nanoscale. Scripta Materialia 61, [49]: (c) 2009 Elsevier Ltd.

lanches probably occurred in parallel planes without interacting with one another. Moreover, no pinning points or internal Frank-Read sources can be seen in the image, which confirms the lack of dislocation cross-slip [49].

\subsubsection{Dislocation starvation}

Fig. 28a shows the stress-strain curve of bcc single crystal $\mathrm{Fe}-3 \% \mathrm{Si}$ pillar in result of compression [245]. As is observed the pillar undergoes a series of pronounced pop-ins which are indicated by numbers, and the curve is separated into three parts based on the stress level and dislocation dynamics. Figs. 28b-28e show snapshots of the No. 6 popin event occurred in part II. An edge dislocation remained at the bottom of the pillar before the No. 6 pop-in event, and is temporarily sessile, as marked. Fig. 28b shows the moment immediately before the pop-in event. As seen several sets of dislocations formed and escaped the pillar during the No. 6 popin event. Two sets of them are illustrated in Figs. 28c and 28d. In Fig. 28e, the formed dislocation No. 5 remained at the bottom of the pillar, which might be associated with the unexpected taper shape of the sample. Pop-in events Nos. 4 and 5 are similar to pop-in event No. 6 , which is the edge dislocation dominated process. Figs. 28f-28i presents snapshots of pop-in event No. 9, which occurred in zone III. As Zhang et al. [245] described, the pop-in events in zone III are screw dislocation dominated and are completely different from those of zone II. The formed dislocations (1-3) gradually moved toward the pillar surface during the deformation. Even though parts of the newly formed screw dislocations are covered by the carbon layer, it can be seen that all of the screw dislocations which move in the same direction (from the bottom to the top) do not undergo self-multiplication as described in Refs. [30,39], since self-multiplication involves the formation of a new dislocation moving in the opposite direction to the original ones. It is interesting to note that, the amounts of stress needed to activate edge (part II) and screw dislocations (part III) are different. This might be related to the difference in Peierls barrier stress between edge and screw dislocations in bcc crystallite which originates from the dislocation core structure [248].

Fig. 29 compares the residual dislocation structure in the deformed 1000 and $200 \mathrm{~nm}$ iron pillars [238]. As it is observed in the STEM images, the residual dislocation structure in the $1000 \mathrm{~nm}$ pillars is quite different from that of $200 \mathrm{~nm}$ samples. For $1000 \mathrm{~nm}$ pillar (Fig. 29a), a high density of dislocations was found in the highly deformed zone while only a few dislocations are present in other areas. 

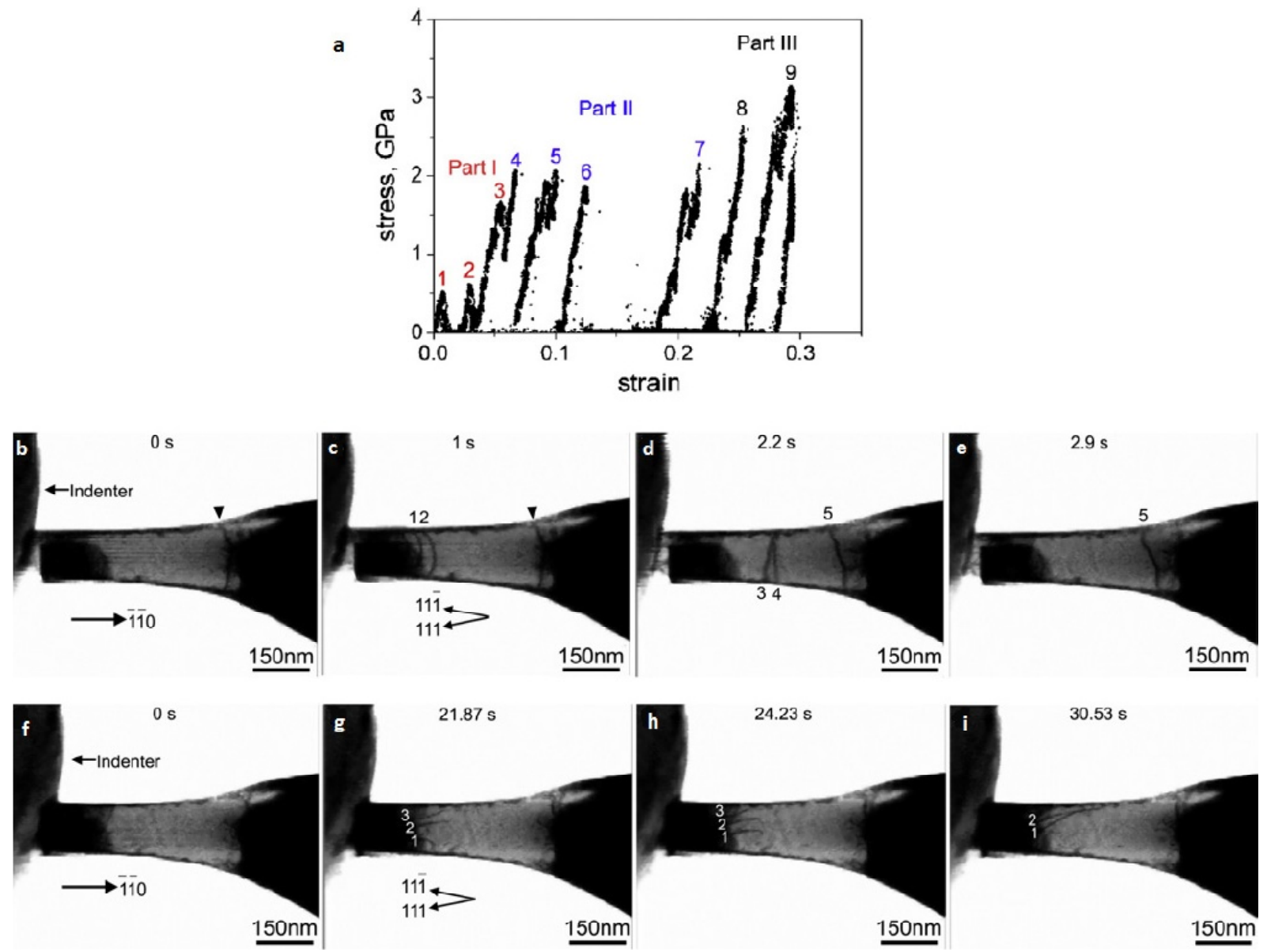

Fig. 28. (a) resulted stress-strain curve of $\mathrm{Fe}-3 \% \mathrm{Si}$ single crystal pillar compression, the numbers indicate the pop-in events and the curve is divided into three parts. (b-e) Snapshots of the No. 6 pop-in event that occurred in part II; (b) The moment before the pop-in event. A temporarily sessile edge dislocation remained at the bottom of the sample; (c) dislocations 1 and 2 formed at $1 \mathrm{~s}$ and escaped the pillar within= $0.03 \mathrm{~s}$; (d) another set of dislocations (3-5) formed at $2.2 \mathrm{~s}$; (e) dislocations 3 and 4 escaped the pillar. (fi) Snapshots of the No. 9 pop-in event that occurred in part III; (f) The moment before the pop-in event. (g-i) The dislocations moved from the lower pillar rim toward the upper pillar rim. Reprinted with permission from Zhang L, Ohmura T, Sekido K, Hara T, Nakajima K, Tsuzaki K. 2012. Dislocation character transition and related mechanical response in a body-centered cubic single crystal. Scripta Materialia 67, [245]. (c) 2012 Elsevier Ltd.

However, for the $200 \mathrm{~nm}$ pillar, only one residual dislocation can be found in the heavily deformed area, as indicated by the arrows. This is in consistent with dislocation escape due to the mechanical annealing phenomena.

Using in situ TEM compression of Mo nanopillars, Shan [105] demonstrated that with decreasing pillar diameter to hundreds of nanometers, significant mechanical annealing does occur in bcc Mo (Fig. 30a). In addition, there was a critical size ( $200 \mathrm{~nm}$ for Mo at room temperature) below which the strengthening exponent increased dramatically to that similar to fcc metals (Fig. 30b). As Shan [105] described, this regime of size effects in bcc con- verges to that of fcc, revealing deep connection in the dislocation dynamics of the two systems. Observed phenomena were attributed to the diminishing importance of lattice friction at high stresses, when the size enhanced flow stress exceeds a single screw dislocation's lattice friction.

\subsubsection{Cross-split of edge dislocations}

Kositski et al. [107], proposed a rapid conservative athermal dislocation mechanism by which edge dislocations overcome obstacles and change their glide plane by splitting (Fig. 31). This mechanism is a collective process by which edge dislocation splits 


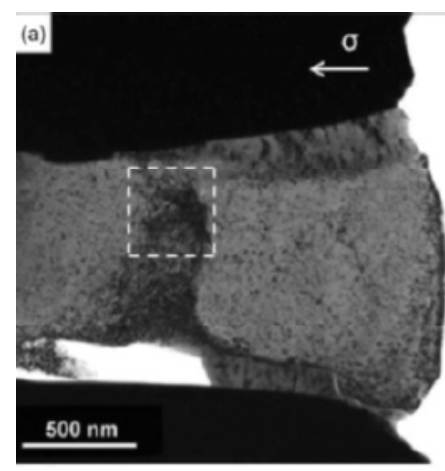

(c)

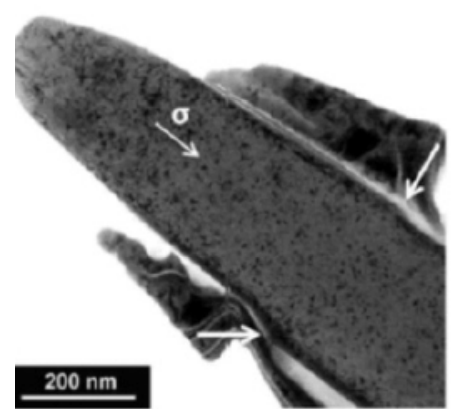

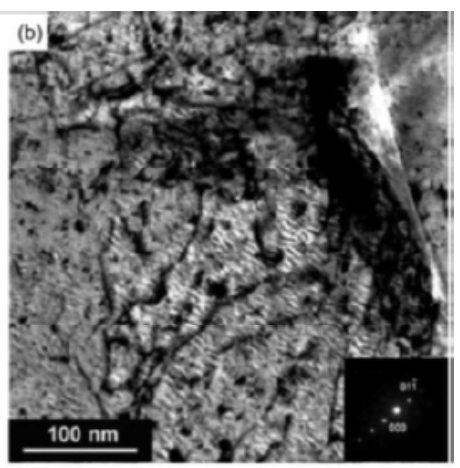

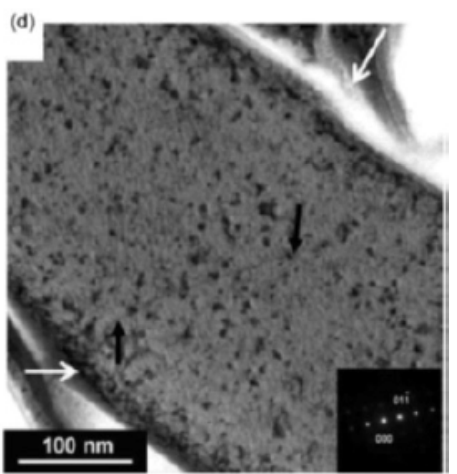

Fig. 29. Postmortem bright-field STEM images of the Fe pillars after compression tests. (a) $1000 \mathrm{~nm}$; (b) magnified image of the area framed in (a); (c) $200 \mathrm{~nm}$; (d) magnified image of the area indicated by the two white arrows in (c). The dislocation line marked by the black arrows. Reprinted with permission from Huang R, Li QJ, Wang ZJ, Huang L, Li J, et al. 2015. Flow Stress in Submicron BCC Iron Single Crystals: Samplesize-dependent Strain-rate Sensitivity and Rate-dependent Size Strengthening. Materials Research Letters 3, [238]: Copyright @ 2015 Informa UK Limited.
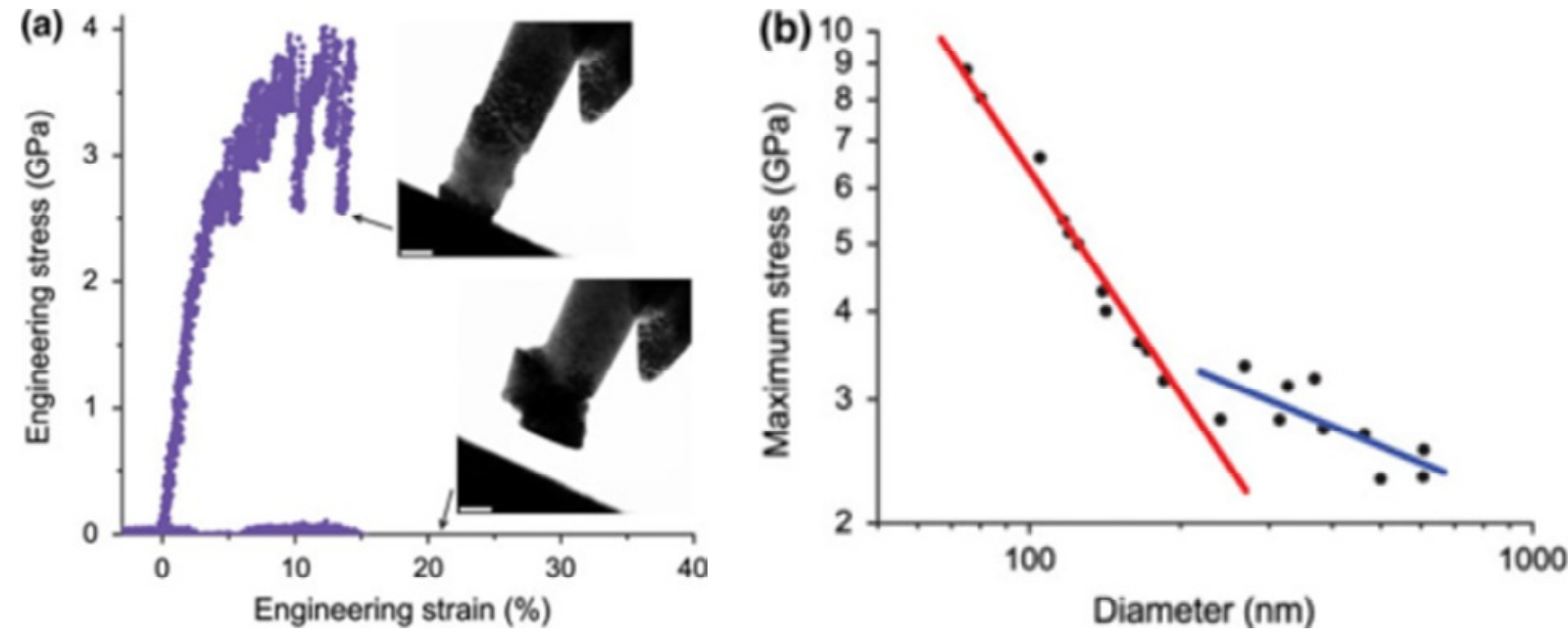

Fig. 30. (a) The typical engineering stress-strain curve for a $142 \mathrm{~nm}$ FIBed Mo single crystal. The insets are bright-ûeld TEM images taken before and after the dramatic strain burst. The scale bar represents $100 \mathrm{~nm}$. (b) Maximum ûow stress plotted as a double-log function of pillar diameter $D$, representing two different strengthening exponent values, 1.0 and 0.29 for red and blue lines, respectively. Reprinted with permission from Shan ZW. 2012. In Situ TEM Investigation of the Mechanical Behavior of Micronanoscaled Metal Pillars. JOM 64, [105]: @ 2012 Springer International Publishing AG.

into two edge dislocations which are not arrested by the obstacle. They performed MD simulations to gain insight into deformation of Fe nanoparticles. The simulations revealed that plasticity starts by nucleating dislocations at two opposite top vertices of the nanoparticle. Once nucleated, dislocations are curved with two ends on the facets of the nanoparticle, obtaining mixed characteristics at ends 
(a)
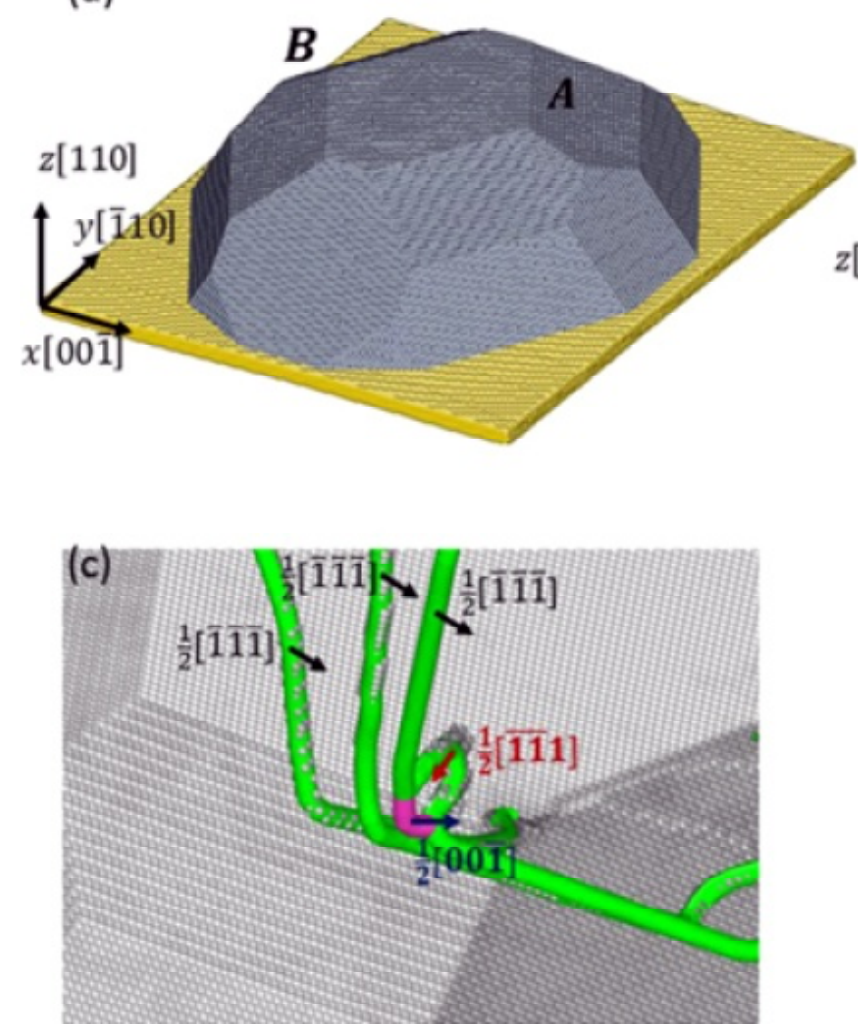

(b)

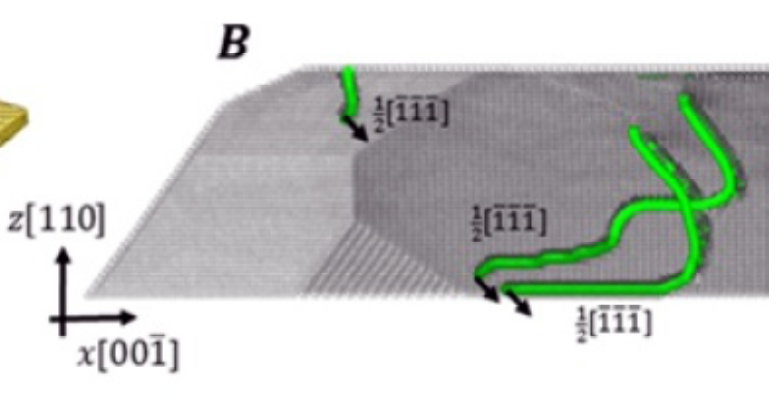

Fig. 31. MD simulation of a $20.8 \mathrm{~nm}$ high Fe nanoparticle under compression. (a) The two top vertices marked with "A" and "B" are the points where dislocations nucleate. (b) A three-dislocation pile-up formed at $B$ is shown (all the atoms were removed except those in the dislocation cores) (c) A view from the bottom on the dislocation pile-up shown in (b) slightly after the front $1 / 2[\overline{1} \overline{1} \overline{1}]$ dislocation in the pile-up splits into $[00 \overline{1}]$ and $1 / 2[\overline{1} \overline{1} 1]$ dislocations. (d) A bottom view into the particle after cross-split. The color represent height with red being the height of the top facet and blue the bottom. Reprinted with permission from Kositski R, Kovalenko O, Lee SW, Greer JR, Rabkin E, Mordehai D. 2016. Cross-Split of Dislocations: An Athermal and Rapid Plasticity Mechanism. Scientific Reports 6, [107]. @ 2016 Springer Nature.

and an edge characteristic at its center. As the applied stress increased, the dislocations move in the inward directions towards the substrate, and both ends traveled along the edges and the facets of the nanoparticles, straightening the overall dislocation line towards the edge character. In this mechanism, the dislocation line is finally arrested close to the substrate, where it gained an edge character along its entire line. If the particle is sufficiently wide, the slip planes of the dislocations nucleated at vertices $A$ and $B$ reach the substrate without interacting with one another. Since these dislocations are prevented from gliding by the interface, further deformation take place by additional nucleation events at the two vertices and inward propagation. These nucleation events do not produce catastrophic or substantial strain bursts as was observed in FCC nanoparticles [50] after each nucleation event and consecutive nucleation events require higher stress in order to overcome the back-stress from the growing pile up, which manifests itself in hardening of the nanoparticle as it is compressed [107].

\subsubsection{Effect of temperature}

Schneider et al. [166] proposed that the mobility of the screw dislocation controls the size effect of the bcc pillars and with decreasing $T_{c}$ (critical temperature; the temperature above which the flow stress becomes relatively insensitive to temperature and strain rate) or pillar diameter, the effect of relative screw dislocation immobility becomes less important and the strength of fcc and bcc pillars are determined by similar dislocation processes. Possible explanations for this behavior include kinetic pileups of screw dislocations [164] and kink nucleation at the pillar surface [39].

Generally, for bcc metals, when the temperature is at or above the $T_{c}$, the screw dislocations would glide much easier or the Peierls stress for 
bcc metals becomes effectively very small [249]. As Han et al. [57] pointed out, the size effects on the strength of bcc nanopillars are dependent on the $T_{c}$ and, equivalently, the Peierls stress (sp). When the testing temperature is higher than the critical temperature of a bcc metal, peierls stress can be neglected, and the strengthening mechanism is similar to that for fcc metals with the size effect exponent $\mathrm{n}$ in the range of 0.8-1.0. When $T<$ $T_{c}$, the effect of sp cannot be neglected, and $n$ would decrease with increasing $\mathrm{sp}$. A clear reduction in the dislocation density has been observed after deformation of $V$ nanopillars, suggesting a low $s p$ for $V$ at room temperature, as expected [57]. Thus some bcc metals like $\mathrm{V}$, display dislocation starvation mechanism due to their low Tc and low sp that facilitated dislocation motion and easier removal from the body of the nanopillar, although the rate of dislocation removal is slightly slower than that for fcc nanopillars [57].

Franke et al. [46] showed that there is a significant change in the plasticity of a high-melting point $\mathrm{BCC}$ material once the critical temperature is surpassed. As they described, the mean pop-in load increases from (111) to (110) and (100) which shows the highest resistance against localized yielding. The differences in the pop-in load of the different orientations was explained by the hydrostatic pressure presents in each orientation which aids nucleating defects such as twins and stacking fault (SF).

Abad et al. [58] studies on Ta and W micropillars showed that the strain hardening behavior is not only size dependent but, more importantly, test tempera-

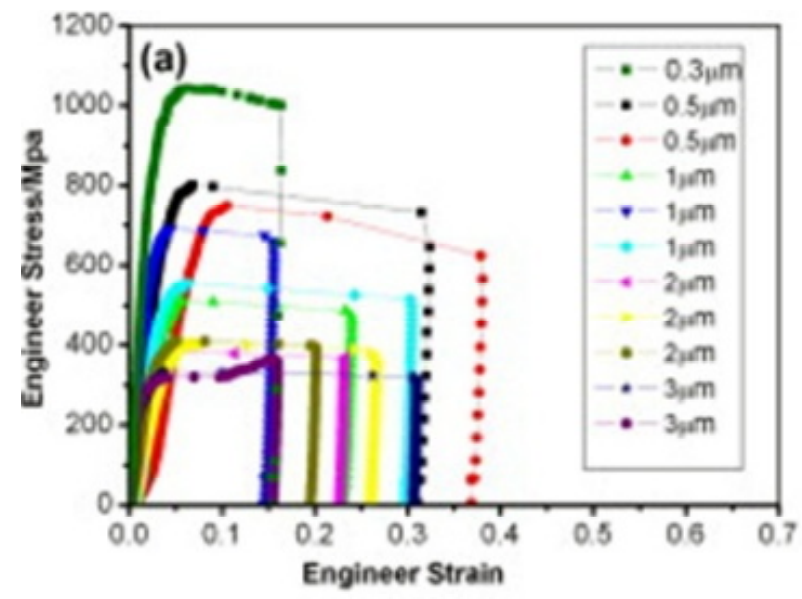

ture dependent and lower SHRs are observed at higher temperatures. This behavior is opposed to bulk BCC metals, where the easier mobility of screw dislocations at higher temperatures increases dislocation forest hardening and could be explained by the easier activation of dislocation sources near the surface and better dislocation mobility at higher temperatures.

\subsection{HCP metals}

Compared to bcc and fcc structures, metals with a hexagonal closed-packed (hcp) crystalline structure have smaller number of slip systems, which makes their plastic deformation more difûcult. For instance, both tension/compression twinning $[250,251]$ and various kinds of $<c+a>$ pyramidal slips [252] have been observed in bulk single or poly crystal hcp materials because of the limited number of <a> slips activated on the basal and prismatic planes. Especially for micro-compression under c-axis, twinning and $<\mathrm{c}+$ a $>$ pyramidal slips have been found to be the main sources of deformation $[61,62,253]$. According to Yu et al. [59] studies on titanium ally single crystal, the stress required for deformation twinning increases drastically with decreasing sample size until the sample size is reduced to one micrometer, below which the deformation twinning is entirely replaced by less correlated, ordinary dislocation plasticity. Meanwhile, compression results of pure magnesium single micro crystals showed no twinning in samples from approximately $2-10$ $\mu \mathrm{m}$ in diameter, and the pyramidal slips were found to dominate the plasticity $[61,62]$.

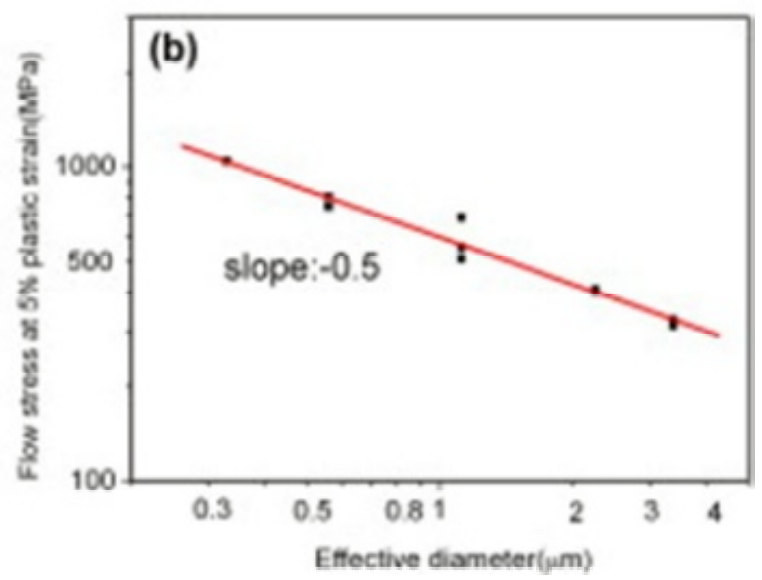

Fig. 32. Size effect in the flow stress of Ti pillars. (a) Engineering stress-strain curves of compressing Ti samples with different diameters; (b) The flow stress at $5 \%$ plastic strain vs the effective diameter (the equivalent circular pillar diameter of the square pillar; $d_{\text {effecive }}=\sqrt{4 S / \pi}$ ). Reprinted with permission from Sun QY, Guo Q, Yao X, Xiao L, Greer JR, Sun J. 2011. Size effects in strength and plasticity of singlecrystalline titanium micropillars with prismatic slip orientation. Scripta Materialia 65, [60]: @ 2011 Elsevier. 

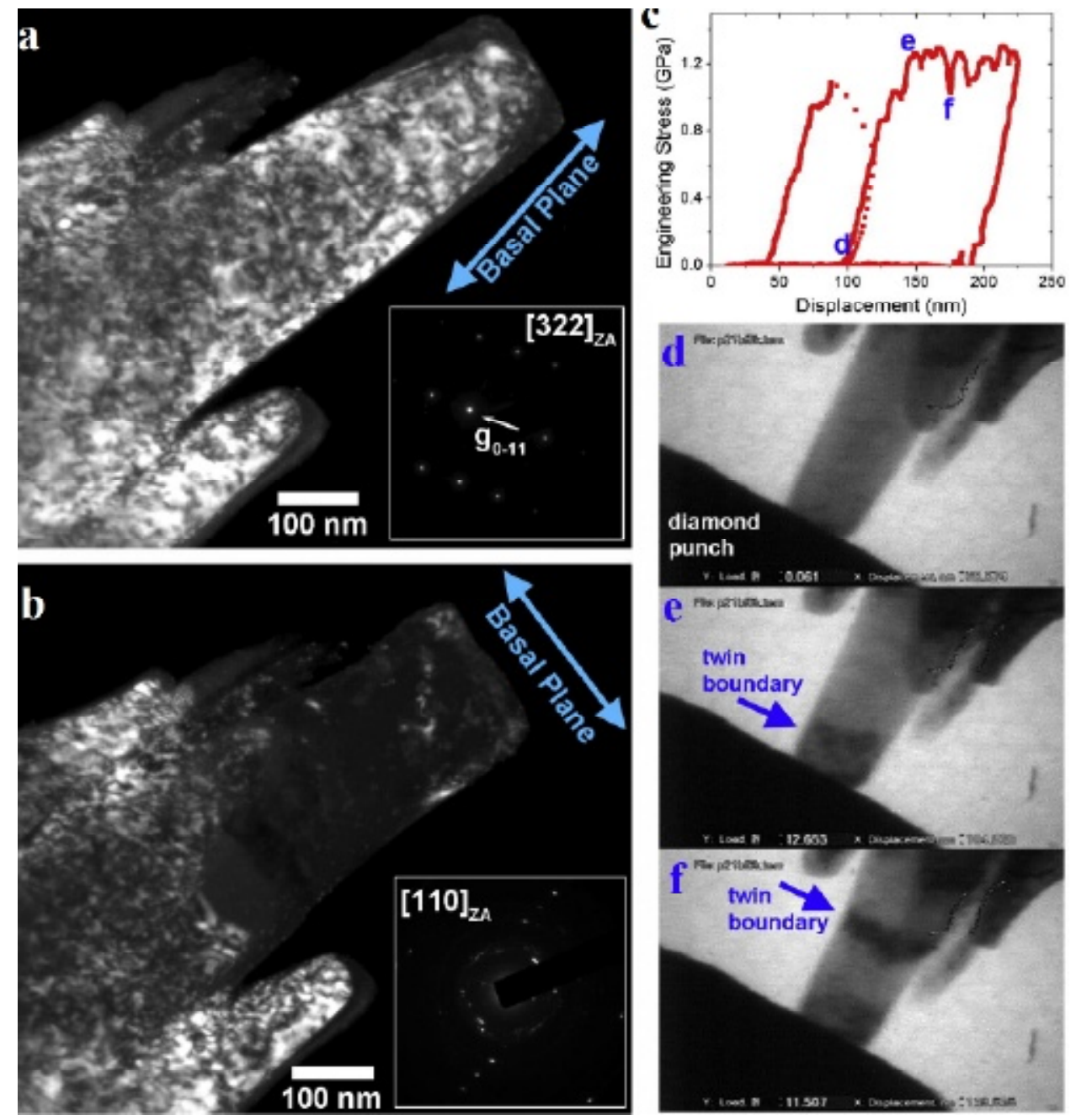

Fig. 33. Example of extension twinning in a $200 \mathrm{~nm}$ pure Mg pillar. (a) Dark-field image of the pillar before the test. (b) Dark-field image of the pillar after the test, showing that the pillar now has a different orientation from the base of the pillar. Inset shows the diffraction pattern of the twinned pillar, revealing that the basal plane is now roughly perpendicular to the loading axis. (c) Engineering stress- displacement curve of the pillar compression. The large load drop seen at $75 \mathrm{~nm}$ displacement is a result of a large dislocation burst through the pillar. (d-f) frames from the in situ video, corresponding to marked points in (c). At point (d) the pillar is relatively defect free (d) after the large burst of dislocations. At point (e) a twin is nucleated, with the boundary shown in (e). At point (f) the twin boundary has moved through the pillar and is seen in (f). The final result is a completely twinned pillar (b). Reprinted with permission from Ye J, Mishra RK, Sachdev AK, Minor AM. 2011. In situ TEM compression testing of Mg and Mg- 0.2 wt.\% Ce single crystals. Scripta Materialia 64, [63]. @ 2011 Elsevier.

Jin et al. [73] characterized the mechanical behaviors of electroplated sub-micron cadmium pillars by uniaxial compression techniques. Their results revealed that the mechanical strength of submicron cadmium structures is sensitive to strain rate and size dependent. As they observed while the strength of 0.5 and $1.1 \mu \mathrm{m}$ diameter specimens were slightly higher than bulk, the flow stresses of 130 $\mathrm{nm}$ diameter ones approached to the theoretical strength of cadmium. Representative compressive stress-strain curves of Ti pillars are shown in Fig. $32 a$ [60]. Clearly, the higher flow stress is observed in smaller samples, and the deformation is characterized by a single, large strain burst subsequent to yielding. Fig. 32b shows the size effect on the flow stress at $5 \%$ plastic strain $\left(\sigma_{f}\right)$, indicating Ti follows the power law rule (Section 3) with the exponent of -0.5 .

\subsubsection{Twining}

Ye et al. [63] performed in situ TEM nano-compression tests on around $200 \mathrm{~nm}$ diameter single crystal pure $\mathrm{Mg}$ and $\mathrm{Mg}-0.2$.wt.\% Ce pillars. They fo- 
cused on the two major deformation mechanisms in $\mathrm{Mg}$, i.e. basal plane sliding and twinning. A clear size effect was found for both deformation mechanisms, by measuring the critical stress for nucleation of either basal sliding or extension twinning. They also reported a significant reduction of twining critical stress in result of alloying with Ce. Fig. 33 shows formation of extension twinning in compression of a pure $\mathrm{Mg}$ single crystal [63]. The pillar started with a relatively high defect density caused by the FIB milling, as shown in Fig. 33a. During the first $50 \mathrm{~nm}$ of the compression the pillar went through the mechanical annealing process, whereby the mobile dislocations escaped from the pillar, leaving behind a rather clean pillar (Fig. 33d). The large drop in the stress showed by $d$ in Fig. 33c is the consequence of this event. The pillar has been then reloaded and when stress reached to approximately $1.26 \mathrm{GPa}$, a twin domain was nucleated from the tip of the pillar, as marked by the arrow on Fig. 33e. After the nucleation, the twin has grown towards the bottom of the pillar while the stress remained almost constant. Fig. 33f shows the growing twin domain with the boundary marked. The result is a new, almost perfect grain, with a boundary near the base of the pillar that is full of defects. The critical stress for twin nucleation in this case, demonstrating that there is also a size effect associated with the twining mechanism, as has been also observed in a Ti-Al alloy [59].
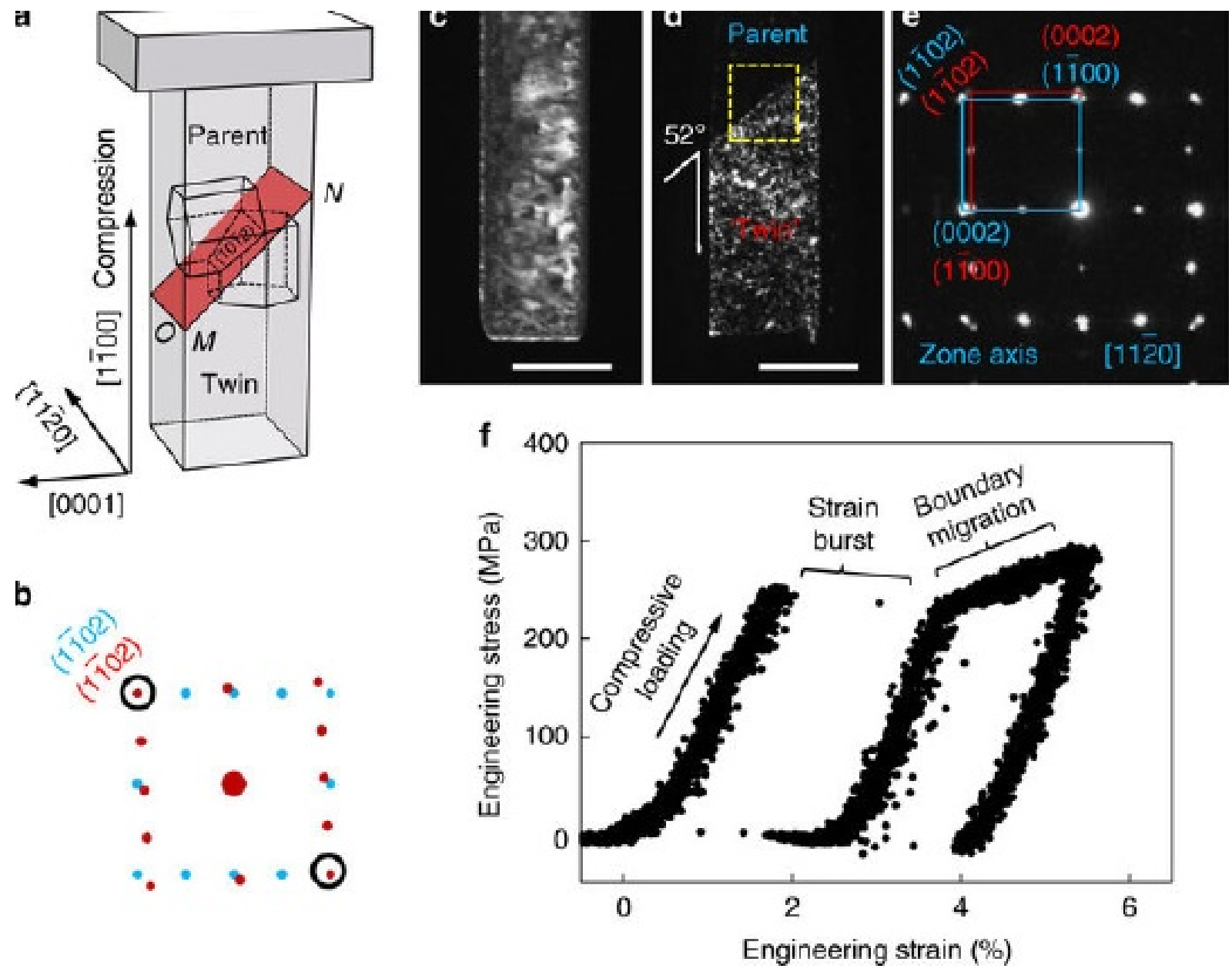

Fig. 34. In situ compression test on a submicron-sized single crystal Mg pillar.

(a) Schematic illustration of the crystal, loaded in [1 $1 \overline{1} 00]$ orientation. The $\{10 \overline{1} 2\}$ plane is highlighted in red.

(b) Selected area diffraction pattern (SADP) for an ideal $\{10 \overline{1} 2\}$ twin. (c,d) Dark-field TEM images of the pillar before and after compression. Scale bars, $400 \mathrm{~nm}$. (e) SADP taken from the framed area, demonstrating that $\{10 \overline{1} 2\}$ diffraction spots are separated instead of overlapping with each other. The zone axis is $[11 \overline{2} 0]$ for parent lattice. $(f)$ The corresponding stress-strain curve. Reprinted with permission from Liu BY, Wang J, Li B, Lu L, Zhang XY, et al. 2014. Twinning-like lattice reorientation without a crystallographic twinning plane. Nature Communications 5, [255]. ( 2014 Springer Nature. 

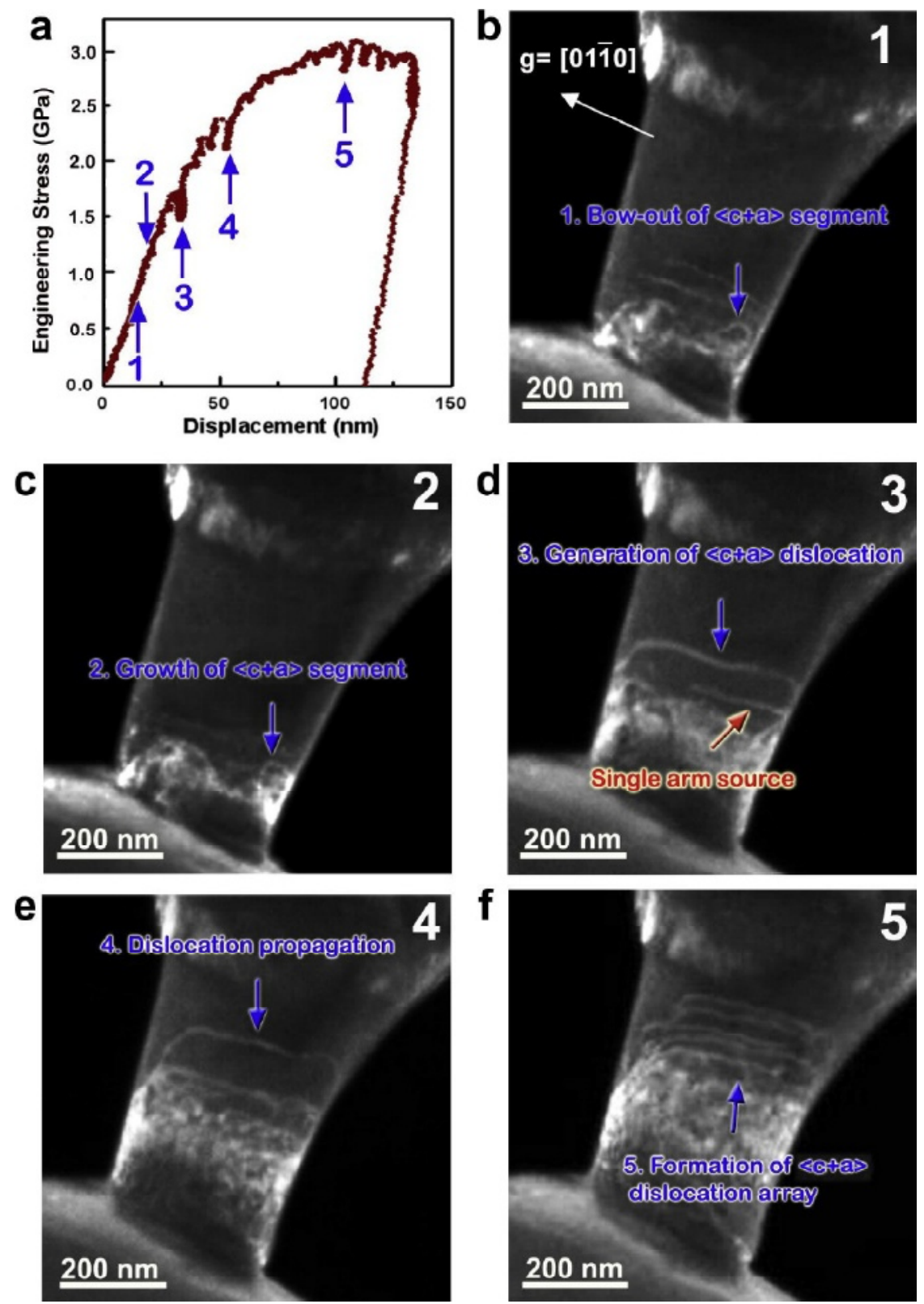

Fig. 35. (a) Engineering stress-displacement curve for nano-compression of $250 \mathrm{~nm}$ Ti-5Al pillar. (b and f) TEM images showing the formation and operation of $a<c+a>$ dislocation source and the corresponding mechanical response: (b) the bow-out of $a<c+a>$ segment, (c) the growth of the $<c+a>$ segment, (d) the generation of $\mathrm{a}<\mathrm{c}+\mathrm{a}>$ dislocation and (e) its propagation, (f) the formation of $\mathrm{a}<\mathrm{c}+\mathrm{a}>$ dislocation array. Reprinted with permission from Yu Q, Sun J, Morris JW, Minor AM. 2013. Source mechanism of non-basal $<c+$ a $>$ slip in Ti alloy. Scripta Materialia 69, [258]: @ 2013 Elsevier.

Yu et al. [254] further investigated the deformation twinning in pure Mg single crystal through different loading conditions. The formation of deformation twins was observed under different strain paths. As they found, high-density nanotwins can be "promoted" kinetically when high density of nucleation sites is correlated by the stress fields of twinning dislocations.

Carrying out the same experiments on pure magnesium single crystal, Liu et al. [255] reported the reorientation of the parent lattice to a 'twin' lattice, resulting in an orientational relationship akin to that of the conventional $\{10 \overline{1} 2\}$ twinning, but without a crystallographic mirror plane, resulting a plastic strain that is not simple shear. According to their observation, the boundary between the parent lattice and the 'twin' lattice is composed of semicoherent basal/prismatic interfaces instead of the $\{10 \overline{1} 2\}$ twinning plane and migration of this boundary is dominated by the movement of these inter- 
faces undergoing basal/prismatic transformation via local rearrangements of atoms (Fig. 34).

\subsubsection{Dislocation slip}

Fan and El-Awady [256] investigated deformation modes in Mg nanocrystals during uniaxial tension, uniaxial compression, and pure bending using molecular dynamics simulations at room temperature. Their results for compression loading showed that yielding is dominated by only dislocation slip on the pyramidal $(\theta<15 \mathrm{deg})$, basal ( $15 \mathrm{deg}<\theta<60$ $\mathrm{deg})$ and prismatic $(\theta>60 \mathrm{deg})$ planes and nucleation stress in general decreased with increasing $\theta$ for both uniaxial tension and uniaxial compression loadings. Electron backscattered diffraction and microstructural analyses of the deformed micrometer-sized Mg single crystals samples [257] revealed that the plastic deformation in basal slip oriented crystals occurs only by slip while twin oriented crystals deform by both slip and twinning modes. The twin oriented crystals exhibit a higher strain hardening during plastic deformation comparing to the single slip oriented crystals.

In situ TEM nano-compression test containing the formation of $a<c+a>$ dislocation in a $250 \mathrm{~nm}$ Ti-5AI FIB milled pillar is shown in Fig. 35, [258]. Once the load was applied, the dislocations were emitted from the contact surface. The newly generated dislocations were mainly determined to be $<a>$ type. The pre-existing $<c+a>$ dislocations are stable and have not shown any detectable activity at the beginning of the test. Since the resolved shear stress on $<a>$ dislocations is almost zero in this orientation, the newly generated $<a>$ dislocations were essentially immobile and quickly became entangled, resulting in a high-density dislocation structure. As the load increases, some dislocation segments bowed out from this entanglement (Fig. 35b). The bowing indicates a varying line direction within the single crystal that is only compatible with the $<c+a>$ dislocation type. This segment continued to grow, as shown in Fig. 35c. The dislocation activities are localized in the top of the sample and the stress increased almost linearly until it reached $\sim 1.7 \mathrm{GPa}$ (point 3 in Fig. 35a). Beyond this point, a load drop is seen. Accordingly, a single arm source was observed as marked by the red arrow in Fig. $35 \mathrm{~d}$, as well as a few long dislocations. As the emission of the glissile $<c+a>$ dislocations continues, the stress vs. displacement curve deviates from near linearity and demonstrates work hardening. When the stress on the sample reaches $\sim 2.34 \mathrm{GPa}$, a second load drop can be seen in the curve corre- sponding to another emission event of $<c+a>$ dislocations (Fig. 35e). The stress at the contact surface eventually reaches $\sim 3.2 \mathrm{GPa}$. Several significant stress drops occur near this maximum, and the popping out of $<c+a>$ dislocations was observed successively (shown in Fig. 35f), resulting in an array of $<c+a>$ dislocations. In this specific case, the pre-existing $<c+a>$ dislocations in Fig. $35 b$ stayed immobile until the very end, probably due to the strong pinning force due to the high-density dislocation structure. Yu et al. [258] also pointed out that, in some other cases, the $<c+a>$ dislocations moved through a depinning process instead of operating as a dislocation source.

Tang et al. [259] investigated the specific slip mechanisms on $\{10 \overline{1} 1\}$ and $\{11 \overline{2} 2\}$ pyramidal planes of an hcp magnesium single crystal. Their results implied that the slip on $\{10 \overline{1} 1\}$ plane is more likely to nucleate with a relatively easy dissociation type comparing to the one on $\{11 \overline{2} 2\}$ plane and no twinning was found under $c$-axis compression by examining the stepwise movement of atoms involved, fully supporting the experimental observations of micro-compression and the theoretical analysis on twinning formation $[61,62]$.

The strong size effect on deformation twinning in hcp metals can be correlated with the availability of dislocation sources which are less in small samples. In the "stimulated slip" model proposed by Yu et al. [59] the formation of a deformation twinning is accomplished by the stimulated slip plane by plane, and the stimulated slip between two planes is catalyzed by promoter defects, such as screw dislocation poles. Since the surface in single crystal materials will determine the confined volume to restrict the number of dislocations, it is believed that the number of available "promoters" is less in smaller volumes. Therefore, the smaller the dimension of the sample, the less possibility of coupling two adjacent slip planes by threading screw pole dislocations, and thus less probability of having deformation twinning mechanism. The disappearance of twins in sub-micron and micro-sized samples of $\mathrm{Mg}$ and Ti alloy confirm this theory $[61,62,64]$. Guo et al. [260] investigated the "loss" of the twins phenomenon by applying MD simulation on compression of $\mathrm{Mg}$ single crystals. Their result showed that pyramidal $<\mathrm{a}+\mathrm{C}\rangle$ is the main deformation mechanism under c-axis compression at the nano-scale. As they concluded based on their MD simulation results, no twins should occur under the c-axis compression in a perfect magnesium single crystal at the nano-scale, while twinning dominates the defor- 

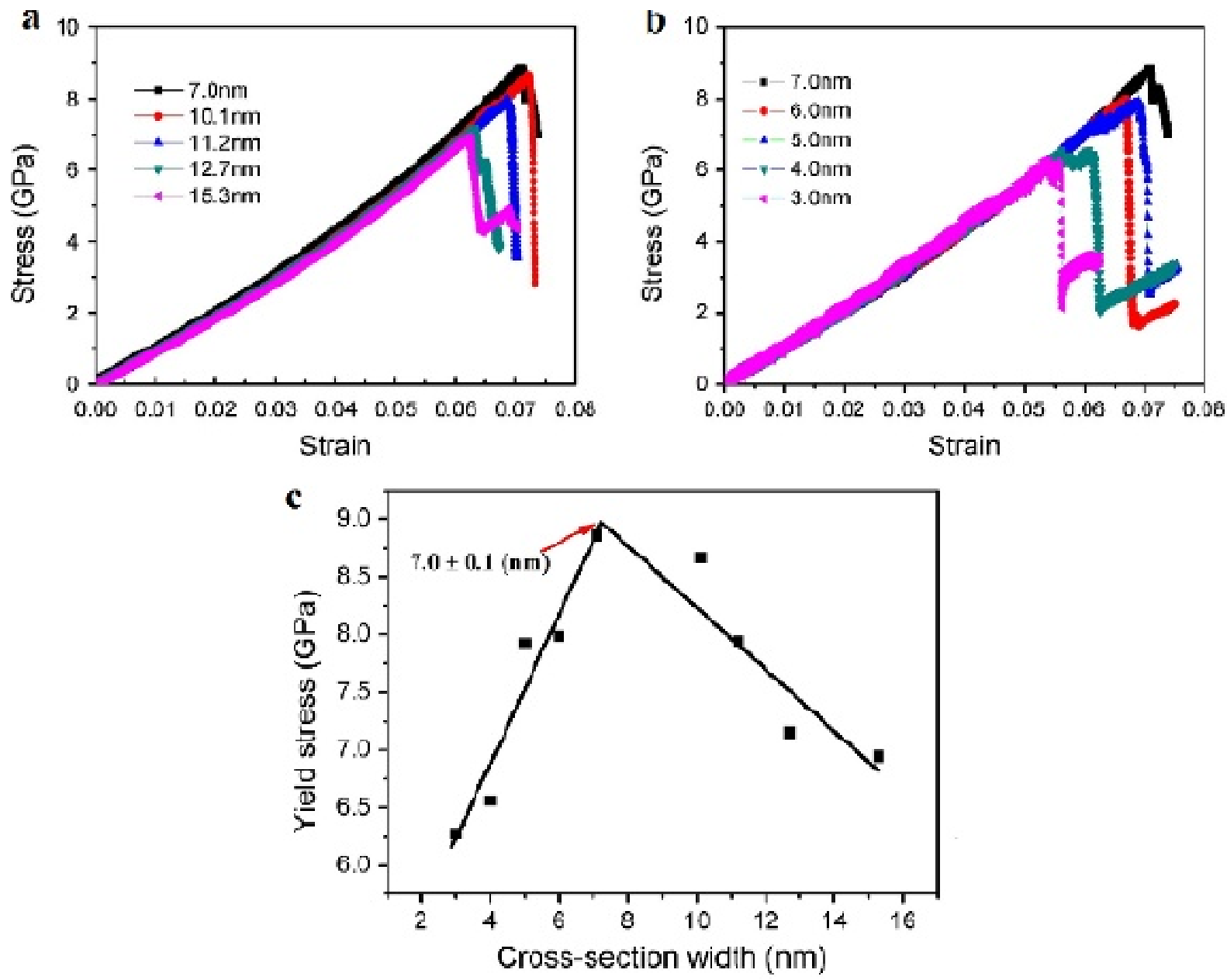

Fig. 36. Compressive stress-strain curves of Ti nanopillars with a) widths greater than $7 \mathrm{~nm}$ and (b) less than $7 \mathrm{~nm}$, (c) the effect of pillar width on the yield strength. Reprinted with permission from Ren JQ, Sun QY, Xiao L, Ding XD, Sun J. 2013. Molecular dynamics simulations of the size effect of titanium singlecrystal nanopillars orientated for double prismatic slips. Philosophical Magazine Letters 93, [262]. ( 2013 Informa UK Lt.

mation under the c-axis tension. This means that the confounded tension-compression asymmetry for magnesium and its alloys is closely related with the difference of deformation mechanisms, respectively, under tension and compression [261].

Ren et al. [262] predicted an inverse "smaller is stronger" effect (Fig. 36) in Ti nanopillars with widths less than $7 \mathrm{~nm}$ that are orientated for double prism slips. This prediction was attributed to the surface effect caused by a thermal vibration of the surface atoms. As they described, since surface becomes the primary dislocation source in the defect-free nanopillars, the plasticity of the pillars is controlled by the edge dislocations, which nucleate on the surface when the pillar width is less than $7 \mathrm{~nm}$. This critical size is dependent to the pillar orientation [172].

Byer and Ramesh [171] investigations on the effects of the initial dislocation density showed that decreasing the initial dislocation density in single crystal magnesium results in a stronger size effect in terms of both increased strength and stochasticity with decreasing pillar size. As shown in a simple schematic in Fig. 37, at relatively larger scales, as the size of the specimen decreases and the number of preexisting sources (or "promoters") of twinning formation reduces, the formation of twinning becomes harder and harder. The effect of size reduction is more significant on the required stress for nucleation of deformation twining than its effect on the required stress for dislocation nucleation and motion [59]. As shown on Fig. 37, there is a size regime where the stress required for deformation twinning is higher than that for nonbasal $<\mathrm{C}+\mathrm{a}>$ dislocations, which would explain the lack of twins seen in the micrometer-scale Mg pillars [60,61]. Simultaneously, as the sample size decreases, the effect of surface area increases and leads to easier 


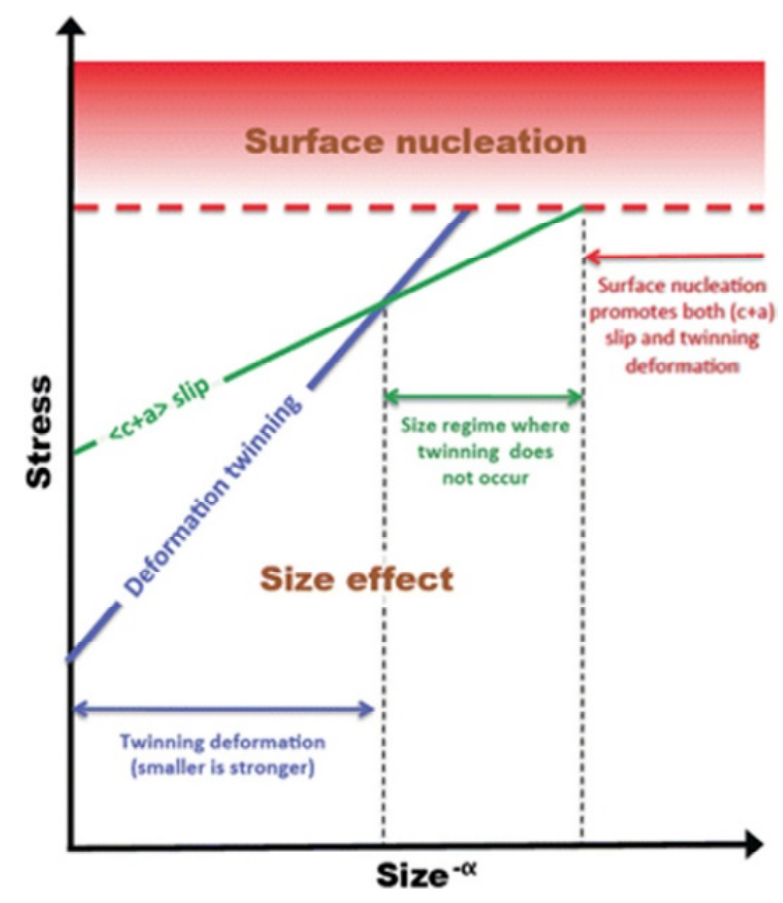

Fig. 37. A schematic diagram presenting the correlation of the size and surface effects on the deformation twinning. á is a positive exponent, $0<\alpha \leq 1$. Reprinted with permission from Yu Q, Mishra RK, Minor AM. 2012. The Effect of Size on the Deformation Twinning Behavior in Hexagonal Close-Packed Ti and Mg. Jom 64, [64]: @ 2012, Springer Nature.

nucleation of defects. So, at the smallest sizes, the easy nucleation of twinning dislocations at the surface can again lead to the appearance of deformation twins, but the relative stress levels for the various mechanisms is different.

Zhang et al. [66] presented mechanism maps that provides regimes of dominant deformation modes that govern the macroscopic yield stress in HCP single crystals as a function of specimen size, source density and macroscopic stress. Fig. 38 shows their presented mechanism map which incorporates all the deformation modes (slip, twin nucleation and twin growth) based on a 95\% deviation of the weakest source distribution as a function of sample size and applied stress. They used the reported experimental to verify their mechanism maps and the expected deformation modes corroborated reasonably well with experimentally observed modes for Mg [171,254,263] and Ti-alloy [59] ) single crystals.

\section{CONCLUSION}

Advent of in situ experiments and improvement of simulation techniques has provided required tools to understand plasticity and deformation mechanisms of small scales. Between the mechanical tests, the nano-compression has been mainly used to understand the behavior of small scale single crystals. The following conclusions are drawn from the current literature on the subject:

- While size effect in small scale is not linked to a specific fabrication technique, the FIB milling introduced defects to the sample could result in lower observed strength than the near theoretical strength supposed to be observed in a perfect crystal. The influence of this damaged surface layer increases with decreasing specimen size and could result in measuring the composite mechanical response of a material and its FIB-modified surface rather than measuring the intrinsic properties of a material.

- Plasticity of small scale fcc metals depends on the dislocation density as well as surface and interior sources of dislocation nucleation. Depending on the initial dislocation density, there is a critical size for the submicron and nano-scale samples, below which the dislocation loss rate will exceed the multiplication rate and thus nucleation of surface dislocations and dislocation starvation hardening will likely dominate the plastic deformation process. Otherwise, multiplication of internal dislocation sources (appears as single arm sources at small scales) and the length of effective source should control the plastic flow. However, in some sizes, both mechanisms occur in a stochastic way.

- While dislocation-mediated deformation is expected to become inactive below a critical size (between 10 to $30 \mathrm{~nm}$ ), some simulation results indicating the effect of strain rate, describing that "liquid-like" deformation is expected to be observed under low strain rate. However, in high-speed deformation, dislocation rebounding at surface and multiple dislocation generation through self-dissociation will result in rapid proliferation of dislocations, and as such may play a role in high-stress and highstrain-rate plastic deformation.

- The size scale of bcc small samples is highly dependent on the deformation temperature as their deformation mainly governs by screw dislocations and the Peierls stress is strongly depends on the temperature. With decreasing critical temperature or pillar diameter, the effect of relative screw dislocation immobility becomes less important (due to the kinetic pile-ups of screw dislocations and kink nucleation at the surface) and consequently the strength of bcc pillars are determined by dislocation starvation model like fcc metals. Therefore, some bcc metals with low $T c$, such as V, display disloca- 


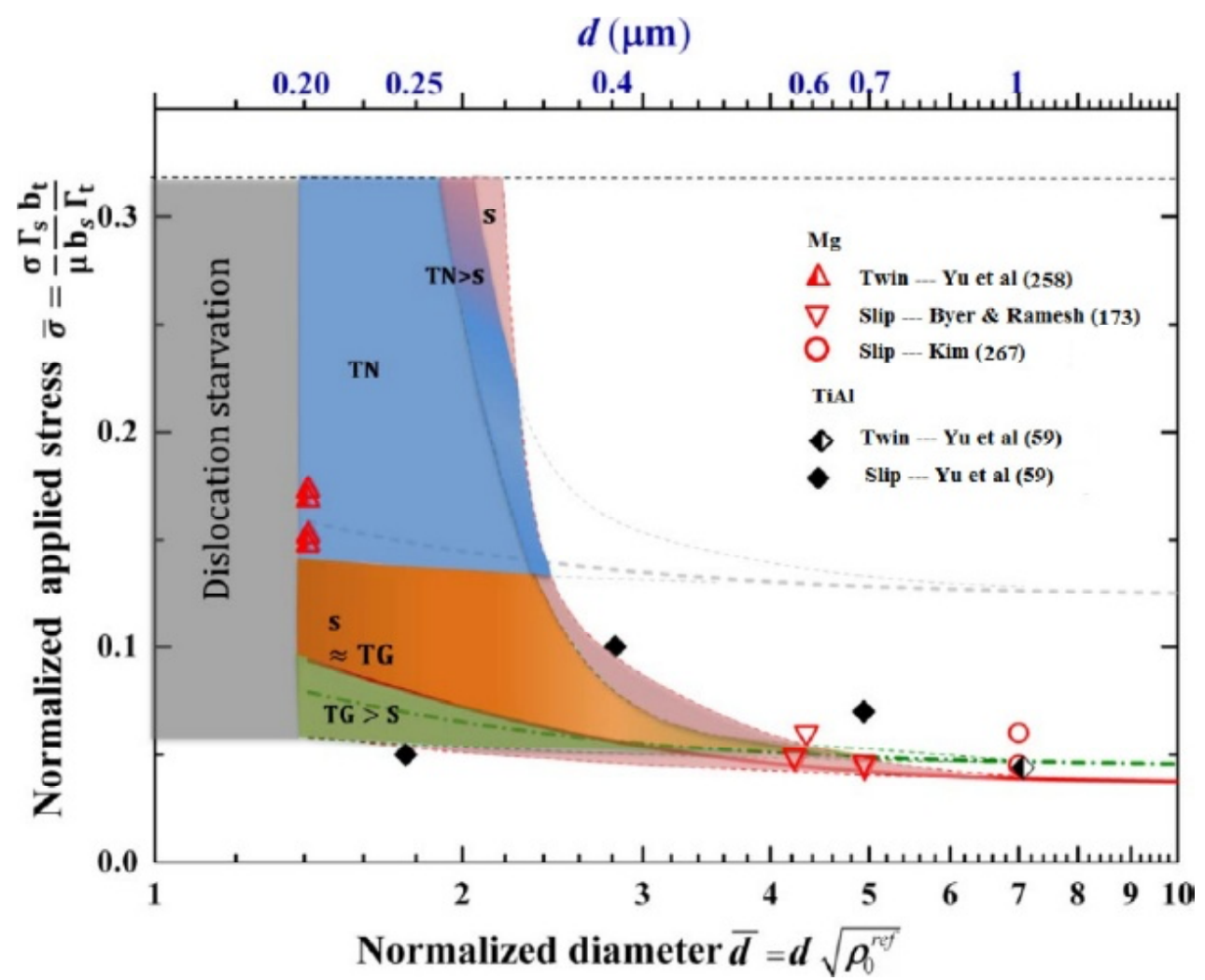

Fig. 38. Mechanism map incorporating all deformation modes of hcp single crystals (slip, twin nucleation and twin growth) based on a 95\% deviation of the weakest source distribution as function of sample diameter $\mathrm{d}$ and applied stress. The symbols in red represent the normalized experimental data of $\mathrm{Mg}$ and the black symbols denote the normalized experimental data of Ti alloy. The open symbol indicates that initial yield is attributed to the slip mode and half-filled symbol indicates that initial yield is attributed to the twinning mode. Reprinted with permission from Zhang J, Ramesh KT, Joshi SP. 2014. Stochastic sizedependent slip-twinning competition in hexagonal close packed single crystals. Modelling and Simulation in Materials Science and Engineering 22, [66]: @ Copyright 2014 IOP Publishing.

tion starvation mechanism, due to their low sp which facilitates dislocation motion and its easier removal from the body of the nanopillar. At temperature lower than Tc, mechanism is quite the opposite of fcc metals and rather than annihilating at the free surfaces, dislocations in bcc metals have been found to pile-up in the vicinity of dislocation sources and interact with the sample surface and form intricate networks as a result of mechanical deformation even in the smallest pillar size of $\sim 200 \mathrm{~nm}$ diameter.

- The required stress for deformation twinning in hcp singe crystals increases with decreasing sample size, until the sample size is reduced to a critical size (near one micrometer), depending to the composition as well as kinetic factors such as temperature and strain rate, below which the deformation twinning is entirely replaced by dislocation plasticity. As the sample size decreases to nanometerscale, the effect of surface area increases and results in easy nucleation of twinning dislocations at the surface and deformation twins appear again in nano-sized samples. However, orientation plays an important role in small scale plasticity of hcp metals as basal slip oriented micro crystals deform only by slip while twin oriented crystals deform by both slip and twinning modes. It is expected that the effect of orientation becomes less important in nanosized samples.

\section{REFERENCES}

[1] Y. L. Tang, Y. T. He, G. Z. Liu, J. X. Fu, H. Sun, K. Zhang and Y. H. Wu // Adv Mater Res-Switz 211-212 (2011) 742.

[2] R. C. Cammarata // Prog Surf Sci 46 (1994) 1.

[3] C. L. Kelchner, S. J. Plimpton and J. C. Hamilton // Phys Rev B 58 (1998) 11085.

[4] L. Pizzagalli, S. Brochard, J. Godet, C. Gérard, In: Mechanical Properties of Nanostructures: Encyclopedia of Nanotechnology, ed. by B. Bhushan (Springer, Dordrecht, 2015), p. 1945. 
[5] W. D. Nix and S. W. Lee // Philos Mag 91 (2011) 1084.

[6] J. R.Greer and W. D. Nix // Phys Rev B 73 (2006) 245410.

[7] H. Van Swygenhoven, P. M. Derlet and A. G. Froseth // Nat Mater 3 (2004) 399.

[8] M. W. Chen, E. Ma, K. J. Hemker, H. W. Sheng, Y. M. Wang and X. M. Cheng // Science 300 (2003) 1275.

[9] L. Lu, Y. F. Shen, X. H. Chen, L. H. Qian and K. Lu // Science 304 (2004) 422.

[10] L. Lu, R. Schwaiger, Z. W. Shan, M. Dao, K. Lu and S. Suresh // Acta Mater 53 (2005) 2169.

[11] E. Ma, Y. M. Wang, Q. H. Lu, M. L. Sui, L. Lu and K. Lu // Appl Phys Lett 85 (2004) 4932.

[12] W. S. Zhao, N. R. Tao, J. Y. Guo, Q.H. Lu and K. Lu // Scripta Mater 53 (2005) 745.

[13] D. Xu, W. L. Kwan, K. Chen, X. Zhang, V. Ozolins and K.N. Tu // Appl Phys Lett 91 (2007) 254105.

[14] T. Zhu, J. Li, A. Samanta, H. G. Kim and S. Suresh // P Natl Acad Sci USA 104 (2007) 3031.

[15] L. Lu, X. Chen, X. Huang and K. Lu // Science 323 (2009) 607.

[16] Y. Zhang, Y. S. Li, N. R. Tao and K. Lu // Appl Phys Lett 91 (2007) 21901.

[17] K. Lu, L. Lu and S. Suresh // Science 324 (2009) 349.

[18] T. H. Fang, W. L. Li, N. R. Tao and K. Lu // Science 331 (2011) 1587.

[19] H. T. Wang, N. R. Tao and K. Lu // Acta Mater60 (2012) 4027.

[20] Lu, K., Yan, F. K., Wang, H. T., Tao, N. R. Scripta Mater 2012, 66, (11), 878-883.

[21] S. J. Zhu, J. H. Zhang, S. J. Tang, C. Y. Qiao, L. Wang, H. Y. Wang, X. Liu, B. Li, Y. F. Li, W. L. Yu, X. F. Wang, H. C. Sun and B. Yang // Adv Funct Mater 22 (2012) 4732.

[22] Y. J. Tian, B. Xu, D. L. Yu, Y. M. Ma, Y. B. Wang, Y. B. Jiang, W. T. Hu, C. C. Tang, Y. F. Gao, K. Luo, Z. S. Zhao, L. M. Wang, B. Wen, J. L. He and Z. Y. Liu // Nature 493 (2013) 385.

[23] R. G. Li, H. J. Kang, Z. N. Chen, G. H. Fan, C. L. Zou, W. Wang, S. J. Zhang, Y. P. Lu, J. C. Jie, Z. Q. Cao, T. J. Li and T. M. Wang // Sci Rep-Uk 6 (2016) 20799.

[24] K. Lu // Nat Rev Mater 1 (2016) 16019.

[25] S. W. Lee, S. M. Han and W. D. Nix // Acta Mater 57 (2009) 4404.
[26] J. A. Brown and N. M. Ghoniem // Acta Mater $58(2010) 886$.

[27] J. P. Hirth // Cc/Phys Chem Earth 25 (1981) 18.

[28] Z. W. Shan, R. K. Mishra, S. A. S. Asif, O. L. Warren and A. M. Minor // Nat Mater 7 (2008) 115.

[29] A. T. Jennings, J. Li and J. R. Greer // Acta Mater 59 (2011) 5627.

[30] J. R. Greer, C. R. Weinberger and W. Cai // Mat Sci Eng a-Struct 493 (2008) 21.

[31] C. A. Volkert and E. T. Lilleodden // Philos Mag 86 (2006) 5567.

[32] W. D. Nix, J. R. Greer, G. Feng and E. T. Lilleodden // Thin Solid Films 515 (2007) 3152.

[33] D. Maharaj and B. Bhushan // Beilstein J Nanotechnol5 (2014) 822.

[34] D. Mordehai, M. Kazakevich, D. J. Srolovitz and E. Rabkin // Acta Mater 59 (2011) 2309.

[35] M. D. Uchic, D. M. Dimiduk, J. N. Florando and W. D. Nix // Science 305 (2004) 986.

[36] J. R. Greer, W. C. Oliver and W. D. Nix // Acta Mater 53 (2005) 1821.

[37] E. Rabkin, H. S. Nam and D. J. Srolovitz // Acta Mater 55 (2007) 2085.

[38] H. Tang, K. W. Schwarz and H. D. Espinosa // Acta Mater 55 (2007) 1607.

[39] C. R. Weinberger and W. Cai // P Natl Acad Sci USA 105 (2008) 14304.

[40] J. Y. Kim and J. R. Greer // Appl Phys Lett 93 (2008) 101916.

[41] S. Brinckmann, J. Y. Kim and J. R. Greer // Phys Rev Lett 10 (2008) 155502.

[42] K. A. Afanasyev and F. Sansoz // Nano Lett 7 (2007) 2056.

[43] J. R. Greer and J. T. M. De Hosson // Prog Mater Sci56 (2011) 654.

[44] E. Rabkin and D. J. Srolovitz // Nano Lett 7 (2007) 101.

[45] S. R. Jian // J Alloy Compd 644 (2015) 54.

[46] O. Franke, J. Alcala, R. Dalmau, Z. C. Duan, J. Biener, M. M. Biener and A. M. Hodge // Philos Mag 95 (2015) 1866.

[47] S. Pathak and S. R. Kalidindi // Mat Sci Eng $R 91$ (2015) 1.

[48] J. Y. Kim, D. C. Jong and J. R. Greer // Acta Mater 58 (2010) 2355.

[48] J. Y. Kim, D. C. Jang and J. R. Greer // Scripta Mater61 (2009) 300.

[50] D. Mordehai, S. W. Lee, B. Backes, D. J. Srolovitz, W. D. Nix and E. Rabkin // Acta Mater 59 (2011) 5202. 
[51] D. M. Dimiduk, M. D. Uchic and T. A. Parthasarathy // Acta Mater 53 (2005) 4065.

[52] A. T. Jennings, M. J. Burek and J. R. Greer // Phys Rev Lett 104 (2010) 135503.

[53] S. Xu, Y. F. Guo and A. H. W. Ngan // Int J Plasticity 43 (2013) 116.

[54] J. M. Wheeler, C. Kirchlechner, J.-S. Micha, J. Michler and D. Kiener // Philos Mag 96 (2016) 1.

[55] A. S. Schneider, D. Kaufmann, B. G. Clark, C. P. Frick, P. A. Gruber, R. Monig, O. Kraft and E. Arzt // Phys Rev Lett 103 (2009) 105501.

[56] S. M. Han, T. Bozorg-Grayeli, J. R. Groyes and W. D. Nix // Scripta Mater 63 (2010) 1153.

[57] S. M. Han, G. Feng, J. Y. Jung, H. J. Jung, J. R. Groves, W. D. Nix and Y. Cui / App/ Phys Lett 102 (2013) 041910.

[58] O. T. Abad, J. M. Wheeler, J. Michler, A. S. Schneider and E. Arzt // Acta Mater 103 (2016) 483.

[59] Q. Yu, Z. W. Shan, J. Li, X. X. Huang, L. Xiao, J. Sun and E. Ma // Nature 463 (2010) 335.

[60] Q. Y. Sun, Q. Guo, X. Yao, L. Xiao, J. R. Greer and J. Sun // Scripta Mater 65 (2011) 473.

[61] E. Lilleodden // Scripta Mater 62 (2010) 532.

[62] C. M. Byer, B. Li, B. Y. Cao and K. T. Ramesh // Scripta Mater 62 (2010) 536.

[63] J. Ye, R. K. Mishra, A. K. Sachdev and A. M. Minor // Scripta Mater 64 (2011) 292.

[64] Q. Yu, R. K. Mishra and A. M. Minor // JomUs 64 (2012) 1235.

[65] S. Karewar, N. Gupta, S. Groh, E. Martinez, A. Caro and S. G. Srinivasan // Comp Mater Sci 126 (2017) 252.

[66] J. Zhang, K. T. Ramesh and S. P. Joshi // Model Simul Mater Sc 22 (2014) 075003.

[67] M. J. Burek, A. S. Budiman, Z. Jahed, N. Tamura, M. Kunz, S. M. Jin, S. M. J. Han, G. Lee, C. Zamecnik and T.Y. Tsui // Mat Sci Eng a-Struct 528 (2011) 5822.

[68] N. Zhang and M. Asle Zaeem // Acta Mater 120 (2016) 337.

[69] G. Lee, J. Y. Kim, A. S. Budiman, N. Tamura, M. Kunz, K. Chen, M.J. Burek, J.R. Greer and T. Y. Tsui // Acta Mater 58 (2010) 1361.

[70] G. Lee, J. Y. Kim, M. J. Burek, J. R. Greer and T. Y. Tsui // Mat Sci Eng a-Struct $\mathbf{5 2 8}$ (2011) 6112.
[71] A. S. Budiman, G. Lee, M. J. Burek, D. C. Jang, S. M. J. Han, N. Tamura, M. Kunz, J. R. Greer and T. Y. Tsui // Mat Sci Eng a-Struct 538 (2012) 89.

[72] M. J. Burek, S. M. Jin, M. C. Leung, Z. Jahed, J. Wu, A. S. Budiman, N. Tamura, M. Kunz and T. Y. Tsui // Acta Mater 59 (2011) 4709.

[73] S. M. Jin, M. J. Burek, N. D. Evans, Z. Jahed and T. Y. Tsui // Scripta Mater 66 (2012) 619.

[74] M. D. Uchic, P. A. Shade and D. M. Dimiduk // Annu Rev Mater Res 39 (2009) 361.

[75] A. C. Fischer-Cripps, Nanoindentation (Springer, New York, 2002).

[76] A. T. Jennings and J. R. Greer // Philos Mag 91 (2011) 1108.

[77] S. Barakat, P. Lee-Sullivan, S. A. Vitale and T. Y. Tsui // J Mater Res 26 (2011) 2524.

[78] L. Yang, S. G. Long, Z. S. Ma and Z. H. Wang // T Nonferr Metal Soc 24 (2014) 3265.

[79] O. Alshehri, Mechanical Behaviour of Nanocrystalline Rhodium Nanopillars under Compression (University of Waterloo, Waterloo, Ontario, Canada, 2012).

[80] W. C. Oliver and J. B. Pethica, Method for continuous determination of the elastic stiffness of contact between two bodies, US Patent No. 4,848,141; 1989.

[81] J. B. Pethica, R. Hutchings and W. C. Oliver // Philos Mag A 48 (1983) 593.

[82] W. C. Oliver and G. M.Pharr // Mrs Bull 35 (2010) 897.

[83] H. Nili, K. Kalantar-zadeh, M. Bhaskaran and S. Sriram // Prog Mater Sci 58 (2013) 1.

[84] C. Rudolf, B. Boesl and A. Agarwal // Jom-Us 68 (2016) 136.

[85] R. Ghisleni, K. Rzepiejewska-Malyska, L. Philippe, P. Schwaller and J. Michler // Microsc Res Techniq 72 (2009) 242.

[86] R. Rabe, J. M. Breguet, P. Schwaller, S. Stauss, F. J. Haug, J. Patscheider and J. Michler // J. Thin Solid Films 469 (2004) 206.

[87] X. W. Gu, M. Jafary-Zadeh, D. Z. Chen, Z. X. Wu, Y. W. Zhang, D. J. Srolovitz and J. R. Greer // Nano Lett 14 (2014) 5858.

[88] M. L. B. Palacio and B. Bhushan // Mater Charact 78 (2013) 1.

[89] Q. N. Han, W. H. Qiu, Y. B. Shang and H. J. Shi // Tribol Int 101 (2016) 33.

[90] B. Moser, J. Kuebler, H. Meinhard, W. Muster and J. Michler // Adv Eng Mater 7 (2005) 388. 
[91] Q. P. Cao, J. W. Liu, K. J.Yang, F. Xu, Z. Q. Yao, A. Minkow, H. J. Fecht, J. Ivanisenko, L. Y. Chen, X. D. Wang, S. X. Qu and J. Z. Jiang // Acta Mater 58 (2010) 1276.

[92] D. Z. Chen, D. Jang, K. M. Guan, Q. An, W. A. Goddard and J. R. Greer // Nano Lett 13 (2013) 4462.

[93] H. E. Weekes, V. A. Vorontsov, I. P. Dolbnya, J. D. Plummer, F. Giuliani, T. B. Britton and

D. Dye // Acta Mater 92 (2015) 81.

[94] J. Y. Kim and J. R. Greer // Acta Mater 57 (2009) 5245.

[95] S. W. Lee, Y. T. Cheng, I. Ryu and J. R. Greer // Sci China Technol Sc 57 (2014) 652.

[96] S. W Lee, L. Meza and J. R. Greer // App/ Phys Lett 103 (2013) 101906.

[97] Q. Yu, M. Legros and A. M. Minor // Mrs Bull 40 (2015) 62.

[98] M. Legros // Cr Phys 15 (2014) 224.

[99] E. T. Selig and R. S. Ladd, Evaluation of relative density and its role in geotechnical projects involving cohesionless soils (American Society for Testing and Materials, Philadelphia, 1973).

[100] J. Wang and S. X. Mao // Extreme Mechanics Letters 8 (2016) 127.

[101] S. H. Oh, M. Legros, D. Kiener and G. Dehm // Nat Mater 8 (2009) 95.

[102] J. Wang, Z. Zeng, C. R. Weinberger, Z. Zhang, T. Zhu and S. X. Mao // Nat Mater 14 (2015) 594.

[103] D. Kiener, P. Hosemann, S. A. Maloy and A. M. Minor // Nat Mater 10 (2011) 608.

[104] H. Zheng, A. J. Cao, C. R. Weinberger, J. Y. Huang, K. Du, J. B. Wang, Y. Y. Ma, Y. N. Xia and S. X. Mao // Nat Commun 1 (2010) 144.

[105] Z. W. Shan // Jom-Us 64 (2012) 1229.

[106] K. Zheng, C. C. Wang, Y. Q. Cheng, Y. H. Yue, X. D. Han, Z. Zhang, Z. W. Shan, S. X. Mao, M. M. Ye, Y. D. Yin and E. Ma // Nat Commun 1 (2010) 24.

[107] R. Kositski, O. Kovalenko, S. W. Lee, J. R. Greer, E. Rabkin and D. Mordehai // Sci Rep-Uk 6 (2016) 25966.

[108] D. Liu, Mechanical characterization of isolated nanostructures (Rensselaer Polytechnic Institute, Troy, New York, 2004).

[109] S. Varghese, New method for estimating local strains using nanoindentation and atomic force microscopy (Oklahoma State University, 2006).
[110] X. Wei, Mechanical properties of nanoscale and atomic scale materials: Nanocrystalline copper and graphene (Columbia University, 2009).

[111] W. H. Roos, R. Bruinsma and G. J. L. Wuite // Nature Physics 6 (2010) 733.

[112] K. Daeinabi and M. H. Korayem // Journal of Nanoparticle Research 13 (2010) 1075.

[113] B. Gan, H. Murakami, R. Maass, L. Meza, J. R. Greer, T. Ohmura and S. Tin // Superalloys 2012 (2012) 83.

[114] E. L. Wood, T. Avant, G. S. Kim, S. K. Lee, Z. Burchman, J. M. Hughes and F. Sansoz // Acta Mater 66 (2014) 32.

[115] L. T. Sun, A. V. Krasheninnikov, T. Ahlgren, K. Nordlund and F. Banhart // Phys Rev Lett 101 (2008) 156101.

[116] K. J. Hemker and W. D. Nix // Nat Mater 7 (2008) 97.

[117] P. B. Fischer and S. Y. Chou // Microelectron Eng 21 (1993) 311.

[118] W. Chen and H. Ahmed // Appl Phys Lett 63 (1993) 1116.

[119] C. Vieu, F. Carcenac, A. Pepin, Y. Chen, M. Mejias, A. Lebib, L. Manin-Ferlazzo, L. Couraud and H. Launois // App/ Surf Sci 164 (2000) 111.

[120] J. L. Duvail, S. Dubois, L. Piraux, A. Vaures, A. Fert, D. Adam, M. Champagne, F. Rousseaux and D. Decanini // J Appl Phys 84 (1998) 6359.

[121] J. B. Cui and U. Gibson // J. Zinc Oxide and Related Materials 957 (2007) 201.

[122] H. Kim, S. C. Shin, J. Han, J. Han, S. Kang and T Lee // Magn 45 (2009) 2304.

[123] J. S. Wi, H. S. Lee, K. Lim, S. W. Nam, H. M. Kim, S. Y. Park, J. J. Lee, C. D. Hong, S. Jin and K. B. Kim // Small 4 (2008) 2118.

[124] R. M. Ostrom, D. M. Tanenbaum and A. Gallagher // App/ Phys Lett 61 (1992) 925.

[125] G. M. S Russell // Nanotechnology 1 (1990) 67.

[126] T. Tada and T. Kanayama // Jpn J App/ Phys 34 (1995) 6947.

[127] X. H. Jiang, G. Y. Wu, J. F. Zhou, S. J. Wang, A. A. Tseng and Z. L. Du // Nanoscale Res Lett 6 (2011) 457.

[128] T. Tada, T. Kanayama, K. Koga, P. Weibel, S. J. Carroll, K. Seeger and R. E. Palmer // J Phys D Appl Phys 31 (1998) L21. 
[129] T. Tada and T. Kanayama // J Vac Sci Technol B 16 (1998) 3934.

[130] P. A. Lewis, H. Ahmed and T. Sato // J Vac Sci Technol B 16 (1998) 2938.

[131] C. L. Cheung, R. J. Nikolic, C. E. Reinhardt and T. F. Wang // Nanotechnology 17 (2006) 1339.

[132] J. W. Yang, J. I. Sim, H. M. An and T. G. Kim // J Korean Phys Soc 58 (2011) 994.

[133] W. Li, W. M. Zhao and P. Sun // Physica E 41 (2009) 1600.

[134] W. Li, L. Xu, W. M. Zhao, P. Sun, X. F. Huang and K.Chen // J. App/ Surf Sci 253 (2007) 9035.

[135] A. Sinitskii, S. Neumeier, J. Nelles, M. Fischler and U. Simon // Nanotechnology 18 (2007) 305307.

[136] R. Sanatinia, M. Swillo and S. Anand // Nano Lett 12 (2012) 820.

[137] Z. Szabo, J. Volk, E. Fulop, A. Deak and I. Barsony // Photonic Nanostruct 11 (2013) 1.

[138] A. Cowley, J. A.Steele, D. Byrne, R. K. Vijayaraghavan and P. McNally // J. Rsc Adv 6 (2016) 30468.

[139] A. J. Pedraza, J. D. Fowlkes and D. H. Lowndes // Appl Phys Lett 74 (1999) 2322.

[140] Y. Belaroussi, T. Kerdja, C. Yaddadene, A. Djemaa, A. Keffous and N. Gabouze // J Cryst Growth 337 (2011) 20.

[141] A. Djemaa, N. Gabouze, C. Yaddadene, Y. Belaroussi, T. Kerdja, M. Kechouane, H. Menari and A. Y. Khereddine // Surf Interface Anal 42 (2010) 1295.

[142] O. V. Kuzmin, Y. T. Pei and J. T. M. De Hosson // Microsc Microanal 20 (2014) 1581.

[143] C. A. Volkert and A. M. Minor // Mrs Bull 32 (2007) 389.

[144] M. D. Uchic and D. A. Dimiduk // Mat Sci Eng a-Struct 400 (2005) 268.

[145] C. P. Frick, B. G. Clark, S. Orso, A. S. Schneider and E. Arzt // Materials Science and Engineering: A 489 (2008) 319.

[146] H. Bei and S. Shim // App/ Phys Lett 91 (2007) 111915.

[147] F. Ostlund, K. Rzepiejewska-Malyska, K. Leifer, L. M. Hale, Y. Y. Tang, R. Ballarini, W. W. Gerberich and J. Michler // Adv Funct Mater 19 (2009) 2439.

[148] D. Kiener, C. Motzand G. Dehm // Mat Sci Eng a-Struct 505 (2009) 79.
[149] J. Jeong, S. Lee, Y. Kim, S. M. Han, D. Kiener, Y. B. Kang and S. H. Oh // Scripta Mater 86 (2014) 44.

[150] J. Mayer, L. A. Giannuzzi, T. Kamino and J. Michael // Mrs Bull 32 (2007) 400.

[151] J. P. McCaffrey, M. W. Phaneuf and L. D. Madsen // Ultramicroscopy 81 (2001) 97.

[152] D. Kiener, C. Motz, M. Rester, M. Jenko and G. Dehm // Mat Sci Eng a-Struct 459 (2007) 262.

[153] D. M. Norfleet, D. M. Dimiduk, S. J. Polasik, M. D. Uchic and M. J. Mills // Acta Mater56 (2008) 2988.

[154] S. Korte, J. S. Barnard, R. J. Steam and W. J.Clegg // Int J Plasticity 27 (2011) 1853.

[155] R. Maass, D. Grolimund, S. Van Petegem, M. Willimann, M. Jensen, $H$. Van Swygenhoven, T. Lehnert, M. A. M. Gijs, C. A. Volkert, E. T. Lilleodden and R. Schwaiger // Appl Phys Lett 89 (2006) 151905.

[156] K. Thompson, D. Lawrence, D. J. Larson, J. D. Olson, T. F. Kelly and B. Gorman // Ultramicroscopy 107 (2007) 131.

[157] S. W. Lee, D. Mordehai, E. Rabkin and W. D. Nix // J Mater Res 26 (2011) 1653.

[158] D. Kiener, C. Motz, T. Schoberl, M. Jenkoand G. Dehm // Adv Eng Mater 8 (2006) 1119.

[159] C. P. Frick, S. Orso and E. Arzt // Acta Mater55 (2007) 3845.

[160] R. Dou and B. Derby // Scripta Mater 61 (2009) 524.

[161] R. Maass, C. A. Volkert and P. M. Derlet // Scripta Mater 102 (2015) 27.

[162] R. Gu and A. H. W. Ngan // Scripta Mater 68 (2013) 861.

[163] A. Kunz, S. Pathak and J. R. Greer // Acta Mater59 (2011) 4416.

[164] A. S. Schneider, B. G. Clark, C. P. Frick, P. A. Gruber and E. Arzt // Mat Sci Eng a-Struct 508 (2009) 241.

[165] Q. Y.Sun, Q. Guo, X. Yao, L. Xiao, J. R. Greer and J. Sun // Scripta Mater 65 (2011) 935.

[166] A. S. Schneider, C. P. Frick, B. G. Clark, P. A. Gruber and E. Arzt // Mat Sci Eng a-Struct 528 (2011) 1540.

[167] A. S. Schneider, C. P. Frick, E. Arzt, W. J.Clegg and S. Korte // Phil Mag Lett 93 (2013) 331. 
[168] D. Kaufmann, A. S. Schneider, R. Monig, C. A. Volkert and O. Kraft // Int J Plasticity 49 (2013) 145.

[169] D. Kaufmann, R. Monig, C. A. Volkert and O. Kraft // Int J Plasticity 27 (2011) 470.

[170] Z. H. Aitken, H. D. Fan, J. A. El-Awady and J. R. Greer // J Mech Phys Solids 76 (2015) 208.

[171] C. M. Byer and K. T. Ramesh // Acta Mater $61(2013) 3808$.

[172] J. Q. Ren, Q. Y. Sun, L. Xiao, X. D. Ding and J. Sun // Mat Sci Eng a-Struct 615 (2014) 22.

[173] S. Shim, H. Bei, M. K. Miller, G. M. Pharr and E. P. George // Acta Mater 57 (2009) 503.

[174] S. Lee, J. Jeong, Y. Kim, S. M. Han, D. Kiener and S. H. Oh // Acta Mater 110 (2016) 283.

[175] M. J. Burekand J. R. Greer // Nano Lett 10 (2010) 69.

[176] J. R. Greer, Fabrication of vertically aligned metallic nanopillars, US20100291385 A1, Nov 18, 2010.

[177] O. Alshehri, M. Yavuz and T. Tsui // Acta Mater61 (2013) 40.

[178] M. Dietiker, S. Buzzi, G. Pigozzi, J. F. Löffler and R. Spolenak // Acta Mater 59 (2011) 2180.

[179] D. Kiener, W. Grosinger, G. Dehm and R. Pippan // Acta Mater 56 (2008) 580.

[180] S.-W. Lee and W. D. Nix // Philos Mag 92 (2012) 1238.

[181] A. T. Jennings, Deformation mechanisms in nanoscale single crystalline electroplated copper pillars (California Institute of Technology, Pasadena, California, 2012).

[182] K. Shreiber and D. Mordehai // Model Simul Mater Sc 23 (2015) 085004.

[183] J. R. Greer and W. D. Nix // Appl Phys a-Mater 90 (2008) 203.

[184] Julia R. Greer // Rev Adv Mater Sci 13 (2006) 59.

[185] A. Jerusalem, A. Fernandez, A. Kunz and J. R. Greer // Scripta Mater 66 (2012) 93.

[186] T. Zhu, J. Li, A. Samanta, A. Leach and K. Gall // Phys Rev Lett 10 (2008) 025502.

[187] P. A. Gruber, C. Solenthaler, E. Arzt and R. Spolenak // Acta Mater 56 (2008) 1876.

[188] S. H.Oh, M. Legros, D. Kiener, P. Gruber and G. Dehm // Acta Mater 55 (2007) 5558.

[189] J. D. Kiely and J. E. Houston // Phys Rev B 57 (1998) 12588.
[190] J. D. Kiely, R. Q. Hwang and J. E. Houston // Phys Rev Lett 81 (1998) 4424.

[191] A.Gouldstone, K. J. Van Vliet and S. Suresh // Nature 411 (2001) 656.

[192] J. A. Zimmerman, C. L. Kelchner, P. A. Klein, J. C. Hamilton and S. M. Foiles // Phys Rev Lett 87 (2001) art. no.-165507.

[193] H. B. Lu and Y. S. Ni // Thin Solid Films 520 (2012) 4934.

[194] D. B. Shan, L. Yuan and B. Guo // Mat Sci Eng a-Struct 412 (2005) 264.

[195] M. R. Shankar // Appl Phys Lett 90 (2007) 171924.

[196] C. Li and G. Xu // Philos Mag 86 (2006) 2957.

[197] V. Navarro, O. R. de la Fuente, A. Mascaraque and J. M. Rojo // Phys Rev Lett 100 (2008) 105504.

[198] Z.-J. Wang, Q.-J. Li, Z.-W. Shan, J. Li, J. Sun and E. Ma // Appl Phys Lett 100 (2012) 071906.

[199] C. Z.Zhou, S. B. Biner and R. LeSar // Acta Mater58 (2010) 1565.

[200] C. Z. Zhou, I. J. Beyerlein and R. LeSar // Acta Mater 59 (2011) 7673.

[201] J. J. Gilman // Science 306 (2004) 1134.

[202] I. Ryu, W. Cai, , W. D. Nix and H. Gao // Acta Mater 95 (2015) 176.

[203] T. A. Parthasarathy, S. I. Rao, D. M. Dimiduk, M. D. Uchic and D. R. Trinkle // Scripta Mater 56 (2007) 313.

[204] F. Mompiou, M. Legros, A. Sedlmayr, D. S. Gianola, D. Caillard and O. Kraft // Acta Mater60 (2012) 977.

[205] J. Senger, D. Weygand, P. Gumbsch and O. Kraft // Scripta Mater 58 (2008) 587.

[206] Y. N. Cui, P. Lin, Z. L. Liu and Z. Zhuang // International Journal of Plasticity 55 (2014) 279.

[207] S. W. Lee and W. D. Nix // Mat Sci Eng a-Struct 527 (2010) 1903.

[208] H. D. Fan, Z. H. Li and M. S. Huang // Scripta Mater 67 (2012) 225.

[209] Y. N. Cui, Z. L. Liu and Z. Zhuang // Int J Plasticity 69 (2015) 54.

[210] Y. N. Cui, Z. L. Liu and Z. Zhuang // J Mech Phys Solids 76 (2015) 127.

[211] H. J. Chang, J. Segurado, J. M. MolinaAldareguia, R. Soler and J. LLorca // Model Simul Mater Sc 24 (2016) 035009.

[212] Y. N. Cui, Z. L. Liu, Z. J. Wang and Z. Zhuang // J Mech Phys Solids 89 (2016) 1. 
[213] P. Lin, Z. L. Liu, Y. N. Cui and Z. Zhuang // Int J Solids Struct 69-70 (2015) 267.

[214] S. W. Lee, A. T. Jennings and J. R. Greer // Acta Mater 61 (2013) 1872.

[215] B. Pan, Y. Shibutani, X. Zhang and F. Shang // J Appl Phys 118 (2015) 014305.

[216] C. R. Weinberger and W. Cai // Scripta Mater64 (2011) 529.

[217] E. Tarleton, D. S. Balint, J. Gong and A. J. Wilkinson // Acta Mater 88 (2015) 271.

[218] X. W. Gu, C. N. Loynachan, Z. X. Wu, Y. W. Zhang, D. J. Srolovitz and J. R. Greer // Nano Lett 12 (2012) 6385.

[219] S. I. Rao, D. M. Dimiduk, T. A. Parthasarathy, M. D. Uchic, M. Tang and C. Woodward // Acta Mater 56 (2008) 3245.

[220] J. K. Diao, K. Gall, M. L. Dunn and J. A. Zimmerman // Acta Mater 54 (2006) 643.

[221] J. Q. Hu, Z. L. Liu, Y. N. Cui, Z. J. Wang, Z. W. Shan and Z. Zhuang // J Appl Mech-T Asme 81 (2014) 091007.

[222] Y.Cui, The Investigation of Plastic Behavior by Discrete Dislocation Dynamics for Single Crystal Pillar at Submicron Scale (Springer, Singapore, 2017).

[223] D. Kiener and A. M. Minor // Acta Mater 59 (2011) 1328.

[224] K. Sieradzki, A. Rinaldi, C. Friesen and P. Peralta // Acta Mater 54 (2006) 4533.

[225] N. Agrait, G. Rubio and S. Vieira // Phys Rev Lett 74 (1995) 3995.

[226] K. Gall, J. K. Diao and M. L. Dunn // Nano Lett 4 (2004) 2431.

[227] P. Z.Coura, S. B. Legoas, A. S. Moreira, F.Sato, V. Rodrigues, S. O. Dantas, D. Ugarte and D. S. Galvao // Nano Lett 4 (2004) 1187.

[228] W. Liang and M. Zhou // P I Mech Eng C-J Mec 218 (2004) 599.

[229] M. R. Sorensen, M. Brandbyge and K. W. Jacobsen // Phys Rev B 57 (1998) 3283.

[230] H. S. Park, K. Gall and J. A. Zimmerman // J Mech Phys Solids 54 (2006) 1862.

[231] J. Sun, L. B. He, Y. C. Lo, T. Xu, H. C. Bi, L. T. Sun, Z. Zhang, S. X. Mao and J. Li // Nat Mater 13 (2014) 1007.

[232] C. R. Henry // Nat Mater 13 (2014) 999.

[233] B. Chen, K. Lutker, S. V. Raju, J. Y. Yan, W. Kanitpanyacharoen, J. L. Lei, S. Z. Yang, H. R. Wenk, H. K. Mao and Q. Williams // Science 338 (2012) 1448.

[234] Q. J. Li, J. Li, Z. W. Shan and E. Ma // Acta Mater 119 (2016) 229.
[235] X. Yan and P. Sharma // Nano Lett 16 (2016) 3487.

[236] A. S. Schneider, D. Kiener, C. M. Yakacki, H. J. Maier, P. A. Gruber, N. Tamura, M. Kunz, A. M. Minor, C. P. Frick // Materials Science and Engineering: A 559 (2013) 147.

[237] R. J. Milne, A. J. Lockwood and B. J. Inkson // J Phys D Appl Phys 44 (2011) 485301.

[238] R. Huang, Q. J. Li, Z. J. Wang, L. Huang, J. Li, E. Ma and Z. W. Shan // Mater Res Lett 3 (2015) 121.

[239] L. Huang, Q. J. Li, Z. W. Shan, J. Li, J. Sun and E. Ma // Nat Commun 21 (2011) 2.

[240] J. Y. Kim, D. C. Jang and J. R. Greer // Int J Plasticity 48 (2012) 46.

[241] A. S. Schneider, B. G. Clark, C. P. Frick, P. A. Gruber and E. Arzt // Phil Mag Lett 90 (2010) 841.

[242] M. Zaiser, J. Schwerdtfeger, A. S. Schneider, C. P.Frick, B. G. Clark, P. A. Gruber and E. Arzt // Philos Mag 88 (2008) 3861.

[243] M. Deutges, I. Knorr, C. Borchers, C. A. Volkert and R. Kirchheim // Scripta Mater 68 (2013) 71.

[244] S. Schreijag, D. Kaufmann, M. Wenk, O. Kraft and R. Monig // Acta Mater 97 (2015) 94.

[245] L. Zhang, T. Ohmura, K. Sekido, T. Hara, K. Nakajima and K. Tsuzaki // Scripta Mater 67 (2012) 388.

[246] Theory of Dislocations 2nd Edition, ed. by J.P. Hirth (John Wiley and Sons: New York, 1982).

[247] G. Taylor // Philos Mag 15 (1967) 873.

[248] B. Sestak // Czech J Phys Sect B B22 (1972) 270.

[249] D. E. Segall, A. Strachan, W. A. Goddard, S. Ismail-Beigi and T. A Arias // Phys Rev $B$ 68 (2003) 014104.

[250] C. S. Roberts, Magnesium and its alloys (Wiley, New York, 1960).

[251] D. H. Bacon, Introduction to Dislocations (Elsevier, Amsterdam, 2011).

[252] M. H. Yoo // Metall Trans A 12 (1981) 409.

[253] D. Hull, Introduction to dislocations (Pergamon Press, Oxford, 1965).

[254] Q. Yu, L. Qi, K. Chen, R. K. Mishra, J. Li and A. M. Minor // Nano Lett 12 (2012) 887.

[255] B. Y. Liu, J. Wang, B. Li, L. Lu, X. Y Zhang, Z. W. Shan, J. Li, C. L. Jia, J. Sun and E. Ma // Nat Commun 5 (2014) 3297. 
[256] H. D. Fan and J. A. El-Awady // J App/ Mech-T Asme 82 (2015) 101006.

[257] K. E. Prasad, K. Rajesh and U. Ramamurty // Acta Mater 65 (2014) 316.

[258] Q. Yu, J. Sun, J. W. Morris and A. M. Minor // Scripta Mater 69 (2013) 57.

[259] X. Z. Tang, Y. F. Guo, S. Xu and Y. S. Wang // Philos Mag 95 (2015) 2013.

[260] Y. F. Guo, X. Z. Tang, Y. S. Wang, Z. D. Wang and S. Yip // Acta Metallurgica Sinica-English Letters 26 (2013) 75.
[261] Y. F. Guo, S. Xu, X. Z. Tang, Y. S. Wang and S. Yip // J Appl Phys 115 (2014) 224902.

[262] J. Q. Ren, Q. Y. Sun, L. Xiao, X. D. Ding and J. Sun // Phil Mag Lett 93 (2013) 583.

[263] G. S. Kim, Small volume investigation of slip and twinning in magnesium single crystals (Université de Grenoble, 2011). 